Eduardo Moscatelli de Souza

Design of Pneumatic and Hydraulic Soft Actuators by Topology Optimization Method 
Eduardo Moscatelli de Souza

\section{Design of Pneumatic and Hydraulic Soft Actuators by Topology Optimization Method}

Submitted in partial fulfillment of the requirements for the degree of Master of Science in Mechanical Engineering 
Eduardo Moscatelli de Souza

\title{
Design of Pneumatic and Hydraulic Soft Actuators by Topology Optimization Method
}

\author{
Revised Version
}

Submitted in partial fulfillment of the requirements for the degree of Master of Science in Mechanical Engineering

Field of Study:

Control and Mechanical Automation Engineering (3152)

Supervised by:

Prof. Dr. Emílio Carlos Nelli Silva 
Autorizo a reprodução e divulgação total ou parcial deste trabalho, por qualquer meio convencional ou eletrônico, para fins de estudo e pesquisa, desde que citada a fonte.

Este exemplar foi revisado e corrigido em relação à versão original, sob responsabilidade única do autor e com a anuência de seu orientador.

São Paulo, de de

Assinatura do autor:

Assinatura do orientador:

Souza, Eduardo Moscatelli de

Design of Pneumatic and Hydraulic Soft Actuators by Topology

Optimization Method / E. M. Souza -- versão corr. -- São Paulo, 2020

$108 p$.

Dissertação (Mestrado) - Escola Politécnica da Universidade de São

Paulo. Departamento de Engenharia Mecatrônica e de Sistemas Mecânicos.

1.Método de Otimização Topológica 2.Método dos Elementos Finitos 3.Mecanismos Flexíveis 4.Robôs Moles 5.Atuadores Moles I.Universidade de São Paulo. Escola Politécnica. Departamento de Engenharia Mecatrônica e de Sistemas Mecânicos II.t. 
Souza, Eduardo Moscatelli de. Design of Pneumatic and Hydraulic Soft Actuators by Topology Optimization Method. 2020. 108 p. Dissertação (Mestrado) - Escola Politécnica, Universidade de São Paulo, São Paulo, 2020.

Aprovado em:

Banca Examinadora

Prof. Dr.

Instituição:

Julgamento:

Prof. Dr.

Instituição:

Julgamento:

Prof. Dr.

Instituição:

Julgamento: 
Dedico esse trabalho aos meus amados pais, Luiza e Valdivino, que sempre me incentivaram, apoiaram e deram suporte aos meus estudos, sendo exemplo de dedicação e empenho.

Também dedico esse trabalho a minha amada esposa, Ariane, que sempre me incentiva, me inspira e me dá forças para perseguir meus sonhos. 


\section{ACKNOWLEDGEMENTS}

Ao meu orientador, Prof. Dr. Emílio Carlos Nelli Silva, pelos conselhos, incentivo, dedicação e paciência enquanto me guiava no desenvolvimento desse trabalho.

Aos meus pais, Luiza e Valdivino, pelo amor, carinho, suporte e dedicação em todos os momentos. Sem vocês eu não teria conseguido concluir esse trabalho. Obrigado por todo sacrifício para me oferecerem a melhor educação possível. Eu precisaria de muitas páginas para agradecer vocês de forma adequada.

À minha esposa, Ariane, pelo amor, pela companhia, pela compreensão, pelos conselhos e pelo incentivo aos meus estudos. Com você minha vida é mais feliz e juntos conseguimos ir mais longe.

Aos meus familiares e amigos, em especial ao meu irmão Marcel e ao meu amigo Sérgio, pelos momentos de descontração que são indispensáveis para recarregar as energias.

À USP e aos professores da Escola Politécnica por toda a infraestrutura para minha formação e realização desse trabalho. 
"We know very little, and yet it is astonishing that we know so much, and still more astonishing that so little knowledge can give us so much power."

Bertrand Russell

"The science of today is the technology of tomorrow."

Edward Teller 


\section{RESUMO}

Robôs moles são máquinas projetadas completamente ou parcialmente a partir de materiais flexíveis (como elastômeros) para contornar limitações presentes em robôs rígidos como dificuldades de operação em ambientes não estruturados e de manuseio de objetos frágeis. Em geral, robôs moles são capazes de realizar movimentos complexos através da deformação de sua própria estrutura. Portanto, é necessário encontrar uma estrutura apropriada para cada requisito de movimento, o que pode ser facilitado por técnicas de automação de projeto. Por isso, recentemente pesquisadores estão investigando técnicas de projeto automático para criar atuadores moles melhores. Neste contexto, esta dissertação é dedicada ao estudo da aplicação do algoritmo de otimização topológica baseado em densidades ao projeto de atuadores moles acionados por carregamentos de pressão (pneumáticos e hidráulicos). A abordagem seguida é sintetizar mecanismos flexíveis atuados por carregamentos dependentes do domínio. Os mecanismos flexíveis são sintetizados através da maximização do deslocamento de saída e o problema de carregamento dependente do domínio é resolvido através do uso de elementos finitos mistos de deslocamento e pressão. Este trabalho mostra que atuadores abertos podem ser obtidos se nenhuma restrição for imposta, porque buracos podem aparecer durante a otimização. Portanto, uma técnica de projeção é proposta para evitar resultados abertos e sua eficácia é demonstrada pela solução de três exemplos: atuador flexor, atuador linear e atuador inversor. O problema de elementos finitos é resolvido com a plataforma FEniCS, a otimização é feita com um algoritmo de ponto interior (IPOPT) e parte da análise de sensibilidades é automatizada com o pacote dolfin-adjoint.

Palavras-chave: Otimização Topológica. Carregamento dependente do domínio. Mecanismos flexíveis. Robôs moles. 


\section{ABSTRACT}

Soft robots are machines completely or partly designed from compliant materials (such as elastomers) to circumvent limitations presented by stiff robots like difficulties to operate in unstructured environments and to handle fragile objects. In general, soft robots are capable of realizing complex movements by the deformation of their own structure. Therefore, it is necessary to find an appropriate structure for each movement requirement. This can be facilitated by automation techniques, so, recently, researchers are investigating automated design techniques to create enhanced soft actuators. In this context, this dissertation is dedicated to study the application of density-based topology optimization to the design of soft actuators driven by pressure loads (pneumatic and hydraulic). The approach followed is to synthesize compliant mechanisms actuated by design dependent loads. The compliant mechanisms are synthesized by selecting the maximization of output displacement as objective function and the design dependent load problem is solved by using mixed displacement-pressure finite elements. This work shows that undesired open designs may be obtained if no constraint is used, because holes may appear during optimization. Therefore, a projection technique is proposed to avoid open designs and its effectiveness is demonstrated by the solution of three numerical examples: a bending, a linear, and an inverter actuator. The finite element problem is solved by using FEniCS framework, the optimization is carried with an interior point algorithm (IPOPT), and part of the sensitivity analysis is automated by dolfin-adjoint package.

Keywords: Topology optimization. Design-dependent loads. Compliant mechanisms. Soft robots. 


\section{LIST OF FIGURES}

Figure 1.1 - Review of soft robots developed in recent years (LASCHI; MAZZOLAI; CIANCHETTI, 2016) . . . . . . . . . . . . . . . . . . . . . 22

Figure 1.2 - Illustration of a soft actuator driven by pressure loads deforming after an internal pressure increase $(\Delta P>0) \ldots \ldots \ldots 22$

Figure 1.3 - Concept of a soft gripper assembled from two soft actuators driven by pressure loads. . . . . . . . . . . . . . . . . . . . . . . . . . 22

Figure 1.4 - Schematic representation of engineering optimization approaches. . . . . . 24

Figure 1.5 - Procedure for the design of a pressure-driven soft actuator by topology optimization. . . . . . . . . . . . . . . . . . . . . . . 24

Figure 2.1 - Kinematic description of the movement of particle $P$ from reference configuration $\Omega_{0}$ to current configuration $\Omega \ldots \ldots \ldots \ldots$. . . . . . . . . . . . . .

Figure 2.2 - Illustration of the relation between pressure $p$ and dilatation $\boldsymbol{\nabla} \cdot \boldsymbol{u}$ in 2D. The REF represents the reference configuration and the DEF represents the deformed configuration (Equation 2.31) . . . . . . . . . . . . . . . . . 34

Figure 2.3 - Threshold projection (Equation 2.58) for different values of $\eta$ and $\beta$. The parameter $\eta$ controls the threshold of projection and the parameter $\beta$ controls the slope of the curve. . . . . . . . . . . . . . . . . . . . . . . 41

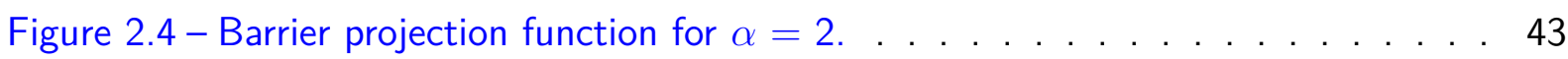

Figure 3.1 - Flowchart describing the algorithm used in this work. . . . . . . . . . . . . 46

Figure 3.2 - Finite element discretization. (a) Displacement discretization; (b) Pressure discretization; (c) Design variable discretization. . . . . . . . . . . . . . . 47

Figure 3.3 - FEniCS' finite element meshes. (a) Right mesh; (b) Left mesh; (c) Crossed mesh. . . . . . . . . . . . . . . . . . . 47

Figure 3.4 - Inverter actuator result obtained with the code presented in Annex D.4. . . 57

Figure 4.1 - Design domain for the problems studied in this work. (a) Bending design domain; (b) Linear and Inverter design domains. . . . . . . . . . . . . . . 58

Figure 4.2 - Design domain with constraints for bending actuator problem. (a) Interior; (b) Interior/Exterior; (c) Barrier. . . . . . . . . . . . . . . . . . . . . . . . 60

Figure 4.3 - Result of interior surfaces optimization for bending actuator problem. (a) Optimized topology; (b) Displacement of optimized topology magnified 10 times; (c) Convergence of objective function and discreteness during optimization. 
Figure 4.4 - Result of interior and exterior surfaces optimization for bending actuator problem. (a) Optimized topology; (b) Displacement of optimized topology magnified 10 times; (c) Convergence of objective function and discreteness during optimization.

Figure 4.5 - Result of barrier optimization for bending actuator problem. (a) Optimized topology; (b) Displacement of optimized topology magnified 10 times; (c) Convergence of objective function and discreteness during optimization. . . 64

Figure 4.6 - Bending actuator results for different values of output port stiffness $\left(k_{\text {out }}\right)$ in $\mathrm{N} / \mathrm{mm}$ (see Table 4.5). . . . . . . . . . . . . . . . 65

Figure 4.7 - Bending actuator optimization using an initial guess with square holes of 15 mm. (a) Initial guess on design variable field; (b) Initial guess projected on physical density field; (c) Result on physical density field. . . . . . . . . . . . 66

Figure 4.8 - Bending actuator optimization using an initial guess with square holes of $25 \mathrm{~mm}$. (a) Initial guess defined on design variable field; (b) Initial guess projected on physical density field; (c) Result on physical density field. . . . 66

Figure 4.9 - Finite deformation of bending actuator for $p_{i n}=7.6 \mathrm{kPa} . \quad \ldots . . .68$

Figure 4.10-Design domain for linear and inverter actuators problems. (a) Interior Surface; (b) Interior/Exterior Surfaces; (c) Barrier Projection. . . . . . . . . . . . . 70

Figure 4.11-Result of interior surfaces optimization for linear actuator problem. (a) Optimized topology; (b) Displacement of optimized topology magnified 250 times; (c) Convergence of objective function and discreteness during optimization.

Figure 4.12-Result of interior and exterior surfaces optimization for linear actuator problem. (a) Optimized topology; (b) Displacement of optimized topology magnified 250 times; (c) Convergence of objective function and discreteness during optimization.

Figure 4.13-Result of barrier optimization for linear actuator problem. (a) Optimized topology; (b) Displacement of optimized topology magnified 250 times; (c) Convergence of objective function and discreteness during optimization. . . 72

Figure 4.14-Finite deformation of linear actuator for $p_{i n}=0.18 \mathrm{MPa} \ldots \ldots$. . . . . 73

Figure 4.15-Result of interior surfaces optimization for inverter actuator problem. (a) Optimized topology; (b) Displacement of optimized topology magnified 250 times; (c) Convergence of objective function and discreteness during optimization.

Figure 4.16-Result of interior and exterior surfaces optimization for inverter actuator problem. (a) Optimized topology; (b) Displacement of optimized topology magnified 250 times; (c) Convergence of objective function and discreteness during optimization. 
Figure 4.17-Result of barrier optimization for inverter actuator problem. (a) Optimized topology; (b) Displacement of optimized topology magnified 250 times; (c) Convergence of objective function and discreteness during optimization. . . 76

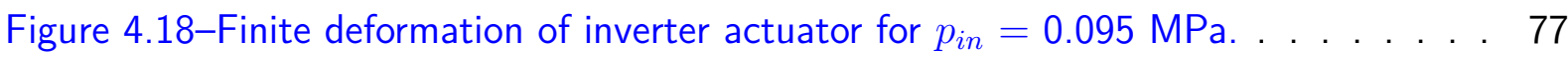

Figure 4.19-Topology optimization results by considering water material properties in fluid phase. (a) Hydraulic bending actuator; (b) Hydraulic linear actuator; (c) Hydraulic inverter actuator. . . . . . . . . . . . . . . . . . . 78

Figure D.1-Design domain of bridge example. The region in black is fixed to be solid. . 95 Figure D.2-Results for bridge example by considering standard displacement and mixed formulation. . . . . . . . . . . . . . . . . . 96

Figure D.3-Bridge topologies by considering different penalization parameters (see Table D.1) . . . . . . . . . . . . . . . . . . . . . 97

Figure D.4-Bridge topologies by considering different penalization parameters and continuation of threshold projection from $\beta_{\min }=1$ to $\beta_{\max }=8$ (see Table D.2) . . . . . . . . . . . . . . . . . . . . . . 98

Figure D.5-Bridge topologies by considering continuation of penalization parameter $z$ (see Table D.3).

Figure D.6-Bridge topologies by considering nearly incompressible material $\nu_{s}=0.49$ (see Table D.4). . . . . . . . . . . . . . . . . . . . . . . . . . . . 99

Figure D.7-Design domain of internally pressurized lid example. . . . . . . . . . . . 100

Figure D.8-Results for internally pressurized lid example by considering different $\nu_{s}$. . . 101

Figure D.9-Results for internally pressurized lid example by considering different $K_{f}$. $\quad 102$

Figure D.10-Results for internally pressurized lid example by considering lower values of $p .102$

Figure D.11-Results for internally pressurized lid example by considering $p=0.01$ and different values of $\epsilon_{t o l} \ldots \ldots \ldots 102$ 


\section{LIST OF TABLES}

Table 4.1 - Material properties used for simulations.

Table 4.2 - Motivation to impose closed constraints or to introduce barrier projection.

The bending actuator problem is solved considering different constraints according to table's header. The initial guess is the closed topology presented in Figure 4.2a for all examples. (a) Column showing results obtained for free optimization; (b) Column showing results obtained for lower edge constraint; (c) Column showing results obtained for closed constraint.

Table 4.3 - Number of finite elements and design variables for bending actuator optimization. 64

Table 4.4 - Bending actuator example summary. . . . . . . . . . . . . . . . . . 65

Table 4.5 - Bending actuator results for different values of output port stiffness $\left(k_{\text {out }}\right)$ in $\mathrm{N} / \mathrm{mm}$ (see Figure 4.6$) \ldots \ldots \ldots 66$

Table 4.6-Summary of density filter parameters $r$ and $l$ effect on barrier projection optimization result. Each row presents results for the same value of $r$ and each column presents results for the same value of $l$. The results marked with a blue dot use the initial guess from Figure 4.7. The result marked with a green dot uses $\epsilon_{t o l}=10^{-3}$. The result marked with a red dot uses $\epsilon_{t o l}=$ $10^{-3}$ and the initial guess of Figure 4.8. The use of different tolerance and initial guesses is made to avoid converge to points of local infeasibility. . . .

Table 4.7 - Finite deformation of bending actuator for different values of input pressure

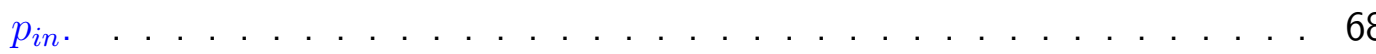

Table 4.8 - Output displacement of bending actuator by considering different FEM models for a input pressure $p_{i n}=0.1 \mathrm{kPa}$. The second, third, and forth rows use the same mesh used for Figure 4.9 . . . . . . . . . . . . . . . 69

Table 4.9 - Number of finite elements and design variables for linear and inverter actuator optimization. . . . . . . . . . . . . . . . . . . . . . 73

Table 4.10-Linear actuator example summary. . . . . . . . . . . . . . . . . . . . . 73

Table 4.11-Finite deformation of linear actuator for different values of input pressure $p_{i n} .73$

Table 4.12-Output displacement of linear actuator by considering different FEM models for a input pressure $p_{i n}=1 \mathrm{kPa}$. The second, third, and forth rows use the same mesh used for Figure 4.14. . . . . . . . . . . . . . . . . . . . . . 74

Table 4.13-Inverter actuator example summary. . . . . . . . . . . . . . . . . . 76

Table 4.14-Finite deformation of inverter actuator for different values of input pressure $p_{i n} .77$

Table 4.15-Output displacement of inverter actuator by considering different FEM models for a input pressure $p_{i n}=0.1 \mathrm{kPa}$. The second, third, and forth rows use the same mesh used for Figure 4.18. . . . . . . . . . . . . . . . . . 77

Table 4.16-Hydraulic actuators example summary. . . . . . . . . . . . . . . . . . . . 78 
Table D.1-Bridge results using different penalization parameters. . . . . . . . . . . . 97

Table D.2-Bridge results by using different penalization parameters and continuation of threshold projection from $\beta_{\min }=1$ to $\beta_{\max }=8 \ldots \ldots 98$

Table D.3-Bridge results by considering continuation of penalization parameter $z$. . . 99

Table D.4-Bridge results by considering nearly incompressible material $\nu_{s}=0.49$. . . 100 


\section{ACRONYMS}

2D Two Dimensional

3D Three Dimensional

FEM Finite Element Method

SIMP Solid Isotropic Material with Penalization

TO Topology Optimization 


\section{NOTATIONS}

\begin{tabular}{|c|c|}
\hline$a$ & Acceleration \\
\hline$b$ & Body forces \\
\hline$C$ & Right Cauchy-Green deformation tensor \\
\hline$C_{F}$ & FEM coupling matrix \\
\hline$D_{F}$ & FEM pressure matrix \\
\hline $\boldsymbol{E}$ & Green-Lagrange strain tensor \\
\hline$E_{Y}$ & General Young's modulus \\
\hline$E_{S}$ & Young's modulus of solid regions \\
\hline$E_{V}$ & Young's modulus of void regions \\
\hline$f_{F}$ & FEM load vector \\
\hline $\boldsymbol{F}$ & Deformation gradient \\
\hline$G$ & Shear modulus \\
\hline $\boldsymbol{I}$ & Identity matrix \\
\hline$K$ & Bulk modulus \\
\hline $\boldsymbol{K}_{\boldsymbol{F}}$ & FEM stiffness matrix \\
\hline$n_{\max }$ & Maximum number of iterations of optimization \\
\hline$n$ & Normal vector \\
\hline$N_{p}$ & Vector of pressure shape functions \\
\hline$N_{u}$ & Matrix of displacement shape functions \\
\hline$N_{\rho}$ & Vector of density shape functions \\
\hline$p$ & Pressure \\
\hline$\tilde{\boldsymbol{p}}$ & Global vector of pressure degrees of freedom \\
\hline$p_{e}$ & Pressure on a finite element \\
\hline$\hat{p}_{e}$ & Pressure approximation on a finite element \\
\hline$\tilde{p}_{e}$ & Vector of pressure degrees of freedom on a finite element \\
\hline$\delta p$ & Pressure test function \\
\hline$S$ & Second Piola-Kirchhoff strain tensor \\
\hline$t$ & Time \\
\hline$t$ & Surface forces \\
\hline$u$ & Displacement \\
\hline$\tilde{\boldsymbol{u}}$ & Global vector of displacement degrees of freedom \\
\hline$u_{e}$ & Displacement on a finite element \\
\hline$\hat{\boldsymbol{u}}_{e}$ & Displacement approximation on a finite element \\
\hline$\tilde{\boldsymbol{u}}_{\boldsymbol{e}}$ & Vector of displacement degrees of freedom on a finite element \\
\hline$\delta u$ & Displacement test function \\
\hline
\end{tabular}




$\begin{array}{ll}\boldsymbol{v} & \text { Velocity } \\ W & \text { Strain energy density function } \\ X & \text { Reference configuration (material coordinates) } \\ x & \text { Current configuration (spatial coordinates) } \\ z & \text { SIMP penalization parameter } \\ \epsilon & \text { Desired convergence tolerance of optimization } \\ \boldsymbol{\epsilon} & \text { Infinitesimal strain tensor } \\ \boldsymbol{\epsilon}_{V} & \text { Infinitesimal strain tensor in Voigt notation } \\ \boldsymbol{\epsilon}^{\prime} & \text { Deviatoric strain tensor } \\ \lambda & \text { Lamé's first parameter } \\ \mu & \text { Lamé's second parameter } \\ \nu & \text { Poisson's ratio } \\ \rho & \text { Design variable } \\ \bar{\rho} & \text { Density-filtered variable (traditional formulation) or smoothed } \\ \hat{\rho} & \text { variable (barrier projection formulation) } \\ \tilde{\rho} & \text { Threshold projected variable } \\ \overline{\bar{\rho}} & \text { Barrier projected variable } \\ \boldsymbol{\sigma} & \text { Density-filtered variable in barrier projection } \\ \boldsymbol{\sigma} & \text { Cauchy stress tensor } \\ \Omega & \text { Cauchy stress tensor in Voigt notation } \\ \partial \Omega & \text { Domain } \\ & \text { Boundary of the domain }\end{array}$




\section{CONTENTS}

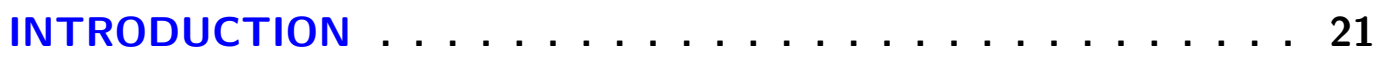

1.1 Soft Robots, Soft Actuators, and Soft Sensors . . . . . . . . . . 21

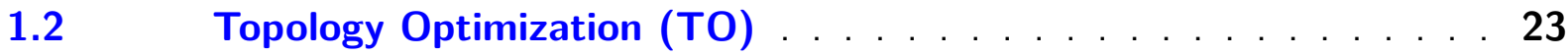

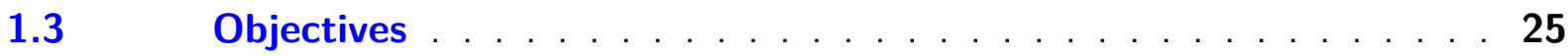

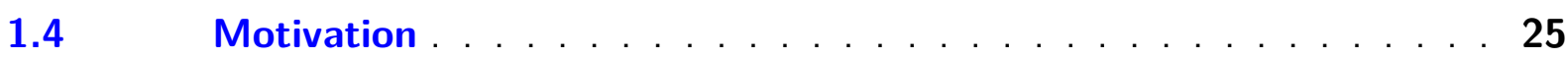

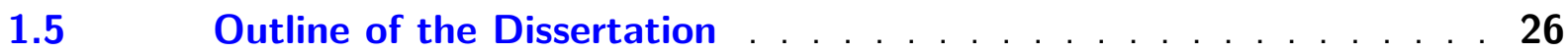

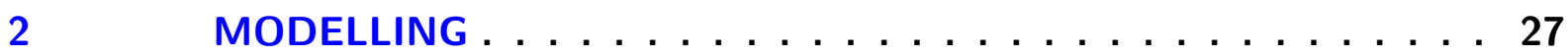

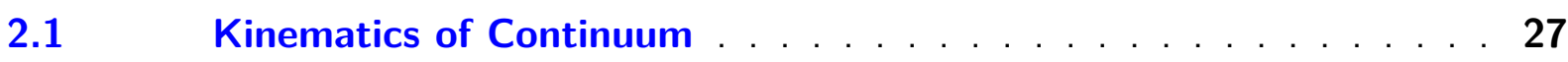

2.2 Deformation and Strain . . . . . . . . . . . . . . . 29

2.3 Dilatation . . . . . . . . . . . . . . . . . 30

$2.4 \quad$ Cauchy Stress Tensor . . . . . . . . . . . . . . 30

$2.5 \quad$ Equations of Motion: Principle of Linear Momentum . . . . . . . 30

2.6 Symmetry of Stress Tensor: Principle of Angular Momentum . . . 31

$2.7 \quad$ Motion Problem . . . . . . . . . . . . . . . . . 31

$2.8 \quad$ Isotropic Linearly Elastic Solid . . . . . . . . . . . . . . 32

$2.9 \quad$ Incompressibility . . . . . . . . . . . . . . . . . 33

$2.10 \quad$ Mixed u-p formulation . . . . . . . . . . . . . . . . . 34

2.11 Finite Element Method . . . . . . . . . . . . . . . 35

$2.11 .1 \quad$ Mixed displacement-pressure equations . . . . . . . . . . . . . 35

2.12 Topology Optimization Method . . . . . . . . . . . . . . 38

2.12 .1 Continuation . . . . . . . . . . . . . . . . . . . 42

$2.12 .2 \quad$ Barrier Projection . . . . . . . . . . . . . . . . . . . . 42

$2.12 .3 \quad$ Compliant Mechanisms . . . . . . . . . . . . . . . . . . . 44

2.12 .4 Design Dependent Loads . . . . . . . . . . . . . . . . . . . 45

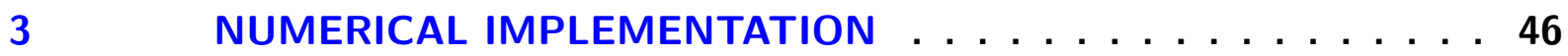

$3.1 \quad$ Algorithm . . . . . . . . . . . . . . . . . . 46

3.2 Sensitivity Analysis . . . . . . . . . . . . . . 48

$3.3 \quad$ Inverter Mechanism Design in FeniCS . . . . . . . . . . 51

$4 \quad$ RESULTS . . . . . . . . . . . . . . . 58

$4.1 \quad$ Bending Actuator . . . . . . . . . . . . . . . . 59

$4.1 .1 \quad$ Motivation . . . . . . . . . . . . . . . . 61

$4.1 .2 \quad$ Interior Surface . . . . . . . . . . . . . . . . . . 62

$4.1 .3 \quad$ Interior $/$ Exterior Surfaces . . . . . . . . . . . . . . . 63 
4.1.4 Barrier Projection . . . . . . . . . . . . . . . . 63

4.1.5 Comparison of Results . . . . . . . . . . . . . . . . . . . . . . 64

4.1.6 Effect of Output Port Stiffness . . . . . . . . . . . . . . . . . 65

$4.1 .7 \quad$ Initial Guess Effect . . . . . . . . . . . . . . . . . . . 65

4.1.8 Effect of Filter Parameters $r$ and I . . . . . . . . . . . . . . . 67

$4.1 .9 \quad$ Finite Deformation . . . . . . . . . . . . . . . . . 67

$4.2 \quad$ Linear Actuator . . . . . . . . . . . . . . . . . . . . . . . 69

$4.2 .1 \quad$ Interior Surface . . . . . . . . . . . . . . . . . . . . 70

4.2.2 Interior $/$ Exterior Surfaces . . . . . . . . . . . . . . . . . 71

$4.2 .3 \quad$ Barrier Projection . . . . . . . . . . . . . . . . . . . . . . 71

4.2.4 Comparison of Results . . . . . . . . . . . . . . . . . . 71

$4.2 .5 \quad$ Finite Deformation . . . . . . . . . . . . . . . . . 73

$4.3 \quad$ Inverter Actuator . . . . . . . . . . . . . . . . . . . . . . . . . 74

4.3.1 Interior Surface . . . . . . . . . . . . . . . . . . . . . . . . . . . . . . . . 74

$4.3 .2 \quad$ Interior $/$ Exterior Surfaces . . . . . . . . . . . . . . . . . . 74

4.3.3 Barrier Projection . . . . . . . . . . . . . . . . . . . . . 75

$4.3 .4 \quad$ Comparison of Results . . . . . . . . . . . . . . . . . . 76

4.3.5 Finite Deformation . . . . . . . . . . . . . . 76

$4.4 \quad$ Hydraulic Actuators . . . . . . . . . . . . . . . . . . . . 77

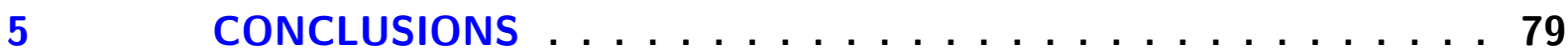

$5.1 \quad$ Future Work . . . . . . . . . . . . . . . . . . . 80

REFERENCES ...................... . . 81

APPENDIX A - RELATION BETWEEN $K, E_{Y}$ AND $\nu \ldots . . .86$

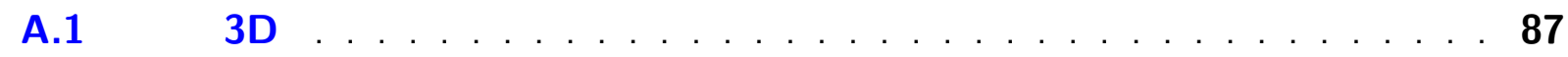

A.2 $\quad$ 2D Plane Stress $\ldots \ldots \ldots \ldots \ldots \ldots$

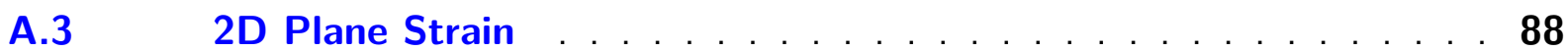

APPENDIX B - NONLINEAR MATERIAL MODEL . . . . . . 89

B.1 Hyperelastic Material . . . . . . . . . . . . . . . 89

B.1.1 Neo-Hookean Solid . . . . . . . . . . . . . . . . . . . . . . . . . . 90

B.2 Newton's Method . . . . . . . . . . . . . . . . . . . . 91

APPENDIX C - DERIVATIVES OF STRAIN ENERGY DENSITY FUNCTIONS ................ 93

APPENDIX D - IMPLEMENTATION IN FENICS . . . . . . . 94

D.1 Bridge example . . . . . . . . . . . . . . . . . . . . . . 94

D.1.1 Comparison to standard displacement formulation . . . . . . . . . . . 96 


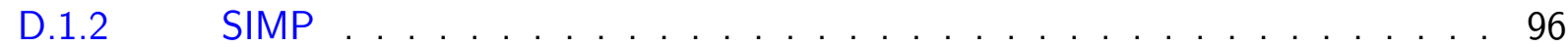

D.1.3 SIMP with threshold projection continuation . . . . . . . . . . . . 97

D.1.4 SIMP continuation with threshold projection continuation . . . . . . . . 98

D.1.5 Nearly incompressibility . . . . . . . . . . . . . . . . . . . . . . 99

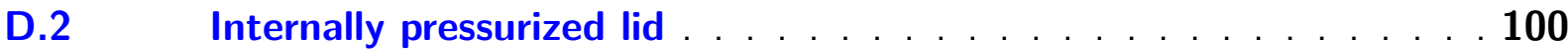

D.2.1 Fluid compressibility . . . . . . . . . . . . . . . . . . 101

D.2.2 Pressure load magnitude . . . . . . . . . . . . . . . . 101

D.3 Air Bulk Modulus . . . . . . . . . . . . . . . . . . . . 103

D.4 Complete Code of Inverter Actuator Design . . . . . . . . . . . . . 103 


\section{INTRODUCTION}

This dissertation is dedicated to the application of the Topology Optimization (TO) method to the design of pneumatic and hydraulic soft actuators. This section introduces the use of soft components in robot design and the state of art of TO. It also presents the objectives and motivation of this work as well as its outline.

\subsection{Soft Robots, Soft Actuators, and Soft Sensors}

Robots are machines capable of sensing its surrounding environment and acting on it according to computer programs embedded on them. Traditionally, they are employed in industry to perform repetitive tasks such as soldering mechanical components and moving objects. Industrial environments are called structured environments because the operation conditions are known or can be measured. However, in recent years the application of robots has expanded to our daily life and it is possible to find robots vacuuming houses, monitoring electrical grids and farms. Also, there is great interest of using robots in healthcare (e.g. da Vinci Surgical System), education, retail stores, harvesting, and others. These new fields of robot application present unknown ambient conditions, because the conditions are not fully controllable, or can change over time, or can not be measured, among other reasons. Therefore, they are considered unstructured environments.

One great difficult to design robots that operate in unstructured environments or that handle fragile objects is that traditional robots rely on precision. If a rigid robot makes a positioning error, it can damage its work-piece or its own structure. This is a prohibitive characteristic for applications such as human interaction and handling fragile objects.

One way to overcome this problem is to use soft materials such as rubber and fabric to design mechanical components, actuators and sensors that can be applied to robots. This subject has been studied in last decades under the names of soft robots, soft actuators, and soft sensors. Figure 1.1 presents several soft robots developed in recent years following different principles and ideas.

One technique that has been explored to provide motion to soft robots is pneumatic/hydraulic actuation. Figure 1.2 illustrates a soft actuator driven by pressure loads at rest $(\Delta P=0)$ and during the start of its deformation $(\Delta P>0)$. This actuator can be used in the assemble of more complex components such as the schematic soft gripper presented in Figure 1.3. This idea can be used, for example, to design rehabilitation gloves such as the one presented in Figure 1.1.

One of the pioneers works in soft robots is presented by Suzumori (1996). The author 


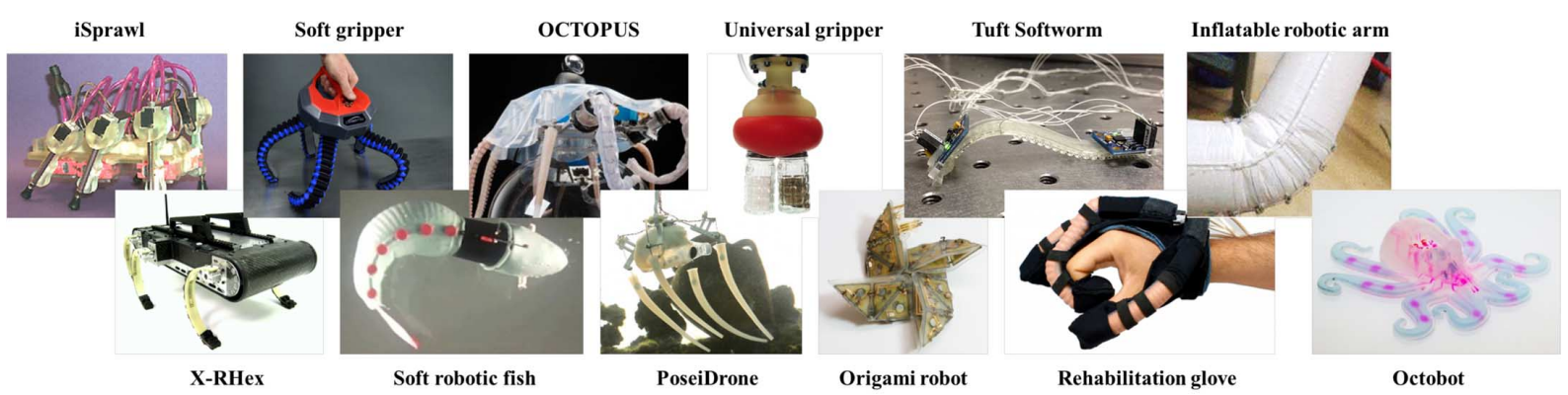

Figure 1.1 - Review of soft robots developed in recent years (LASCHI; MAZZOLAI; CIANCHETTI, 2016)
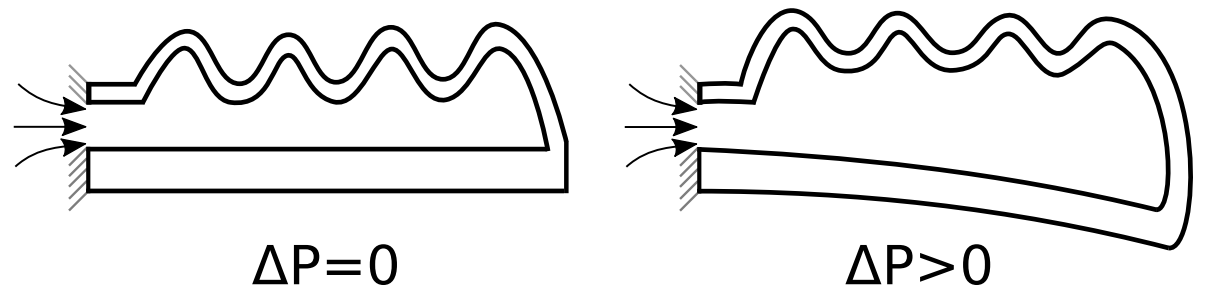

Figure 1.2 - Illustration of a soft actuator driven by pressure loads deforming after an internal pressure increase $(\Delta P>0)$.

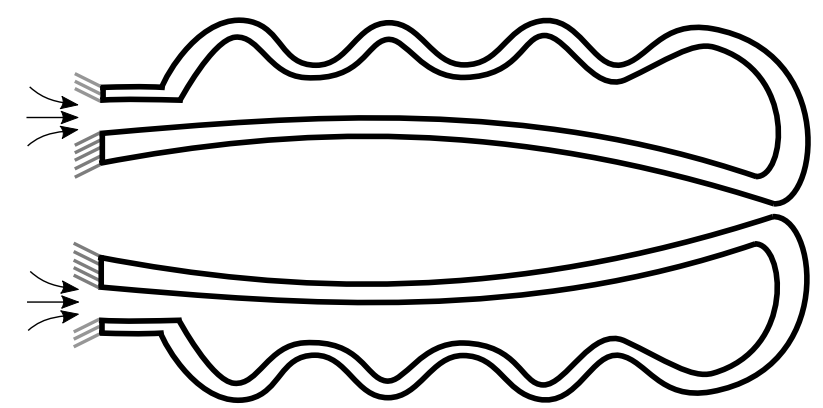

Figure 1.3 - Concept of a soft gripper assembled from two soft actuators driven by pressure loads.

designed a pneumatic actuator fabricated from silicone rubber reinforced with nylon fiber. The actuator is embedded with three chambers and it is able to bend in any direction by the appropriate pressurization of one of its chambers. The author uses this actuator to create a robot arm, a multi-fingered robot hand, and walking robots showing thus the potential of compliant materials in robot design.

Galloway et al. (2013) devise an empirical method for adjusting the bending radius of fiber-reinforced soft actuators. The authors use sleeves (conformal coverings) to constraint the motion of parts of the actuator. The developed method allows the end user to mechanically program the bending radius of the actuator according to the application.

In Mosadegh et al. (2014), the authors present pneumatic networks that require less gas for actuation. This improvement reduces the actuation time of the actuators as well as the fatigue and failure, because less volume change implies in less strain. 
Gong et al. (2016) present soft rotary actuators that convert peristaltic motion in torque. The peristaltic motion is obtained by the sequential inflation of groups of bladders embedded on the stator of the actuator. Two configuration of actuators are presented: the first type has an inflatable stator surrounding an internal rotor while the second type of actuator has an external rotor surrounding the stator. The authors demonstrate the application of the actuators in a four-wheeled vehicle capable of moving in rocky terrain with a puddle in its path and capable of sustaining a fall from eight times its height.

Researchers have also shown interest in designing sensors from soft materials for sophisticated applications in soft robots. For example, Park et al. (2010) present the design of an elastomeric sensor embedded with channels filled with liquid eutectic gallium-indium (eGaln). When the surfaces of the sensor are pressurized, the cross section area of the channels and, therefore, the electrical resistance change. The relation between change in electrical resistance and pressure is obtained by a closed-form equation derived from plane strain elasticity, contact mechanics and linear elastic fracture mechanics (LEFM). The designed sensor is able to measure pressures in the range of $0-100 \mathrm{kPa}$ with $1 \mathrm{kPa}$ of resolution.

In So et al. (2009), the authors present deformable dipole antennas fabricated from elastomeric materials embedded with microchannels of fluid metal alloy (EGaln). The antennas can be stretched, bent, rolled, and twisted reversibly without any hysteresis or loss of electrical continuity.

Zhao et al. (2016) explore the optical properties of materials to design soft sensors. They designed stretchable waveguides that are capable of measuring deformation by the light loss during the propagation through the channel. The authors embedded the proposed sensors in a prosthetic pneumatic hand being able to measure shape and texture of surfaces by touching them.

Rothemund et al. (2018) present a soft pneumatic valve that can replace hard valves in soft robots design. The valve presents a bistable membrane capable of controlling the air flow by using the "snap-through" instability. The authors demonstrate the potential of the valve by showing a pneumatic switch and an oscillator created by using the soft valve as functional element. Also, the authors apply the soft valve to a soft gripper and to a soft earthworm robot.

The interest of this dissertation is to study the design automation of actuators driven by pressure loads such as pneumatic and hydraulic soft actuators. To this end, TO has been chosen as the optimization technique.

\subsection{Topology Optimization (TO)}

The optimization of an engineering component consists of maximizing its performance given some constraints. The approaches to carry out this task are grouped according to the 


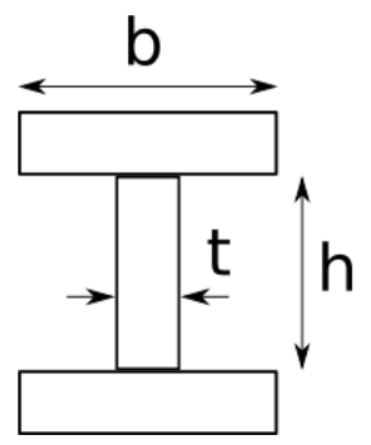

Parametric

Optimization

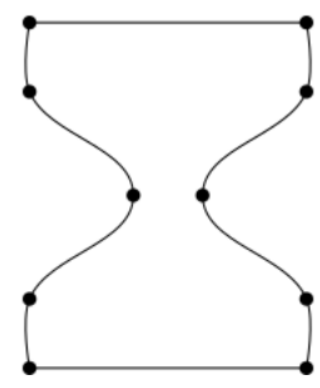

Shape

Optimization

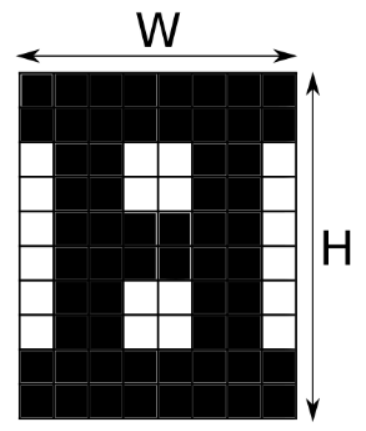

Topology Optimization

Figure 1.4 - Schematic representation of engineering optimization approaches.
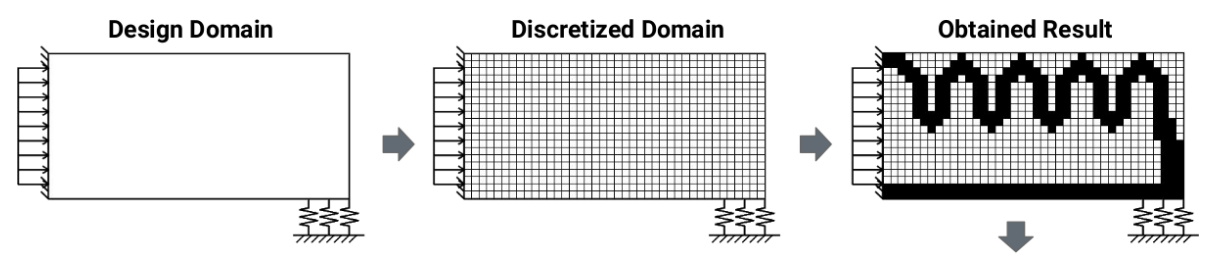

Fabrication

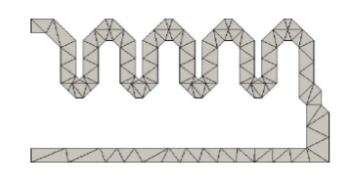

Verification

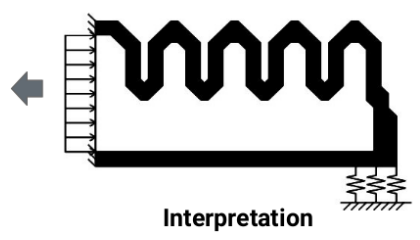

Figure 1.5 - Procedure for the design of a pressure-driven soft actuator by topology optimization.

possibilities that they present.

In order of generality, the first approach is parametric optimization which consists of defining parameters (e.g., the length and height of the rectangular cross section of a beam) of the component as design variables (i.e., values that the optimization algorithm can vary during its execution). The second approach is shape optimization which consists of defining the components by parametric shapes whose coefficients are design variables. Finally, topology optimization consists of dividing the design domain in pixels (for 2D problems) or voxels (for $3 \mathrm{D}$ problems) and assigning to each of these elements a design variable that indicates the presence or absence of material. An schematic representation of these three approaches is presented in Figure 1.4.

Topology optimization is capable of introducing new features (e.g. holes) to the design without prescriptions. This makes the method more general than parametric and shape optimization leveraging the performance of the obtained component. Therefore, it is the method used in this dissertation. The steps of topology optimization are presented in Figure 1.5 for the design of a pressure-driven soft actuator.

The interest of applying topology optimization to the design of soft robots and sensors is 
already manifested in literature. Hiller and Lipson (2012) employ evolutionary algorithms to automate the design of freeform soft robots capable of forward locomotion when volumetrically expanded. The design technique is an evolutionary topology optimization method based on level-set concept and Gaussian mixtures. The authors also fabricated the freeform robots from foam rubbers in a semi automatic process using a laser cutter.

Chen et al. (2018) project a soft gripper driven by cables using the level set topology optimization method. The authors varied the position and the area of contact forces to check its influence in optimized shapes.

Deng, Cheng and To (2018) studied the failure of hyperelastic materials with a finite strain topology optimization algorithm. The authors propose a new objective function to avoid concentration of distortion energy. This approach reduces problems such as stress softening caused by repeated cycles of large deformations.

Zhang et al. (2018) present the design and fabrication of a pneumatic gripper from the assemble of two topology-optimized soft actuators. The authors solve a compliant mechanism optimization problem (maximization of output displacement) to optimize the output surface of a hollow cuboid. The authors do not consider design-dependent loads in their work as a thin layer of material is fixed in the interior of the actuator during optimization.

\subsection{Objectives}

The objective of this work is to explore the application of TO to the design of pneumatic and hydraulic soft actuators, which comprises to simulate numerically these actuators and to execute optimization routines of its material distribution. The simulations and optimizations consider linear material behavior and linear displacements/strains as a first approach to the problem.

The density-based topology optimization method is applied to the design of pressure-loaded compliant mechanisms by using mixed displacement-pressure finite elements (SIGMUND; CLAUSEN, 2007). It is verified that open designs are obtained when running the optimization by using just traditional density filtering (LAZAROV; SIGMUND, 2010) and threshold projection techniques (WANG; LAZAROV; SIGMUND, 2010). Therefore, the optimization is first executed by setting fixed enclosures. Then, a projection technique is proposed to enable the simultaneous optimization of interior and exterior surfaces of the actuator.

\subsection{Motivation}

In general, soft actuators' design is based on knowledge, experience, and intuition of designers who choose the geometry that guarantees that actuators will execute the desired 
motion. However, as these devices realize prescribed motion, designers needs to do a new project when force or displacement requirements change. So the automation and customization of designs are of great interest in literature as can been in works such as Hiller and Lipson (2012) and Galloway et al. (2013).

In this context, TO is a design automation technique with potential to leverage the design of soft actuators because its procedure reproduces the steps taken by designers to devise new actuators. TO seeks a material distribution that meets some design objective that can be translated with an appropriate formulation to find a geometry that produces the desired motion. The success of this task allows the generation of new actuators by the change of parametric input to the automation software, so that the designer efforts need to be employed only in the evaluation of the obtained topology.

Additionally, in the future, TO can enhance the performance of soft actuators by the inclusion of new design objectives such as the reduction of actuation time and the reduction of weight. As presented by Mosadegh et al. (2014), the actuation time is reduced when the amount of air required to drive the device is also reduced. In topology optimization, this could be handled using the volume constraint, for example, by limiting the fluid volume. Another alternative would be to find the shortest trajectory. The weight reduction would also be handled by the volume constraint, but by limiting the solid volume.

These possibilities are enough to justify the study of TO as a design tool for soft actuators, but they are just a few ideas from the synergy of these fields. In this work, we focus our attention on pneumatic and hydraulic actuators because, according to Trimmer (2017), pneumatic devices are the devices most studied until now and hydraulic actuators can be designed with similar formulations by adjusting material parameters.

\subsection{Outline of the Dissertation}

The remainder of this dissertation is organized as follows: in Chapter 2, the equations and assumptions used during actuators modelling are presented. The solid equations are obtained from continuum mechanics principles and discretized by the Finite Element Method (FEM). Then, the Topology Optimization formulation applied in this work is developed. In Chapter 3 , the algorithm and sensitivity analysis used in this work are presented. Also, the software packages used are listed and described. In Chapter 4, the results obtained for the optimization of the actuators are presented. In Chapter 5, the dissertation ends with the conclusions drawn from the work. 


\section{MODELLING}

In this chapter, the physical modelling of pressure loaded structures is presented. First, kinematic concepts of continuum mechanics are explored. Then, the generalized equation of motion for continuum structures is presented and applied to isotropic linearly elastic solid (LAl; RUBIN; KREMPL, 2009; MASE; MASE, 1999). The final equations are discretized by Finite Element Method to permit its numerical solution.

The chapter proceeds developing the Topology Optimization formulations used in this work and the proposed Barrier Projection technique which is published in Souza and Silva (2020).

\subsection{Kinematics of Continuum}

The description of motion is made considering the abstraction of a continuum which implies that the body fully occupies space. This assumption is precise in this work because the length scales considered are much greater than inter-atomic distances.

The approach to model the kinematics of a continuum is to consider the movement of a body particle $P$ from a reference configuration $\Omega_{0} \subset \mathbb{R}^{3}$ to the current configuration $\Omega \subset \mathbb{R}^{3}$. In the reference configuration, the particle position is represented by the reference coordinate $X \in \Omega_{0}$. As the body moves, the particle position changes to the current coordinate $x \in \Omega$. This is illustrated in Figure 2.1.

The definition of coordinates $X$ and $x$ permits two approaches to write down equations for physical laws. The first approach is to consider the evolution of a physical quantity $\Theta$ following a particle of the body. This can be achieved considering the quantity as a function of the reference coordinates $\Theta(X)$. For this reason the reference coordinates are also knows as material coordinates. This approach is known as material description or Lagrangean description.

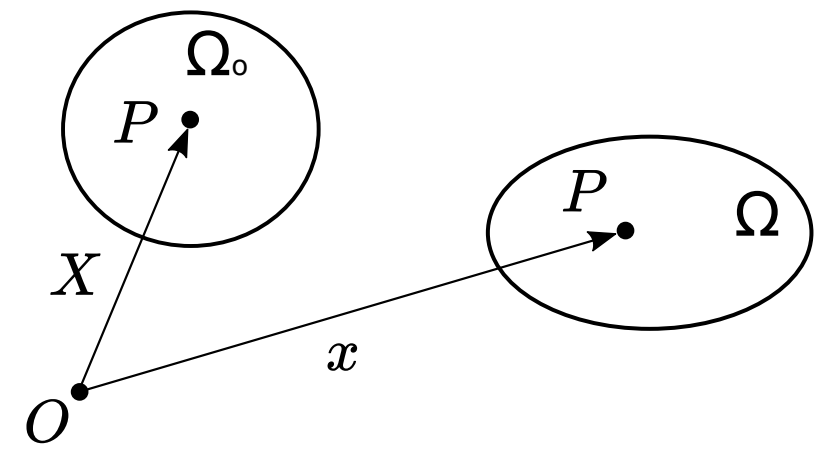

Figure 2.1 - Kinematic description of the movement of particle $P$ from reference configuration $\Omega_{0}$ to current configuration $\Omega$. 
The second approach is to consider how a physical quantity changes at a specific point of space. This can be achieved by writing equations for the particles that pass through the point of coordinates $x$ at time $t$. This approach is known as spatial description or Eulerian description.

The Lagrangean description is commonly employed for solids and the Eulerian description is the most employed for fluid simulations.

The displacement vector measures the difference between the current configuration $\boldsymbol{x}$ and the reference configuration $\boldsymbol{X}$ according to Equation 2.1.

$$
\boldsymbol{u}(\boldsymbol{X}, t)=\boldsymbol{x}(\boldsymbol{X}, t)-\boldsymbol{X}
$$

The velocity $\boldsymbol{v}$ and acceleration $\boldsymbol{a}$ of a particle are obtained by differentiating Equation 2.1 with respect to time according to Equations 2.2 and 2.3.

$$
\begin{gathered}
\boldsymbol{v}=\frac{d \boldsymbol{u}}{d t} \\
\boldsymbol{a}=\frac{d^{2} \boldsymbol{u}}{d t^{2}}
\end{gathered}
$$

By considering two arbitrary points $\mathrm{P}$ and $\mathrm{Q}$ of a continuum, the vector connecting the points $\boldsymbol{d} \boldsymbol{X}$ is transformed in $\boldsymbol{d} \boldsymbol{x}$ after motion. The tensor $\boldsymbol{F}$ that describes this transformation is called transformation gradient and it is defined by Equation 2.4.

$$
d x=F d X=\frac{\partial x}{\partial X} d X=\nabla_{X} d X
$$

The operator $\nabla_{\boldsymbol{X}}$ is the gradient with respect to reference coordinates $\boldsymbol{X}$. The gradient with respect to current coordinates $\boldsymbol{x}$ is the operator $\boldsymbol{\nabla}_{\boldsymbol{x}}$ that is obtained by inverting Equation 2.4 according to Equation 2.5.

$$
d X=F^{-1} d x=\frac{\partial X}{\partial x} d x=\nabla_{x} d x
$$

By differentiating Equation 2.1 with respect to $\boldsymbol{X}$, a new expression for $\boldsymbol{F}$ is obtained according to Equation 2.6 where $\boldsymbol{I}$ is the identity matrix.

$$
\boldsymbol{F}=\boldsymbol{I}+\nabla_{\boldsymbol{X}} \boldsymbol{u}
$$

The deformation gradient $\boldsymbol{F}$ plays a central hole in continuum mechanics and in finite 
deformation formulations and it is used to change the equations from Lagrangian to Eulerian description.

During pure translation, the vectors $\boldsymbol{d} \boldsymbol{X}$ and $\boldsymbol{d} \boldsymbol{x}$ are equal $\boldsymbol{F}=\boldsymbol{I}$, so the deformation gradient contains no information about the body translation. Also, if $\boldsymbol{F}=\boldsymbol{I}$ in all particles of the body, there is no deformation or translation.

\subsection{Deformation and Strain}

The deformation is the relative displacement between the particles of a continuum, i.e., the deformation is the difference between the distance of the particles in reference and current configurations. Therefore, the deformation has units of length. The strain is the ratio of deformation to initial length and it is unitless.

The deformation can be measured by considering again two particles $\mathrm{P}$ and $\mathrm{Q}$ separated by vector $\boldsymbol{d} \boldsymbol{X}$ in reference configuration and by $\boldsymbol{d} \boldsymbol{x}$ in current configuration. The distance between these particles in reference configuration $d S$ and in current configuration $d s$ are given by Equation 2.7 where $C$ is the right Cauchy-Green deformation tensor.

$$
\begin{aligned}
(d S)^{2} & =\boldsymbol{d} \boldsymbol{X} \cdot \boldsymbol{d} \boldsymbol{X} \\
(d s)^{2} & =\boldsymbol{d} \boldsymbol{x} \cdot \boldsymbol{d} \boldsymbol{x}=(\boldsymbol{F} \boldsymbol{d} \boldsymbol{X}) \cdot(\boldsymbol{F} \boldsymbol{d} \boldsymbol{X})=\left(\boldsymbol{d} \boldsymbol{X}^{T} \boldsymbol{F}^{T}\right)(\boldsymbol{F} \boldsymbol{d} \boldsymbol{X}) \\
& =\boldsymbol{d} \boldsymbol{X} \cdot\left(\boldsymbol{F}^{T} \boldsymbol{F}\right) \boldsymbol{d} \boldsymbol{X}=\boldsymbol{d} \boldsymbol{X} \cdot \boldsymbol{C} \boldsymbol{d} \boldsymbol{X}
\end{aligned}
$$

Therefore, the difference between the squares of the distances $d s$ and $d S$ can be written in relation to the reference configuration according to Equation 2.8 where $\boldsymbol{E}$ is the Green-Lagrange strain tensor.

$$
\begin{aligned}
(d s)^{2}-(d S)^{2} & =(\boldsymbol{d} \boldsymbol{X} \cdot \boldsymbol{C} \boldsymbol{d} \boldsymbol{X})-(\boldsymbol{d} \boldsymbol{X} \cdot \boldsymbol{d} \boldsymbol{X}) \\
& =\boldsymbol{d} \boldsymbol{X} \cdot(\boldsymbol{C}-\boldsymbol{I}) \boldsymbol{d} \boldsymbol{X}=\boldsymbol{d} \boldsymbol{X} \cdot 2 \boldsymbol{E} \boldsymbol{d} \boldsymbol{X}
\end{aligned}
$$

The Green-Lagrange strain tensor $\boldsymbol{E}$ can be expressed as a function of the displacement $\boldsymbol{u}$ of the particle according to Equation 2.9.

$$
\boldsymbol{E}(\boldsymbol{u})=\frac{1}{2}\left(\nabla \boldsymbol{u}+\nabla \boldsymbol{u}^{T}+\nabla \boldsymbol{u}^{T} \nabla \boldsymbol{u}\right)
$$

When the components of displacement vector $\boldsymbol{u}$ or its partial derivatives are small, the Green-Lagrange strain tensor $\boldsymbol{E}$ can be approximated to the infinitesimal strain tensor $\boldsymbol{\epsilon}(\boldsymbol{u})$ 
which is given by Equation 2.10.

$$
\boldsymbol{\epsilon}(\boldsymbol{u})=\frac{1}{2}\left(\nabla \boldsymbol{u}+\nabla \boldsymbol{u}^{T}\right)
$$

\subsection{Dilatation}

The dilatation $(e)$ is the change of volume $(\Delta V)$ per unit of initial volume $\left(V_{0}\right)$. It is defined by Equation 2.11.

$$
e=\frac{\Delta V}{V_{0}}=\operatorname{tr}(\boldsymbol{\epsilon})=\nabla \cdot \boldsymbol{u}
$$

As the dilatation $e$ represents a change in volume, it is independent of the direction of the particle considered. Therefore, $e$ is an invariant of the strain tensor $\boldsymbol{\epsilon}$.

\subsection{Cauchy Stress Tensor}

Stress is the physical quantity that describes the magnitude and direction of forces applied on a particle by adjacent particles (internal forces) of a continuum. As the forces applied by adjacent particles varies with direction, the stress vector on a given point in space varies according to the considered plane. To describe the stress state for a particle with respect to all planes that pass through the particle, the Cauchy stress tensor (or simply stress tensor) $\sigma$ is defined. The stress vector $\boldsymbol{\sigma}_{n}$ in a plane whose normal direction is given by vector $\boldsymbol{n}$ is given Equation 2.12.

$$
\sigma_{n}=\sigma n
$$

\subsection{Equations of Motion: Principle of Linear Momentum}

The differential equations of motion for a continuum are obtained by applying Newton's second law of motion to every particle of the continuum $\Omega$. By considering a body subjected to volume loads $\boldsymbol{b}$ (e.g. weight and magnetic forces), the equilibrium of forces is given by Equation 2.13.

$$
\rho \boldsymbol{a}=\boldsymbol{b}+\nabla \cdot \boldsymbol{\sigma} \text { in } \Omega
$$

Equation 2.13 is known as Cauchy's equation of motion. This equation holds for a particle that is currently at position $\boldsymbol{x}$. However, when the displacements $\boldsymbol{u}$ associated with the motion 
are small (i.e. $\boldsymbol{x} \approx \boldsymbol{X}$ ), there is no need to make distinction between spatial and material coordinates.

The boundary condition are obtained by matching surface loads $t$ and stress in normal direction over the body surface $\partial \Omega$ according to Equation 2.12 .

\subsection{Symmetry of Stress Tensor: Principle of Angular Momentum}

The application of the Principle of Angular Momentum shows the symmetry of the stress tensor (Equation 2.14). This relation is important when deriving the integral equations of motion.

$$
\boldsymbol{\sigma}=\boldsymbol{\sigma}^{T}
$$

\subsection{Motion Problem}

The motion problem is formulated by considering the equations of motion (Equation 2.13) and boundary conditions as a system of equations. In differential form, the problem is written as Equation 2.15.

$$
\begin{array}{lr}
\rho \boldsymbol{a}=\boldsymbol{b}+\nabla \cdot \boldsymbol{\sigma} & \text { in } \Omega \\
\boldsymbol{\sigma} \boldsymbol{n}=\boldsymbol{t} & \text { on } \partial \Omega
\end{array}
$$

If the problem is static, the acceleration is zero $(\boldsymbol{a}=\mathbf{0})$ and the problem is reduced to Equation 2.16.

$$
\begin{array}{lr}
\boldsymbol{b}+\nabla \cdot \boldsymbol{\sigma}=\mathbf{0} & \text { in } \Omega \\
\boldsymbol{\sigma} \boldsymbol{n}=\boldsymbol{t} & \text { on } \partial \Omega
\end{array}
$$

The integral formulation of this problem is obtained by multiplying both equations by a test function $\delta \boldsymbol{u}$, by integrating over the valid domain and applying Green's theorem according to Equations 2.17, 2.18, and 2.19. The symmetry of stress tensor is used in manipulations made in Equation 2.18.

$$
\begin{gathered}
\int_{\Omega} \boldsymbol{b} \cdot \boldsymbol{\delta} \boldsymbol{u}+(\nabla \cdot \boldsymbol{\sigma}) \cdot \boldsymbol{\delta} \boldsymbol{u} d \Omega=0 \\
\int_{\Gamma} \boldsymbol{t} \cdot \boldsymbol{\delta} \boldsymbol{u} d \Gamma=\int_{\Gamma}(\boldsymbol{\sigma} \boldsymbol{n}) \cdot \boldsymbol{\delta} \boldsymbol{u} d \Gamma=\int_{\Gamma} \boldsymbol{n} \cdot(\boldsymbol{\sigma} \boldsymbol{\delta} \boldsymbol{u}) d \Gamma=\int_{\Omega} \nabla \cdot(\boldsymbol{\sigma} \boldsymbol{\delta} \boldsymbol{u}) d \Omega
\end{gathered}
$$




$$
\int_{\Omega} \boldsymbol{\sigma} \cdot(\nabla \boldsymbol{\delta} \boldsymbol{u}) d \Omega=\int_{\Omega} \boldsymbol{b} \cdot \boldsymbol{\delta} \boldsymbol{u} d \Omega+\int_{\Gamma} \boldsymbol{t} \cdot \boldsymbol{\delta} \boldsymbol{u} d \Gamma
$$

Equation 2.19 can be expressed in terms of the strain tensor by using Equation 2.20.

$$
\boldsymbol{\sigma} \cdot(\nabla \boldsymbol{\delta} \boldsymbol{u})=\boldsymbol{\sigma}: \boldsymbol{\epsilon}(\boldsymbol{\delta u})
$$

By substituting Equation 2.20 in Equation 2.19, Equation 2.21 is obtained.

$$
\int_{\Omega} \boldsymbol{\sigma}: \boldsymbol{\epsilon}(\boldsymbol{\delta} \boldsymbol{u}) d \Omega=\int_{\Omega} \boldsymbol{b} \cdot \boldsymbol{\delta} \boldsymbol{u} d \Omega+\int_{\Gamma} \boldsymbol{t} \cdot \boldsymbol{\delta} \boldsymbol{u} d \Gamma
$$

\subsection{Isotropic Linearly Elastic Solid}

For a linearly elastic solid (or Hookean elastic solid), the relation between loading and deformation is linear, the rate of load application is not important, the deformations disappear completely upon removal of the loading, and the deformations are very small. Elastomers under small deformation can be modelled as linearly elastic solids.

In addition, if material properties can be described without reference to directions, the material is said to be isotropic. The constitutive equation for an isotropic linearly elastic solid can be described in term of two material properties as the first and second Lamé parameters $\lambda$ and $\mu$, respectively, according to Equation 2.22.

$$
\boldsymbol{\sigma}(\boldsymbol{u})=\lambda \operatorname{tr}(\boldsymbol{\epsilon}(\boldsymbol{u})) \boldsymbol{I}+2 \mu \boldsymbol{\epsilon}(\boldsymbol{u})
$$

Other material properties can also be chosen, such as the Young's modulus $E_{Y}$ and Poisson's ratio $\nu$, and the shear modulus $G$ and the bulk modulus $K$. The relation between these parameters and the Lamé parameters is presented in Equations 2.23, 2.24, and 2.25. The derivation of Equation 2.25 is presented in Appendix A.

$$
\begin{gathered}
\lambda=\frac{\nu E_{Y}}{(1+\nu)(1-2 \nu)} \\
G=\mu=\frac{E_{Y}}{2(1+\nu)}
\end{gathered}
$$




$$
K= \begin{cases}\frac{E_{Y}}{3(1-2 \nu)} & \text { for 3D problems } \\ \frac{E_{Y}}{2(1+\nu)(1-2 \nu)} & \text { for 2D plane strain } \\ \frac{E_{Y}}{2(1-2 \nu)} & \text { for 2D plane stress }\end{cases}
$$

\subsection{Incompressibility}

Soft robots, actuators, and sensors are commonly fabricated from incompressible elastomeric materials. For an incompressible material, the volume does not change under any state of stress. This consideration can be used in the equilibrium equations to model incompressibility.

For an isotropic linearly elastic solid, it is possible to invert Equation 2.22 to write the strain components in terms of the stress components according to Equation 2.26.

$$
\boldsymbol{\epsilon}(\boldsymbol{u})=\frac{1}{2 \nu}\left[\boldsymbol{\sigma}(\boldsymbol{u})-\frac{\lambda}{3 \lambda+2 \mu} \operatorname{tr}(\boldsymbol{\sigma}(\boldsymbol{u})) \boldsymbol{I}\right]
$$

The strain components can also be written in terms of Young's modulus $E_{Y}$ and Poisson's ratio $\nu$ according to Equation 2.27.

$$
\boldsymbol{\epsilon}(\boldsymbol{u})=\frac{1}{E_{Y}}[(1+\nu) \boldsymbol{\sigma}(\boldsymbol{u})-\nu \operatorname{tr}(\boldsymbol{\sigma}(\boldsymbol{u})) \boldsymbol{I}]
$$

By using Equation 2.27, the dilatation for an isotropic linearly elastic solid is then given by Equation 2.28.

$$
e(\boldsymbol{u})=\frac{1}{E_{Y}}(1-2 \nu) \operatorname{tr}(\boldsymbol{\sigma}(\boldsymbol{u}))
$$

For an incompressible material, the volume change (dilatation) is zero. As the volume does not change under any stress state, the stress components are not necessarily zero. So, the term $(1-2 \nu)$ must be zero and the Poisson's ratio is given by Equation 2.29.

$$
\nu=\frac{1}{2}
$$

By applying Equation 2.29 to Equation 2.25, it is possible to obtain the bulk modulus for incompressible materials according to Equation 2.30.

$$
K=\infty
$$

Equation 2.29 can be used to simulate nearly incompressible materials by using $\nu=0.49$. 


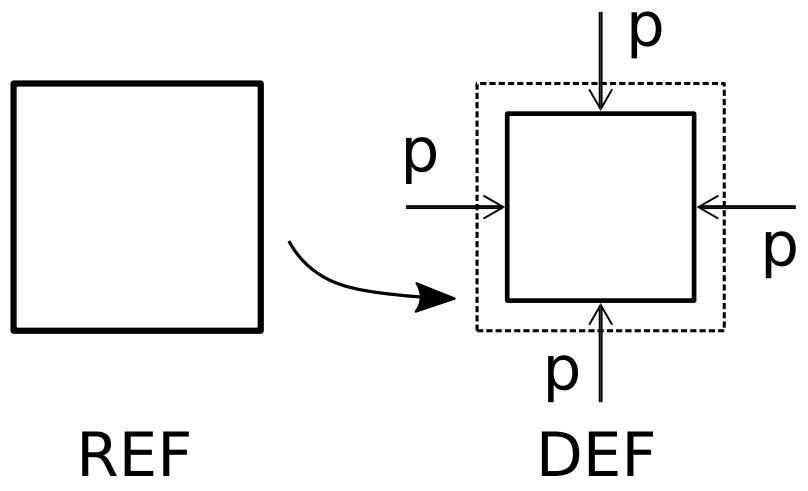

Figure 2.2 - Illustration of the relation between pressure $p$ and dilatation $\boldsymbol{\nabla} \cdot \boldsymbol{u}$ in 2D. The REF represents the reference configuration and the DEF represents the deformed configuration (Equation 2.31).

In this way, it is possible to evaluate aspects of incompressibility with isotropic linearly elastic models.

\subsection{Mixed u-p formulation}

By considering the deformation of a continuum subjected to a hydrostatic pressure load (Figure 2.2), it is possible to relate the pressure $p$ to the dilatation $\boldsymbol{\nabla} \cdot \boldsymbol{u}$ according to Equation 2.31 by using the bulk modulus $K$. With Equation 2.31 , it is possible to state the static equilibrium problem of Equation 2.21 in a mixed displacement-pressure ( $u-p)$ form.

$$
p=-K \nabla \cdot \boldsymbol{u}=-K \operatorname{tr}(\boldsymbol{\epsilon}(\boldsymbol{u}))
$$

By multiplying Equation 2.31 by a scalar test function $\delta p$ and by integrating it over the domain $\Omega$, the integral form is obtained according to Equation 2.32.

$$
\int_{\Omega} \delta p(p / K+\operatorname{tr}(\boldsymbol{\epsilon}(\boldsymbol{u}))) d \Omega=0
$$

The stress tensor in terms of shear modulus $G$ and bulk modulus $K$ is given by Equation 2.33 where $\epsilon^{\prime}$ is the deviatoric strain tensor defined in Equation 2.34. The deviatoric strain is the part of the strain that is not the hydrostatic strain.

$$
\begin{gathered}
\boldsymbol{\sigma}(\boldsymbol{u}, p)=2 G \boldsymbol{\epsilon}^{\prime}(\boldsymbol{u})-\boldsymbol{I} p \\
\boldsymbol{\epsilon}^{\prime}(\boldsymbol{u})= \begin{cases}\boldsymbol{\epsilon}(\boldsymbol{u})-\frac{1}{2} \boldsymbol{I} \nabla \cdot \boldsymbol{u} & \text { for 2D problems } \\
\boldsymbol{\epsilon}(\boldsymbol{u})-\frac{1}{3} \boldsymbol{I} \nabla \cdot \boldsymbol{u} & \text { for 3D problems }\end{cases}
\end{gathered}
$$


The static equilibrium in mixed form is defined by the system of Equation 2.35.

$$
\begin{aligned}
& \int_{\Omega} 2 G \boldsymbol{\epsilon}^{\prime}(\boldsymbol{u}): \boldsymbol{\epsilon}(\boldsymbol{\delta} \boldsymbol{u}) d \Omega-\int_{\Omega} p \boldsymbol{I}: \boldsymbol{\epsilon}(\boldsymbol{\delta} \boldsymbol{u}) d \Omega=\int_{\Omega} \boldsymbol{b} \cdot \boldsymbol{\delta} \boldsymbol{u} d \Omega+\int_{\Gamma} \boldsymbol{t} \cdot \boldsymbol{\delta} \boldsymbol{u} d \Gamma \\
& \int_{\Omega} \delta p(p / K+\operatorname{tr}(\boldsymbol{\epsilon}(\boldsymbol{u}))) d \Omega=0
\end{aligned}
$$

\subsection{Finite Element Method}

\subsubsection{Mixed displacement-pressure equations}

The finite element equations are obtained by discretizing the displacement $\boldsymbol{u}$ and pressure $p$ fields at each element according to Equation 2.36 where $\hat{\boldsymbol{u}}_{\boldsymbol{e}}$ and $\hat{p}_{\boldsymbol{e}}$ are the approximations of the elements fields $\boldsymbol{u}_{\boldsymbol{e}}$ and $p_{e}$, and $\tilde{\boldsymbol{u}}_{\boldsymbol{e}}$ and $\tilde{\boldsymbol{p}}_{\boldsymbol{e}}$ are the vectors of degrees of freedom of the approximation. The vector $\boldsymbol{N}_{\boldsymbol{p}}$ and matrix $\boldsymbol{N}_{\boldsymbol{u}}$ contain the pressure and displacement shape functions respectively.

$$
\begin{array}{r}
\boldsymbol{u}_{\boldsymbol{e}}(\boldsymbol{x}) \approx \hat{\boldsymbol{u}}_{\boldsymbol{e}}(\boldsymbol{x})=\boldsymbol{N}_{\boldsymbol{u}} \tilde{\boldsymbol{u}}_{\boldsymbol{e}} \\
p_{e}(\boldsymbol{x}) \approx \hat{p}_{e}(\boldsymbol{x})=\boldsymbol{N}_{\boldsymbol{p}} \tilde{\boldsymbol{p}}_{\boldsymbol{e}}
\end{array}
$$

It is possible to rewrite Equation 2.35 by using Voigt notation (ZIENKIEWICZ; TAYLOR, 2000) because the stress tensor is symmetric and the strain tensor is symmetric for small deformations. This approach reduces the order of the stress and strain tensor and it enables the representation of the double-dot product as a vector product according to Equation 2.37 where $\sigma_{V}$ is the vector representing the stress tensor and $\epsilon_{V}$ is the vector representing the strain tensor.

$$
\begin{aligned}
& \boldsymbol{\sigma}: \boldsymbol{\epsilon}=\boldsymbol{\sigma}_{\boldsymbol{V}}^{\boldsymbol{T}} \boldsymbol{\epsilon}_{\boldsymbol{V}}=\sigma_{11} \epsilon_{11}+\sigma_{22} \epsilon_{22}+\sigma_{33} \epsilon_{33}+2 \sigma_{12} \epsilon_{12}+2 \sigma_{13} \epsilon_{13}+2 \sigma_{23} \epsilon_{23} \\
& \boldsymbol{\sigma}_{\boldsymbol{V}}=\left\{\begin{array}{rr}
\left\{\sigma_{11}, \sigma_{22}, \sigma_{12}\right\}^{T}, & \text { for 2D problems } \\
\left\{\sigma_{11}, \sigma_{22}, \sigma_{33}, \sigma_{23}, \sigma_{13}, \sigma_{12}\right\}^{T}, & \text { for 3D problems }
\end{array}\right. \\
& \boldsymbol{\epsilon}_{\boldsymbol{V}}=\left\{\begin{array}{rr}
\left\{\epsilon_{11}, \epsilon_{22}, 2 \epsilon_{12}\right\}^{T}, & \text { for 2D problems } \\
\left\{\epsilon_{11}, \epsilon_{22}, \epsilon_{33}, 2 \epsilon_{23}, 2 \epsilon_{13}, 2 \epsilon_{12}\right\}^{T}, & \text { for 3D problems }
\end{array}\right.
\end{aligned}
$$

The discretization of Equations 2.36 are used to compute the discretized versions of other fields like the strain field of Equation 2.38 and the volumetric deformation field 2.39. The 
matrix $\boldsymbol{B}$ is obtained by differentiating and combining rows of matrix $\boldsymbol{N}_{\boldsymbol{u}}$ (BATHE, 1995).

$$
\epsilon_{V}=B \tilde{u}_{e}
$$

The volumetric deformation is approximated by using the discretization of the strain tensor according to Equation 2.39 where $\boldsymbol{m}$ is a vector equivalent to the identity matrix for Voigt notation and it is defined in Equation 2.40.

$$
\begin{gathered}
\nabla \cdot \boldsymbol{u}=\operatorname{tr}(\boldsymbol{\epsilon}(\boldsymbol{u}))=\boldsymbol{m}^{\boldsymbol{T}} \boldsymbol{\epsilon}_{\boldsymbol{V}}=\boldsymbol{m}^{\boldsymbol{T}} \boldsymbol{B} \tilde{\boldsymbol{u}}_{\boldsymbol{e}} \\
\boldsymbol{m}=\left\{\begin{array}{rr}
\{1,1,0\}^{T}, & \text { for 2D problems } \\
\{1,1,1,0,0,0\}^{T}, & \text { for 3D problems }
\end{array}\right.
\end{gathered}
$$

The test functions $\boldsymbol{\delta} \boldsymbol{u}$ and $\delta p$ of Equation 2.35 are also approximated at each element by using the same shape functions used for the approximation of trial functions $\boldsymbol{u}$ and $p$ according to Equation 2.41.

$$
\begin{array}{r}
\boldsymbol{\delta} \boldsymbol{u}_{e}(\boldsymbol{x}) \approx \boldsymbol{N}_{\boldsymbol{u}} \boldsymbol{\delta} \tilde{\boldsymbol{u}} \\
\delta p_{e}(\boldsymbol{x}) \approx \boldsymbol{N}_{\boldsymbol{p}} \boldsymbol{\delta} \tilde{\boldsymbol{p}}
\end{array}
$$

The deviatoric strain tensor is also symmetric, so it can represented as vector by using Voigt notation. As Equation 2.35 presents the double-dot product of the strain tensor by the deviatoric strain tensor, the diagonal term of the deviatoric strain tensor will not be multiplied by 2 according to Equation 2.42 that illustrates the two dimensional (2D) case.

$$
\begin{array}{r}
\boldsymbol{e}_{\boldsymbol{V}}^{\prime}=\left[\begin{array}{c}
\epsilon_{11}^{\prime} \\
\epsilon_{22}^{\prime} \\
\epsilon_{12}^{\prime}
\end{array}\right]=\left[\begin{array}{lll}
1 & 0 & 0 \\
0 & 1 & 0 \\
0 & 0 & \frac{1}{2}
\end{array}\right] \boldsymbol{e}_{\boldsymbol{V}}-\frac{1}{2} \boldsymbol{m} \boldsymbol{m}^{\boldsymbol{T}} \boldsymbol{e}_{\boldsymbol{V}}=\left(\boldsymbol{I}_{\mathbf{0}}-\frac{1}{2} \boldsymbol{m} \boldsymbol{m}^{\boldsymbol{T}}\right) \boldsymbol{B} \tilde{\boldsymbol{u}}_{\boldsymbol{e}} \\
2 G \boldsymbol{e}_{\boldsymbol{V}}^{\prime}=2 G\left(\boldsymbol{I}_{\mathbf{0}}-\frac{1}{2} \boldsymbol{m} \boldsymbol{m}^{\boldsymbol{T}}\right) \boldsymbol{B} \tilde{\boldsymbol{u}}_{\boldsymbol{e}}=\boldsymbol{D}_{\boldsymbol{d}} \boldsymbol{B} \tilde{\boldsymbol{u}}_{\boldsymbol{e}}
\end{array}
$$

The same procedure is used for the three dimensional (3D) case and Equation 2.43 is obtained for the matrix $\boldsymbol{D}_{\boldsymbol{d}}$.

$$
\boldsymbol{D}_{\boldsymbol{d}}=\left\{\begin{array}{cc}
2 G\left(\boldsymbol{I}_{\mathbf{0}}-\frac{1}{2} \boldsymbol{m} \boldsymbol{m}^{\boldsymbol{T}}\right), & \text { for 2D problems } \\
2 G\left(\boldsymbol{I}_{\mathbf{0}}-\frac{1}{3} \boldsymbol{m} \boldsymbol{m}^{\boldsymbol{T}}\right), & \text { for 3D problems }
\end{array}\right.
$$


By writing the first equation of problem 2.35 in Voigt notation and by substituting the discretized relations, Equation 2.44 is obtained.

$$
\begin{aligned}
& \int_{\Omega} \boldsymbol{\delta} \boldsymbol{\epsilon}_{\boldsymbol{V}}^{\boldsymbol{T}} 2 G \boldsymbol{\epsilon}_{V}^{\prime} d \Omega-\int_{\Omega} \boldsymbol{\delta} \boldsymbol{\epsilon}_{V}^{\boldsymbol{T}} \boldsymbol{m} p d \Omega=\int_{\Omega} \boldsymbol{\delta} \boldsymbol{u}^{\boldsymbol{T}} \boldsymbol{b} d \Omega+\int_{\Gamma} \boldsymbol{\delta} \boldsymbol{u}^{\boldsymbol{T}} \boldsymbol{t} d \Gamma \\
& \int_{\Omega_{e}} \boldsymbol{\delta} \tilde{\boldsymbol{u}}_{\boldsymbol{e}}^{\boldsymbol{T}} \boldsymbol{B}^{\boldsymbol{T}} \boldsymbol{D}_{\boldsymbol{d}} \boldsymbol{B} \tilde{\boldsymbol{u}}_{\boldsymbol{e}} d \Omega-\int_{\Omega_{e}} \boldsymbol{\delta} \tilde{\boldsymbol{u}}_{\boldsymbol{e}}^{\boldsymbol{T}} \boldsymbol{B}^{\boldsymbol{T}} \boldsymbol{m} \boldsymbol{N}_{p} \tilde{\boldsymbol{p}}_{e} d \Omega=\int_{\Omega_{e}} \boldsymbol{\delta} \tilde{\boldsymbol{u}}_{\boldsymbol{e}}^{\boldsymbol{T}} \boldsymbol{N}_{\boldsymbol{u}}^{\boldsymbol{T}} \boldsymbol{b} d \Omega+\int_{\Gamma_{e}} \boldsymbol{\delta} \tilde{\boldsymbol{u}}_{\boldsymbol{e}}^{\boldsymbol{T}} \boldsymbol{N}_{\boldsymbol{u}}^{\boldsymbol{T}} \boldsymbol{t} d \Gamma \\
& \boldsymbol{\delta} \tilde{\boldsymbol{u}}_{\boldsymbol{e}}^{\boldsymbol{T}}\left(\int_{\Omega_{e}} \boldsymbol{B}^{\boldsymbol{T}} \boldsymbol{D}_{\boldsymbol{d}} \boldsymbol{B} \tilde{\boldsymbol{u}}_{\boldsymbol{e}} d \Omega-\int_{\Omega_{e}} \boldsymbol{B}^{\boldsymbol{T}} \boldsymbol{m} \boldsymbol{N}_{\boldsymbol{p}} \tilde{\boldsymbol{p}}_{\boldsymbol{e}} d \Omega\right)=\boldsymbol{\delta} \tilde{\boldsymbol{u}}_{\boldsymbol{e}}^{\boldsymbol{T}}\left(\int_{\Omega_{e}} \boldsymbol{N}_{u}^{\boldsymbol{T}} \boldsymbol{b} d \Omega+\int_{\Gamma_{e}} \boldsymbol{N}_{u}^{\boldsymbol{T}} \boldsymbol{t} d \Gamma\right)
\end{aligned}
$$

As Equation 2.35 has to hold for any arbitrary test function $\delta \tilde{\boldsymbol{u}}_{\boldsymbol{e}}$ and the vectors of degrees of freedom $\tilde{\boldsymbol{u}}_{\boldsymbol{e}}$ and $\tilde{\boldsymbol{p}}_{\boldsymbol{e}}$ are independent from the coordinates $\boldsymbol{x}$, Equation 2.45 is obtained.

$$
\left(\int_{\Omega_{e}} \boldsymbol{B}^{T} \boldsymbol{D}_{\boldsymbol{d}} \boldsymbol{B} d \Omega\right) \tilde{\boldsymbol{u}}_{\boldsymbol{e}}+\left(\int_{\Omega_{e}} \boldsymbol{B}^{T} \boldsymbol{m} \boldsymbol{N}_{\boldsymbol{p}} d \Omega\right) \tilde{\boldsymbol{p}}_{\boldsymbol{e}}=\left(\int_{\Omega_{e}} \boldsymbol{N}_{u}^{\boldsymbol{T}} \boldsymbol{b} d \Omega+\int_{\Gamma_{e}} \boldsymbol{N}_{u}^{\boldsymbol{T}} \boldsymbol{t} d \Gamma\right)
$$

By repeating the same procedure for the second equation of the problem 2.35, Equation 2.46 is found.

$$
\begin{array}{r}
\int_{\Omega_{e}} \boldsymbol{N}_{\boldsymbol{p}} \boldsymbol{\delta} \tilde{\boldsymbol{p}}_{\boldsymbol{e}}\left(\boldsymbol{N}_{\boldsymbol{p}} \tilde{\boldsymbol{p}}_{\boldsymbol{e}} / K+\boldsymbol{m}^{\boldsymbol{T}} \boldsymbol{B} \tilde{\boldsymbol{u}}_{\boldsymbol{e}}\right) d \Omega=0 \\
\boldsymbol{\delta} \tilde{\boldsymbol{p}}_{\boldsymbol{e}}^{T}\left(\int_{\Omega_{e}} \boldsymbol{N}_{\boldsymbol{p}}^{T}\left(\boldsymbol{N}_{\boldsymbol{p}} \tilde{\boldsymbol{p}}_{\boldsymbol{e}} / K+\boldsymbol{m}^{\boldsymbol{T}} \boldsymbol{B} \tilde{\boldsymbol{u}}_{\boldsymbol{e}}\right) d \Omega\right)=0
\end{array}
$$

As Equation 2.46 has to hold for arbitrary test functions $\delta \tilde{\boldsymbol{p}}_{\boldsymbol{e}}$, the integral part of equation must be equal to zero. It is possible to rearrange the resulting equation to isolate the degrees of freedom vector according to Equation 2.47.

$$
\left(\int_{\Omega_{e}} \boldsymbol{N}_{\boldsymbol{p}}^{T} \boldsymbol{m}^{\boldsymbol{T}} \boldsymbol{B} d \Omega\right) \tilde{\boldsymbol{u}}_{\boldsymbol{e}}+\left(\int_{\Omega_{e}} \boldsymbol{N}_{\boldsymbol{p}}^{T} \frac{1}{K} \boldsymbol{N}_{\boldsymbol{p}} d \Omega\right) \tilde{\boldsymbol{p}}_{\boldsymbol{e}}=0
$$

From Equations 2.45 and 2.47, it is possible to define the stiffness matrix $\boldsymbol{K}_{\boldsymbol{F}}$ (Equation 2.48), the coupling matrix $\boldsymbol{C}_{\boldsymbol{F}}$ (Equation 2.49), the matrix $\boldsymbol{D}_{\boldsymbol{F}}$ (Equation 2.50), and the load vector $\boldsymbol{f}_{\boldsymbol{F}}$ (Equation 2.51). These tensors are assembled by summing the contribution of all finite elements.

$$
\boldsymbol{K}_{\boldsymbol{F}}=\sum_{e}\left(\int_{\Omega_{e}} \boldsymbol{B}^{\boldsymbol{T}} \boldsymbol{D}_{\boldsymbol{d}} \boldsymbol{B} d \Omega\right)
$$




$$
\begin{gathered}
\boldsymbol{C}_{\boldsymbol{F}}=\sum_{e}\left(\int_{\Omega_{e}} \boldsymbol{B}^{\boldsymbol{T}} \boldsymbol{m} \boldsymbol{N}_{\boldsymbol{p}} d \Omega\right) \\
\boldsymbol{D}_{\boldsymbol{F}}=\sum_{e}\left(\int_{\Omega_{e}} \boldsymbol{N}_{\boldsymbol{p}}^{\boldsymbol{T}} \frac{1}{K} \boldsymbol{N}_{\boldsymbol{p}} d \Omega\right) \\
\boldsymbol{f}_{\boldsymbol{F}}=\sum_{e}\left(\int_{\Omega_{e}} \boldsymbol{N}_{\boldsymbol{u}}^{\boldsymbol{T}} \boldsymbol{b} d \Omega+\int_{\Gamma_{e}} \boldsymbol{N}_{\boldsymbol{u}}^{\boldsymbol{T}} \boldsymbol{t} d \Gamma\right)
\end{gathered}
$$

So, the linear system of Equation 2.52 is obtained. It can be used to find the global vectors of degrees of freedom $\tilde{\boldsymbol{u}}$ and $\tilde{\boldsymbol{p}}$ which are the solution to this finite element problem.

$$
\left[\begin{array}{cc}
K_{F} & C_{F} \\
C_{F}^{T} & D_{F}
\end{array}\right]\left\{\begin{array}{l}
\tilde{u} \\
\tilde{p}
\end{array}\right\}=\left\{\begin{array}{c}
f_{F} \\
0
\end{array}\right\}
$$

\subsection{Topology Optimization Method}

As discussed in Introduction (Section 1.2), the optimization approach used in this work is topology optimization due to its generality.

Topology optimization was introduced by Bendsøe and Kikuchi (1988) to solve minimum compliance problems. The objective of the authors is to develop a technique capable of designing structures that are not topologically equivalent to the initial guess, i.e., to create a technique able to insert and remove holes of the design. Additionally, the developed method avoids remeshing techniques and it is easy to extend to three dimensional (3D) problems.

In Bendsøe and Kikuchi (1988), topology optimization is solved by using homogenization techniques, i.e., by describing the macroscopic behaviour of the material in terms of its microscopic properties. Since then, several approaches to topology optimization have been proposed such as density-based in Bendsøe (1989), level set in Allaire, Jouve and Toader (2002), phase field in Bourdin and Chambolle (2003), topological derivative in Sokolowski and Zochowski (1999), and evolutionary approaches in Xie and Steven (1993). This dissertation is concerned with the density-based technique which consists of assigning a density value $\rho$ to each pixel (2D problems) or voxel (3D problems) of the discrete domain. The density value $\rho$ is used to interpolate material properties. For the classical minimum compliance problem, for example, $\rho=1$ corresponds to solid domain and $\rho=0$ to void domain. For other problems, such as the design-dependent load problem solved in this work, the density values may be related to other domains such as $\rho=1$ for solid domain and $\rho=0$ for fluid domain. Equation 
2.53 defines the density variable $\rho$.

$$
\rho= \begin{cases}1, & \text { for solid } \\ 0, & \text { for void or fluid }\end{cases}
$$

However, Equation 2.53 defines a discrete design variable and discrete optimization problems are difficult to solve for large scale problems, such as topology optimization (which may present thousands and up to millions design variables), because integer optimization methods are not well-suited for large scale problems (SIGMUND; MAUTE, 2013). Also, discrete optimization problems present multiple local minima and mesh dependent solutions.

An alternative to enable the use of continuous optimization algorithms is to relax the problem by allowing the design variable $\rho$ to be a continuous variable $\rho \in[0,1]$ and to use penalization techniques to avoid intermediate densities as proposed by (BENDSØE, 1989), because intermediate densities may be undesired due to manufacturing constraints. Penalization techniques can be used with relaxation methods to promote the convergence to almost discrete designs. The SIMP method (Solid Isotropic Material with Penalization) proposed by Bendsøe (1989) is an example of penalization approach. For minimum compliance problems, intermediate densities contribute less to the object function (stiffness) than to the volume of material because the power of a value $\rho \in] 0,1[$ is lower than $\rho$ itself.

In SIMP, the Young's modulus field is considered dependent on density $\rho$ according to Equation 2.54 where $E_{S}$ is the Young's modulus of solid material, $E_{V}$ is a small value introduced to avoid $E_{Y}(\rho)=0$ in void regions, and $z$ is a penalization factor. The Poisson's ratio is considered independent of $\rho$.

$$
E_{Y}(\rho)=E_{V}+\rho^{z}\left(E_{S}-E_{V}\right)
$$

The penalization parameter of SIMP has to be properly selected. Low values of $z$ causes too much grey scale while high values of $z$ causes fast convergence to local minima. According to Sigmund and Maute (2013), these properties motivates the use of continuation techniques and they result in convergence to better designs even for non compliance minimization problems. However, convergence cannot be guaranteed.

The topology optimization problem consists of minimizing (or maximizing) a functional $J(\boldsymbol{u}, \boldsymbol{\rho})$ by the automatic selection of densities (design variables) that satisfies the equilibrium 
equations $F(\boldsymbol{u}, \boldsymbol{\rho})$ and volume constraint $V^{*}$ according to Equation 2.55.

$$
\begin{array}{cl}
\max _{\boldsymbol{\rho}} & J(\boldsymbol{u}, \boldsymbol{\rho}) \\
\text { s.t. } & \boldsymbol{F}(\boldsymbol{u}, \boldsymbol{\rho})=\mathbf{0} \\
& g(\rho)=V^{*}-\int_{\Omega} \rho d \Omega \geq 0 \\
& \mathbf{0} \leq \boldsymbol{\rho} \leq \mathbf{1}
\end{array}
$$

To ensure well-posed and mesh independent solutions, it is necessary to introduce constraints to Equation 2.55 in the form of perimeter control, gradient control, sensitivity filters, density filters and projections, for example. The density filter used in this work is defined by Equation 2.56 in which $\bar{\rho}$ is the filtered variable and $r$ is the length parameter. This density filter is proposed by Lazarov and Sigmund (2010).

$$
\begin{aligned}
-r^{2} \nabla^{2} \bar{\rho}+\bar{\rho}=\rho, & \text { in } \Omega \\
\nabla \bar{\rho} \cdot \boldsymbol{n} & =0, \quad \text { on } \partial \Omega
\end{aligned}
$$

Equation 2.56 can be written in weak form by multiplying it by a test function $\delta \bar{\rho}$, integrating over the domain $\Omega$ and applying Green's theorem. The result is Equation 2.57.

$$
r^{2} \int_{\Omega} \nabla \bar{\rho} \cdot \nabla(\delta \bar{\rho}) d \Omega+\int_{\Omega} \bar{\rho}(\delta \bar{\rho}) d \Omega=\int_{\Omega} \rho(\delta \bar{\rho}) d \Omega
$$

The design variable and filtered fields of Equation 2.57 must be discretized by first or higher order elements because Equation 2.57 presents gradients of $\rho$ and $\bar{\rho}$. In this work, they are discretized by first order elements as discussed in Chapter 3.

The density filter introduces gray transition regions which can be reduced by the application of a threshold projection. The form of projection used in this work is presented in Equation 2.58 according to what is proposed by Wang, Lazarov and Sigmund (2010). This equation is a shorter and simpler form of the projection suggested by Xu, Cai and Cheng (2009) based on the works of Guest, Prévost and Belytschko (2004) and Sigmund (2007). The parameter $\eta$ is the threshold of the projection, i.e., density values above $\eta$ are projected to 1 and density values below $\eta$ are projected to 0 . The parameter $\beta$ is the slope of the projection curve, so for lower values of $\beta$ the curve is smooth while for higher values of $\beta$ the curve is steep. The graphical characteristics of the threshold projection are summarized in Figure 2.3.

$$
\hat{\rho}=\frac{\tanh (\beta \eta)+\tanh (\beta(\bar{\rho}-\eta))}{\tanh (\beta \eta)+\tanh (\beta(1-\eta))}
$$

When $\eta=0$, the combination of density filter and threshold projection ensures minimum 


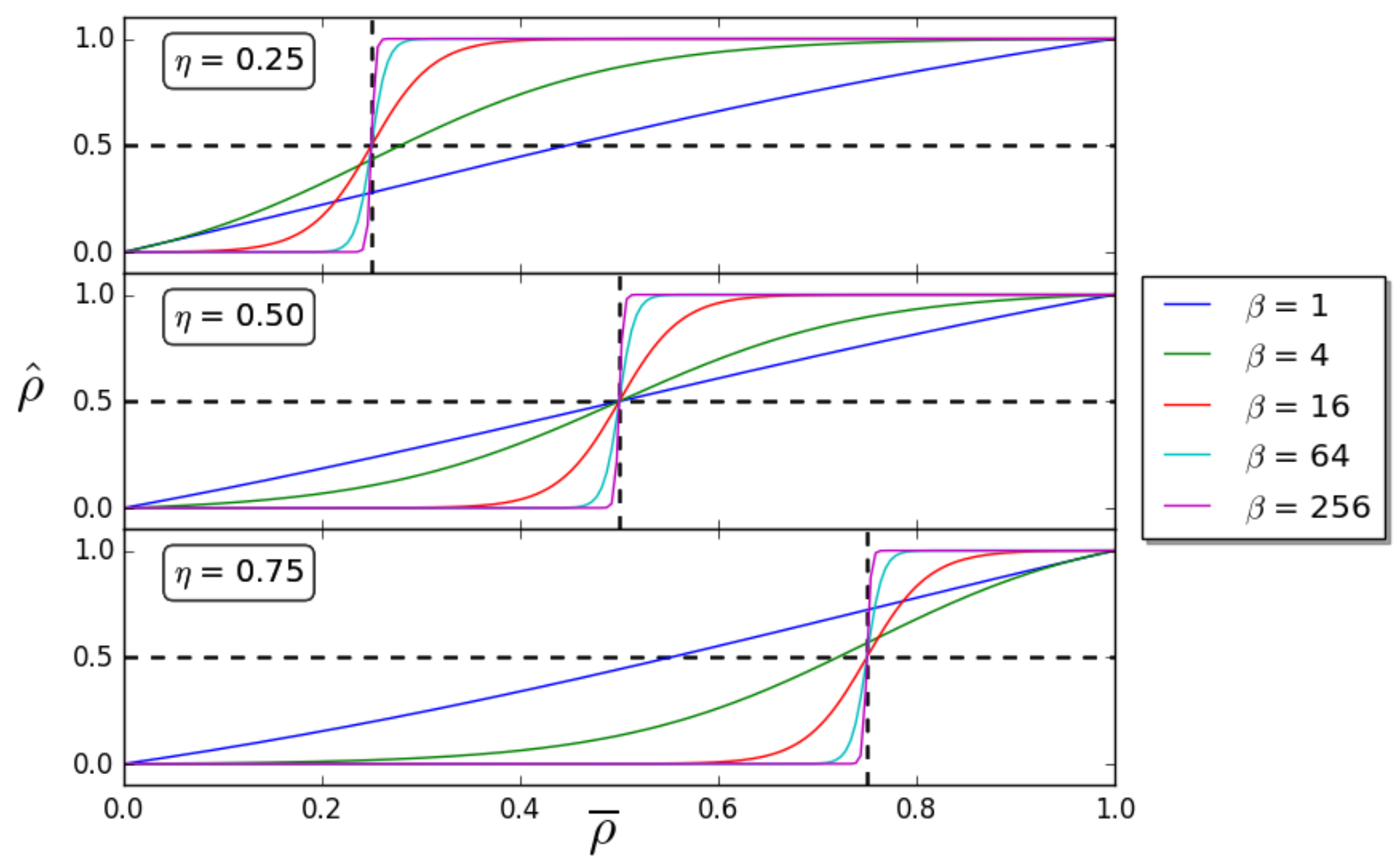

Figure 2.3 - Threshold projection (Equation 2.58) for different values of $\eta$ and $\beta$. The parameter $\eta$ controls the threshold of projection and the parameter $\beta$ controls the slope of the curve.

length scale at solid phase. When $\eta=1$, the combination ensures minimum length scale at void phase. In this work, the value of $\eta=0.5$ is used for smoother convergence according to Wang, Lazarov and Sigmund (2010).

After filtering and projecting the design variables, the physical density field becomes $\hat{\rho}$. Therefore, material properties interpolations, such as SIMP, must use $\hat{\rho}$ instead of $\rho$ according to Equation 2.59.

$$
E_{Y}(\hat{\rho})=E_{V}+\hat{\rho}^{z}\left(E_{S}-E_{V}\right)
$$

In this work, the discreteness of solutions is measured by Equation 2.60. This measure is introduced by Sigmund (2007) to evaluate quantitatively the amount of intermediate densities. In this equation, the maximum value for each element $e$ occurs for $\hat{\rho}_{e}=0.5$. Therefore, $M_{n d}$ is larger for intermediate densities. It is important to obtain lower values for $M_{n d}$ because there is no practical interest in intermediate densities.

$$
M_{n d}=\frac{\sum_{e=1}^{n} 4 \hat{\rho}_{e}\left(1-\hat{\rho}_{e}\right)}{n} \times 100 \%
$$

More information about numerical instabilities in topology optimization and the requirement 
of using relaxation and constraints methods is provided by Sigmund and Petersson (1998).

\subsubsection{Continuation}

A continuation technique is applied to avoid fast convergence to local minima with the objective of obtaining better optimization results. The continuation is applied to threshold projection parameter $\beta$ and to SIMP penalization parameter $z$. The continuation of $\beta$ starts with $\beta_{1}=1$ and the update law is to double the parameter at each continuation step according to Equation 2.61. The parameter stops to be updated at the maximum value of $\beta_{\max }$.

$$
\beta_{i+1}=\min \left(2 \beta_{i}, \beta_{\max }\right)
$$

The continuation for the SIMP parameter starts with $z_{1}=1$ and the update law is to increase the value of $z$ by one at each continuation step according to Equation 2.62. The maximum value of $z$ is $z_{\max }$.

$$
z_{i+1}=\min \left(z_{i}+1, z_{\max }\right)
$$

\subsubsection{Barrier Projection}

The barrier projection described in this section is the contribution of this dissertation and it is published in Souza and Silva (2020).

For the barrier projection approach, the design variable field is divided in three regions: exterior fluid, interior fluid, and solid. The idea is to separate exterior fluid from interior fluid with solid elements between then to avoid leaking designs. Each region is associated with a design variable value according to Equation 2.63.

$$
\rho=\left\{\begin{aligned}
1, & \text { for external fluid } \\
0, & \text { for solid } \\
-1, & \text { for internal fluid }
\end{aligned}\right.
$$

The design variable field is density filtered by an Helmholtz equation in order to avoid steep transitions between exterior and interior fluid domains according to Equation 2.64. The length parameter $l$ determines smoothing intensity.

$$
l^{2} \int_{\Omega} \nabla \bar{\rho} \cdot \nabla(\delta \bar{\rho}) d \Omega+\int_{\Omega} \bar{\rho}(\delta \bar{\rho}) d \Omega=\int_{\Omega} \rho(\delta \bar{\rho}) d \Omega
$$

The design variable values $-1,0$, and 1 are conveniently chosen so that the projection given by Equation 2.65 (for $\alpha$ even) can map interior and exterior fluid to the same value, what 


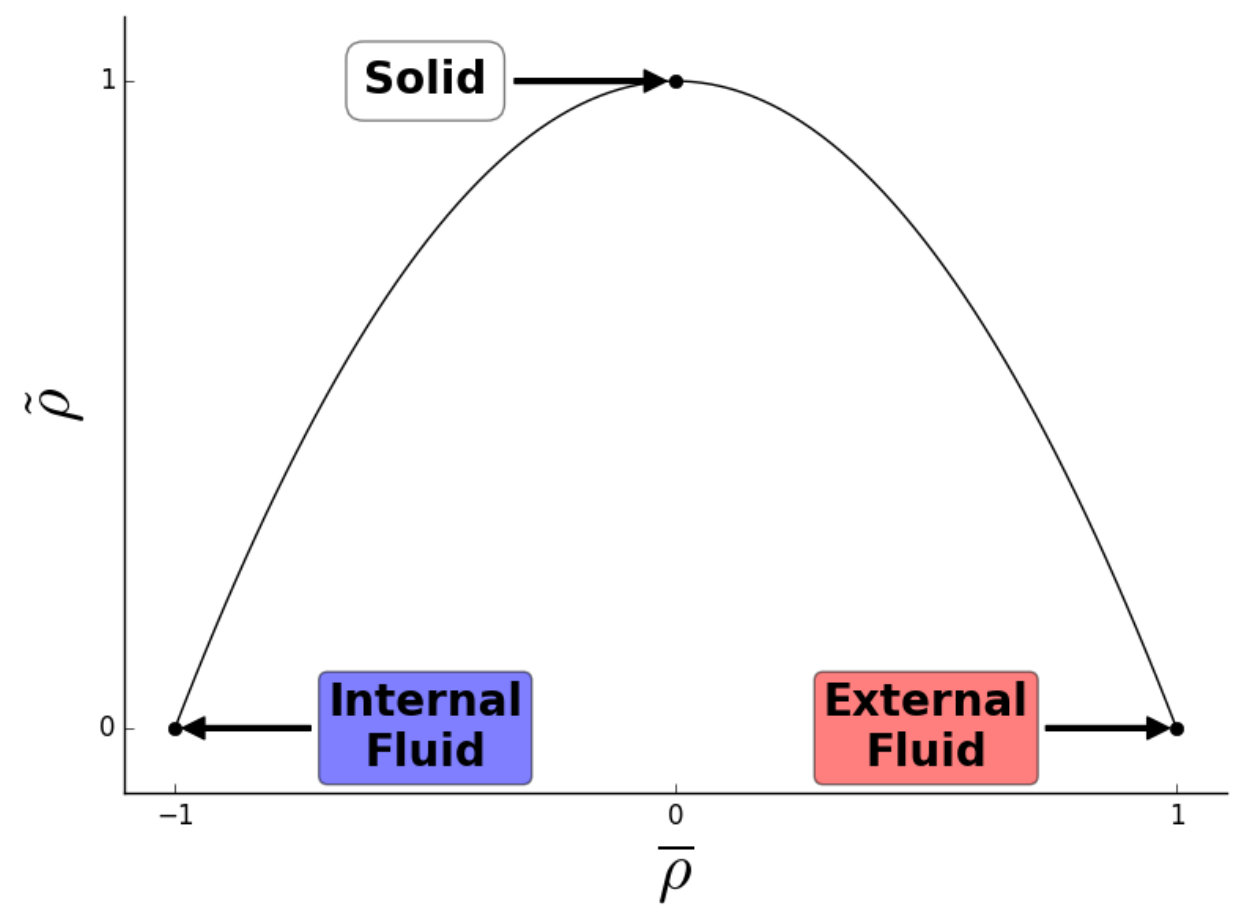

Figure $2.4-$ Barrier projection function for $\alpha=2$.

makes them have the same physical properties as illustrated by Figure 2.4. This work uses $\alpha=$ 2 , because it demonstrated better convergence than higher values.

$$
\tilde{\rho}=1-\bar{\rho}^{\alpha}
$$

Besides mapping exterior and interior fluid domains to the same values, the projection of Equation 2.65 introduces a concave region between exterior and interior fluid domains as illustrated by Figure 2.4. The concave region difficulties transitions between these domains. Also, the introduction of a exterior density inside a interior region would require the appearance of a solid contour around the exterior fluid density. The summation of these two effects makes only the actuator walls change its shape.

Then, the barrier variable goes through a density filter and a threshold projection steps to give the physical density field. Equations 2.66 and 2.67 are equivalent in form and function to Equations 2.57 and 2.58, however they are applied to different variables field. So, they are reproduced once again to highlight the difference between input and output fields.

$$
\begin{gathered}
r^{2} \int_{\Omega} \nabla \overline{\bar{\rho}} \cdot \nabla(\delta \overline{\bar{\rho}}) d \Omega+\int_{\Omega} \overline{\bar{\rho}}(\delta \overline{\bar{\rho}}) d \Omega=\int_{\Omega} \tilde{\rho}(\delta \overline{\bar{\rho}}) d \Omega \\
\hat{\rho}=\frac{\tanh (\beta \eta)+\tanh (\beta(\overline{\bar{\rho}}-\eta))}{\tanh (\beta \eta)+\tanh (\beta(1-\eta))}
\end{gathered}
$$




\subsubsection{Compliant Mechanisms}

Compliant mechanism are mechanical components that transmit mechanical energy (in the form of displacements or forces) from its input ports to its output ports by the deformation of its structure. They play similar roles than of its rigid body counterparts, but instead of moving by the relative displacement of joints, they move by the deformation of their body. As this is very similar to what soft robots and actuators do, it is possible to use compliant mechanism formulations to design soft robots and actuators.

According to Midha, Norton and Howell (1994), the monolithic structure of compliant mechanisms ensure less wear, noisy, and backlash making them adequate to precision and micrometer applications such as microelectromechanical systems (MEMS).

The compliant mechanism formulation applied in this dissertation is presented by Pedersen, Buhl and Sigmund (2001) in which the objective function to be maximized is the output displacement defined by Equation 2.68. The vector $\boldsymbol{l}$ gives the direction and orientation of the desired output displacement $(J(\boldsymbol{u}))$ and $\Gamma_{\text {out }}$ is the output port surface. The objective function has unit of area instead of displacement, because the displacement is integrated over the output port.

$$
J(\boldsymbol{u})=\int_{\Gamma_{\text {out }}} \boldsymbol{l} \cdot \boldsymbol{u} d \Gamma
$$

A spring of stiffness $k_{\text {out }}$ is placed at output port to model the elasticity of the work piece. The contribution of this spring to equilibrium equations can be found by the substitution of the spring by a surface load $\boldsymbol{F}_{e l}$ on the output port. The elastic force magnitude is equal to $k_{\text {out }}$ times the displacement component in the output direction. The orientation of the elastic force is opposed to the output displacement, so the elastic force vector is given by Equation 2.69.

$$
\boldsymbol{F}_{\text {el }}=-k_{\text {out }}(\boldsymbol{l} \cdot \boldsymbol{u}) \boldsymbol{l}
$$

The bilinear form associated to the contribution of the spring, $A_{e l}$, is found by multiplying the elastic force by a test function $\boldsymbol{\delta} \boldsymbol{u}$ and integrating over the output port $\Gamma_{\text {out }}$. The result is presented in Equation 2.70. The size of output port is given by the norm of the output port surface $\left\|\Gamma_{\text {out }}\right\|$. Equation 2.70 is added to right hand side of the first Equation of system 2.35 to include the contribution of the spring to equilibrium equations.

$$
A_{\text {el }}(\boldsymbol{u}, \boldsymbol{\delta} \boldsymbol{u})=-\frac{k_{\text {out }}}{\left\|\Gamma_{\text {out }}\right\|} \int_{\Gamma_{\text {out }}}(\boldsymbol{l} \cdot \boldsymbol{u})(\boldsymbol{l} \cdot \boldsymbol{\delta} \boldsymbol{u}) d \Gamma
$$

Several formulations have been proposed to design compliant mechanisms by topology optimization. For an in depth comparison between formulations see Deepak et al. (2009). 


\subsubsection{Design Dependent Loads}

Pressure is classified as a design dependent load because the forces applied to a continuum due to pressure load depend on the continuum geometry (i.e. depends on its design) as pressure always act normal to the surface. In topology optimization, design dependent loads pose an additional challenge because the design changes with the execution of the optimization routine. So, it is necessary to apply algorithms or models able to represent the load according to the design.

Chen and Kikuchi (2001) propose an algorithm that simulates design-dependent loads by using fictitious thermal loads. Hammer and Olhoff (2000), Du and Olhoff (2004a), Du and Olhoff (2004b) present a formulation to find iso-density surfaces that can be used to describe the load surface. Sigmund and Clausen (2007) propose the use of a mixed displacement pressure (u-p) formulation based on shear and bulk modulus. The equations from this formulation are used to transmit the pressure load from boundaries to the evolving surface. This idea is also studied by Bruggi and Cinquini (2009) using Johnson and Mercier finite elements.

The approach applied in this dissertation is the one proposed by Sigmund and Clausen (2007). According to them, different material behavior can be modelled by varying $G$ and $K$ according to Equation 2.71 .

$$
\begin{array}{rrrr}
K \text { finite, } & G \text { finite } & \rightarrow & \text { normal (compressible) elasticity } \\
K \gg G, & G \text { finite } & \rightarrow & \text { (almost) incompressible elasticity } \\
1 / K=0, & G \text { finite } & \rightarrow & \text { incompressible elasticity } \\
K \text { finite, } & G=0 & \rightarrow & \text { compressible inviscid hydrostatic fluid } \\
1 / K=0, & G=0 & \rightarrow & \text { incompressible inviscid hydrostatic fluid } \\
K \text { small, } & G=0 & \rightarrow & \text { void (air) }
\end{array}
$$

The material interpolation used is a modified SIMP method based on bulk and shear moduli in place of Young's modulus (SIGMUND; CLAUSEN, 2007), according to Equation 2.72 where $G_{s}$ and $K_{s}$ are the shear and bulk moduli of the solid and $G_{f}$ and $K_{f}$ are the shear and bulk moduli of the fluid.

$$
\begin{array}{r}
G(\hat{\rho})=G_{f}+\hat{\rho}^{z}\left(G_{s}-G_{f}\right) \\
K(\hat{\rho})=K_{f}+\hat{\rho}^{z}\left(K_{s}-K_{f}\right)
\end{array}
$$




\section{NUMERICAL IMPLEMENTATION}

This chapter presents the algorithm used to design actuators driven by pressure loads and its numerical implementation. It starts by describing the steps of the algorithm and how each step connects to each other. Then, the software packages used are described: FEniCS framework for FEM (ALN/ES et al., 2015), IPOPT package for nonlinear optimization (WäCHTER; BIEGLER, 2005), and dolfin-adjoint for sensitivity analysis (FARRELL et al., 2013; FUNKE; FARRELL, 2013). The development of sensitivity analysis is also presented in this chapter because the volume constraint must be explicit calculated when using dolfin-adjoint. This work uses the linear system solver MA57 from Duff (2004) and the software developed is written in Python language. The verification of the implementation in FEniCS is presented in Appendix D.

\subsection{Algorithm}

The topology optimization algorithm used to design actuators driven by pressure loads is implemented following the diagram of Figure 3.1. The projection step is a sequence of filters and projections that map design variables into physical densities. For traditional approach only the density filtering and threshold projection are used. For barrier projection approach all the steps are used.

The forward problem, smoothing, and density filter equations are solved with FEM by using the FEniCS framework (ALNÆES et al., 2015) which converts scientific models described by variational forms into efficient finite element code. FEniCS has interfaces in $\mathrm{C}++$ and Python for user level applications and, in this work, the Python interface is chosen to allow the use of dolfin-adjoint. More details about FEniCS implementation and its API (Application

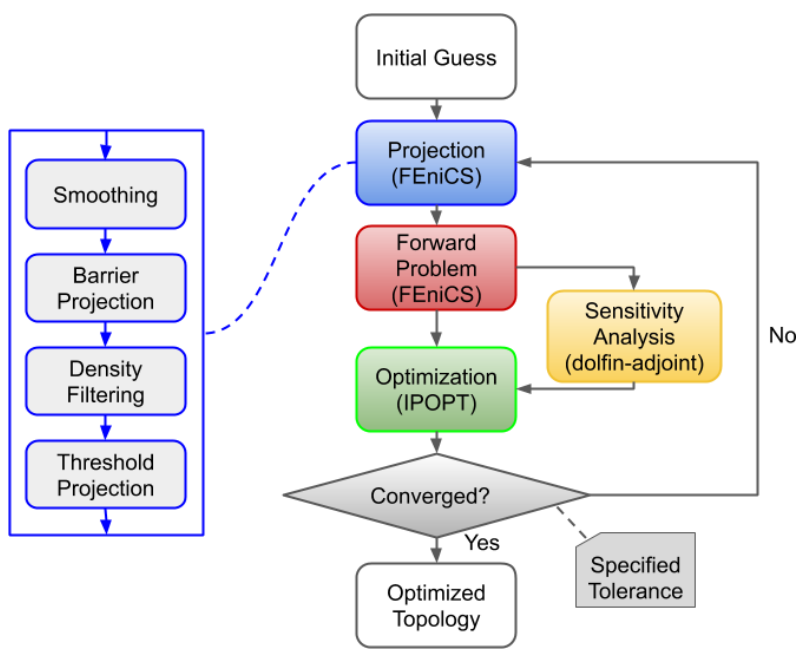

Figure 3.1 - Flowchart describing the algorithm used in this work. 


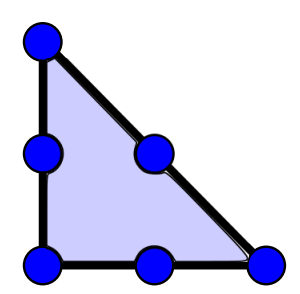

(a)

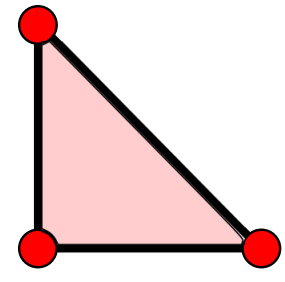

(b)

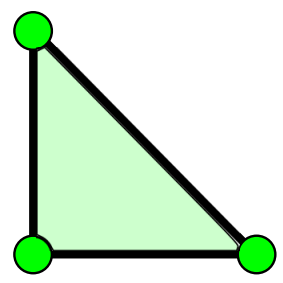

(c)

Figure 3.2 - Finite element discretization. (a) Displacement discretization; (b) Pressure discretization; (c) Design variable discretization.

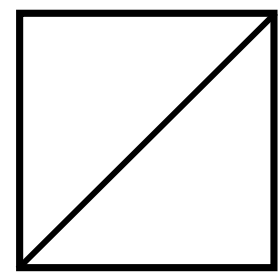

(a)

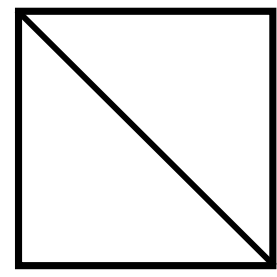

(b)

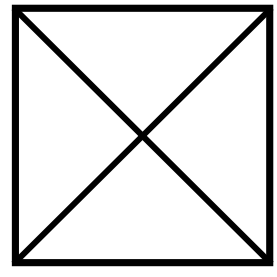

(c)

Figure 3.3 - FEniCS' finite element meshes. (a) Right mesh; (b) Left mesh; (c) Crossed mesh.

Programming Interface) are found in the book of Logg et al. (2012). Part of the API described by the book may be different depending on FEniCS version used.

The FEniCS framework supports triangular finite elements and several function spaces families, such as Lagrange, Discontinuous Lagrange, Hermite, Raviart-Thomas, and others. This work uses Lagrange function spaces of degree two for displacement and one for pressure and design variable field according to Figure 3.2.

The meshes supported by FEniCS are right, left, and crossed meshes which are illustrated by Figure 3.3. For the same element size and function space, right and left meshes have less elements and, therefore, less nodes than crossed mesh. Consequently, problems are solved faster on right and left meshes. However, there is a directional impact in topology optimization result that may be important depending on the problem being solved.

The sensitivities are calculated by the adjoint method using dolfin-adjoint package (FARRELL et al., 2013; FUNKE; FARRELL, 2013). The objective function derivatives are fully calculated by dolfin-adjoint and the volume constraint derivatives are calculated by the approach presented in Section 3.2.

The optimization routines use an interior-point optimization algorithm of IPOPT library (WäCHTER; BIEGLER, 2005). IPOPT is an open-source optimizer for large-scale nonlinear problems and it is suitable for solving compliant mechanisms design problems by topology optimization according to Rojas-Labanda and Stolpe (2015). Details about its implementation and API are given in Wächter (2009). 
The termination criteria used during optimization are desired convergence tolerance $\left(\epsilon_{t o l}\right)$ and maximum number of iterations $\left(n_{\max }\right)$. In IPOPT, the desired convergence is the optimality error of the rewritten barrier problem as described in Wächter and Biegler (2005). The maximum number of iterations limits the number of iterations to $n_{\max }$ if the convergence tolerance $\epsilon_{t o l}$ is not achieved yet. In this work, the maximum number of iterations is increased at each continuation step, so $n_{\max }$ is presented as a list. If the number of continuation steps is greater than the size of $n_{\max }$ list, the last value of list is used in subsequent iterations.

In Chapter 4, each design problem uses specific $\epsilon_{t o l}$ and $n_{\max }$. So, each design problem section $\left(4.1,4.2\right.$, and 4.3) presents its own $\epsilon_{t o l}$ and $n_{\max }$.

\subsection{Sensitivity Analysis}

The sensitivity analysis starts by considering the finite element discretization of all density fields involved in the projection of design variables to physical densities according to Equation 3.1 where $N_{\rho}$ is a vector of shape functions considered to be the same for all density fields.

$$
\begin{aligned}
& \rho_{e} \approx \boldsymbol{N}_{\rho} \boldsymbol{\rho}_{e} \\
& \bar{\rho}_{e} \approx \boldsymbol{N}_{\boldsymbol{\rho}} \overline{\boldsymbol{\rho}}_{e} \\
& \tilde{\rho}_{e} \approx \boldsymbol{N}_{\rho} \tilde{\boldsymbol{\rho}}_{e} \\
& \overline{\bar{\rho}}_{e} \approx \boldsymbol{N}_{\boldsymbol{\rho}} \overline{\bar{\rho}}_{e} \\
& \hat{\rho}_{e} \approx \boldsymbol{N}_{\rho} \hat{\boldsymbol{\rho}}_{e}
\end{aligned}
$$

As the physical densities are obtained by filtering and projecting the design variables, the sensitivities are calculated by applying the chain rule according to Equation 3.2. The design variables are the degrees of freedom of FEM approximation, which are presented in vector $\boldsymbol{\rho}$. Also, partial derivatives in bold $\boldsymbol{\partial} \boldsymbol{\rho}$ are derivatives with respect to degrees of freedoms $\boldsymbol{\rho}$. Equations 3.3 and 3.4 highlight the form of elements of Equation 3.2.

$$
\begin{gathered}
\frac{\partial J}{\partial \boldsymbol{\rho}}=\frac{\partial \overline{\boldsymbol{\rho}}}{\partial \boldsymbol{\rho}} \frac{\boldsymbol{\partial} \tilde{\boldsymbol{\rho}}}{\partial \bar{\rho}} \frac{\partial \overline{\bar{\rho}}}{\partial \tilde{\rho}} \frac{\partial \hat{\boldsymbol{\rho}}}{\partial \overline{\bar{\rho}}} \frac{\partial J}{\partial \hat{\rho}} \\
\frac{\partial J}{\partial \boldsymbol{\rho}}=\left[\begin{array}{lll}
\frac{\partial J}{\partial \rho_{1}} & \cdots & \frac{\partial J}{\partial \rho_{M}}
\end{array}\right]^{T} \\
\frac{\partial \bar{\rho}}{\partial \boldsymbol{\rho}}=\left[\begin{array}{ccc}
\frac{\partial \bar{\rho}_{1}}{\partial \rho_{1}} & \ldots & \frac{\partial \bar{\rho}_{M}}{\partial \rho_{1}} \\
\vdots & \ddots & \vdots \\
\frac{\partial \bar{\rho}_{M}}{\partial \rho_{1}} & \ldots & \frac{\partial \bar{\rho}_{M}}{\partial \rho_{M}}
\end{array}\right]
\end{gathered}
$$


The first term to be calculated is the derivative of $\bar{\rho}$ with respect to $\rho$. It is obtained by substituting the finite element discretization of Equations 3.1 and 3.5 into Equation 2.64 according to Equation 3.6. This equation is considered on the domain $\Omega_{e}$ of an element.

$$
\begin{gathered}
\nabla \rho_{e}=\nabla \boldsymbol{N}_{\boldsymbol{\rho}} \boldsymbol{\rho}_{\boldsymbol{e}}=\boldsymbol{B}_{\boldsymbol{\rho}} \boldsymbol{\rho}_{\boldsymbol{e}} \\
l^{2} \int_{\Omega_{e}}\left(\boldsymbol{B}_{\boldsymbol{\rho}} \overline{\boldsymbol{\rho}}_{\boldsymbol{e}}\right)^{T} \boldsymbol{B}_{\boldsymbol{\rho}} \boldsymbol{\delta} \boldsymbol{\rho}_{e} d \Omega+\int_{\Omega_{e}}\left(\boldsymbol{N}_{\boldsymbol{\rho}} \overline{\boldsymbol{\rho}}_{\boldsymbol{e}}\right) \boldsymbol{N}_{\boldsymbol{\rho}} \boldsymbol{\delta} \boldsymbol{\rho}_{\boldsymbol{e}} d \Omega=\int_{\Omega_{e}}\left(\boldsymbol{N}_{\boldsymbol{\rho}} \boldsymbol{\rho}_{\boldsymbol{e}}\right) \boldsymbol{N}_{\boldsymbol{\rho}} \boldsymbol{\delta} \boldsymbol{\rho}_{e} d \Omega
\end{gathered}
$$

It is possible to move the test function vector $\delta \rho_{e}$ out of integrals according to Equation 3.7 .

$$
\boldsymbol{\delta} \boldsymbol{\rho}_{e}^{\boldsymbol{T}}\left(\int_{\Omega_{e}} l^{2} \boldsymbol{B}_{\rho}^{\boldsymbol{T}} \boldsymbol{B}_{\rho} \overline{\boldsymbol{\rho}}_{e} d \Omega\right)+\boldsymbol{\delta} \boldsymbol{\rho}_{e}^{\boldsymbol{T}}\left(\int_{\Omega_{e}} \boldsymbol{N}_{\rho}^{\boldsymbol{T}} \boldsymbol{N}_{\boldsymbol{\rho}} \overline{\boldsymbol{\rho}}_{e} d \Omega\right)=\boldsymbol{\delta} \boldsymbol{\rho}_{e}^{\boldsymbol{T}}\left(\int_{\Omega_{e}} \boldsymbol{N}_{\rho}^{\boldsymbol{T}} \boldsymbol{N}_{\rho} \boldsymbol{\rho}_{e} d \Omega\right)
$$

Equation 3.7 has to hold for any arbitrary test function $\delta \boldsymbol{\rho}_{\boldsymbol{e}}$. So, Equation 3.8 is obtained.

$$
\left(l^{2} \int_{\Omega_{e}} \boldsymbol{B}_{\rho}^{\boldsymbol{T}} \boldsymbol{B}_{\rho}+\boldsymbol{N}_{\rho}^{\boldsymbol{T}} \boldsymbol{N}_{\boldsymbol{\rho}} d \Omega\right) \overline{\boldsymbol{\rho}}_{\boldsymbol{e}}=\left(\int_{\Omega_{e}} \boldsymbol{N}_{\rho}^{\boldsymbol{T}} \boldsymbol{N}_{\boldsymbol{\rho}} d \Omega\right) \boldsymbol{\rho}_{\boldsymbol{e}}
$$

By differentiating Equation 3.8 with respect to $\boldsymbol{\rho}_{\boldsymbol{e}}$, Equation 3.9 is obtained where matrices $\boldsymbol{A}_{\boldsymbol{l}}$ and $\boldsymbol{B}$ are given by Equation 3.10. The operator $\sum_{e}$ is the summation over elements (assembly operator).

$$
\begin{gathered}
\boldsymbol{A}_{l} \frac{\partial \overline{\boldsymbol{\rho}}}{\partial \boldsymbol{\rho}}=\boldsymbol{B} \\
\boldsymbol{A}_{l}=\sum_{e}\left(l^{2} \int_{\Omega_{e}} \boldsymbol{B}_{\boldsymbol{\rho}}^{\boldsymbol{T}} \boldsymbol{B}_{\rho}+\boldsymbol{N}_{\boldsymbol{\rho}}^{\boldsymbol{T}} \boldsymbol{N}_{\boldsymbol{\rho}} d \Omega\right) \\
\boldsymbol{B}=\sum_{e}\left(\int_{\Omega_{e}} \boldsymbol{N}_{\boldsymbol{\rho}}^{\boldsymbol{T}} \boldsymbol{N}_{\boldsymbol{\rho}} d \Omega\right)
\end{gathered}
$$

The same procedure to obtain Equation 3.9 is used to calculate $\frac{\partial \overline{\bar{\rho}}}{\partial \tilde{\rho}}$ if $l^{2}$ is replaced by $r^{2}$. In this case, Equation 3.11 is obtained.

$$
\begin{gathered}
\boldsymbol{A}_{r} \frac{\partial \overline{\bar{\rho}}}{\partial \tilde{\rho}}=B \\
\boldsymbol{A}_{r}=\left(r^{2} \int_{\Omega_{e}} \boldsymbol{B}_{\rho}^{\boldsymbol{T}} \boldsymbol{B}_{\rho}+\boldsymbol{N}_{\rho}^{\boldsymbol{T}} \boldsymbol{N}_{\boldsymbol{\rho}} d \Omega\right)
\end{gathered}
$$


The derivatives of Equations 2.65 and 2.67 are given by Equations 3.13 and 3.14, respectively. The matrices are diagonal because each degree of freedom is projected on itself (there is no cross influence between variables). Besides reducing the computations, the diagonal matrices are interesting from a computational point of view because they save memory.

$$
\begin{gathered}
\frac{\partial \tilde{\rho}_{i}}{\partial \bar{\rho}_{j}}= \begin{cases}-\alpha \bar{\rho}_{j}^{\alpha-1}, & \text { if } i=j \\
0, & \text { if } i \neq j\end{cases} \\
\frac{\partial \hat{\rho}_{i}}{\partial \overline{\bar{\rho}}_{j}}= \begin{cases}\frac{\beta\left(1-\tanh ^{2}\left(\beta\left(\overline{\bar{\rho}}_{j}-\eta\right)\right)\right)}{\tanh (\beta \eta)+\tanh (\beta(1-\eta))} & , \text { if } i=j \\
0 & , \text { if } i \neq j\end{cases}
\end{gathered}
$$

By multiplying Equation 3.2 by $\boldsymbol{A}_{\boldsymbol{l}}$, it is possible to use Equation 3.9 for replacing $\boldsymbol{\partial} \overline{\boldsymbol{\rho}} / \boldsymbol{\partial} \boldsymbol{\rho}$. With this approach, the problem of finding $\partial J / \partial \boldsymbol{\rho}$ becomes the solution of a linear system of equations given by Equation 3.15.

$$
\begin{aligned}
A_{l} \frac{\partial J}{\partial \rho} & =A_{l} \frac{\partial \bar{\rho}}{\partial \rho} \frac{\partial \tilde{\rho}}{\partial \bar{\rho}} \frac{\partial \overline{\bar{\rho}}}{\partial \tilde{\rho}} \frac{\partial \hat{\rho}}{\partial \overline{\bar{\rho}}} \frac{\partial J}{\partial \hat{\rho}} \\
& =B \frac{\partial \tilde{\rho}}{\partial \bar{\rho}} \frac{\partial \overline{\bar{\rho}}}{\partial \tilde{\rho}} \frac{\partial \hat{\rho}}{\partial \overline{\bar{\rho}}} \frac{\partial J}{\partial \hat{\rho}} \\
& =b
\end{aligned}
$$

The derivative of the objective function $J$ with respect to the degree of freedom $\hat{\rho}_{i}$ of the physical density is given by Equation 3.16.

$$
\frac{\partial J}{\partial \hat{\rho}_{i}}=\int_{\Gamma_{\text {out }}} \boldsymbol{l}^{\boldsymbol{T}} \frac{\partial \boldsymbol{u}}{\partial \hat{\rho}_{i}} d \Gamma
$$

To apply the adjoint method to calculate this derivative, the vector $\boldsymbol{l}$ is extended with zeros $\left(\boldsymbol{l}_{\text {ext }}\right)$ for each derivative of pressure with respect to the pseudo densities (Equation 3.17).

$$
\frac{\partial J}{\partial \hat{\rho}_{i}}=\int_{\Gamma_{\text {out }}} \boldsymbol{l}_{\text {ext }}^{\boldsymbol{T}}\left\{\begin{array}{c}
\frac{\partial \boldsymbol{u}}{\partial \hat{\rho}_{i}} \\
\frac{\partial \boldsymbol{p}}{\partial \hat{\rho}_{i}}
\end{array}\right\} d \Gamma
$$

Then, Equation 2.52 is differentiated with respect to $\hat{\rho}_{i}$. This result is used to obtain the adjoint equation (Equation 3.19) whose solution $\boldsymbol{\lambda}$ help the evaluation of sensitivities according 
to Equation 3.20.

$$
\begin{aligned}
&\left\{\begin{array}{c}
\frac{\partial \boldsymbol{u}}{\partial \hat{\rho}_{i}} \\
\frac{\partial \boldsymbol{p}}{\partial \hat{\rho}_{i}}
\end{array}\right\}=-\left[\begin{array}{cc}
\boldsymbol{K} & \boldsymbol{C} \\
\boldsymbol{C}^{T} & \boldsymbol{D}
\end{array}\right]^{-1}\left[\begin{array}{cc}
\frac{\partial \boldsymbol{K}}{\partial \hat{\rho}_{i}} & \frac{\partial \boldsymbol{C}}{\partial \hat{\rho}_{i}} \\
\frac{\partial \boldsymbol{C}^{T}}{\partial \hat{\rho}_{i}} & \frac{\partial \boldsymbol{D}}{\partial \hat{\rho}_{i}}
\end{array}\right]\left\{\begin{array}{l}
\boldsymbol{u} \\
\boldsymbol{p}
\end{array}\right\} \\
& {\left[\begin{array}{cc}
\boldsymbol{K} & \boldsymbol{C} \\
\boldsymbol{C}^{T} & \boldsymbol{D}
\end{array}\right]^{T} \boldsymbol{\lambda}=-\boldsymbol{l}_{\boldsymbol{e x t}} } \\
& \frac{\partial J}{\partial \hat{\rho}_{i}}=\int_{\Gamma_{\text {out }}} \boldsymbol{\lambda}^{\boldsymbol{T}}\left[\begin{array}{cc}
\frac{\partial \boldsymbol{K}}{\partial \hat{\rho}_{i}} & \frac{\partial \boldsymbol{C}}{\partial \hat{\rho}_{i}} \\
\frac{\partial \boldsymbol{C}^{T}}{\partial \hat{\rho}_{i}} & \frac{\partial \boldsymbol{D}}{\partial \hat{\rho}_{i}}
\end{array}\right]\left\{\begin{array}{l}
\boldsymbol{u} \\
\boldsymbol{p}
\end{array}\right\} d \Gamma
\end{aligned}
$$

The same method is used to calculate the derivative of the volume constraint with respect to the design variables. The derivative of $g$ with respect to the pseudo densities is given by Equation 3.21.

$$
\frac{\partial g}{\partial \hat{\rho}_{i}}=\int_{\Omega} \phi_{i} d \Omega
$$

\subsection{Inverter Mechanism Design in FeniCS}

This section explains how to use FEniCS and dolfin-adjoint to implement the design of an inverter compliant mechanism by topology optimization. Only the most relevant parts of the code are explained while trivial parts such as variable definitions are skipped. The complete code is presented in Appendix D.4.

The code starts by importing two modules: fenics (FEniCS module) and fenics_adjoint (dolfin-adjoint module) according to Source Code 3.1. The dolfin-adjoint module must be imported after the FEniCS module, because it overrides some FEniCS functions.

Source Code 3.1 - Importing FEniCS and dolfin-adjoint modules.

1 from fenics import *

2 from fenics_adjoint import *

Then, it proceeds with an attempt to import the wrapper module to IPOPT library: pyipopt. If the import fails, an exception is raised. This step is presented in Source Code 3.2.

Source Code 3.2 - Importing pyipopt wrapper module. 


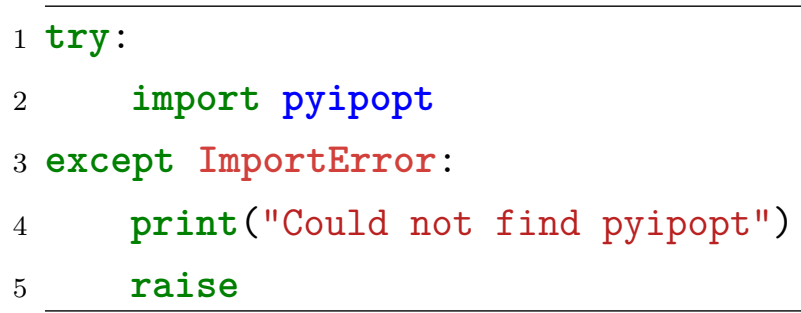

After importing pyipopt, the code declares relevant constants of the problem. The next important snippet of code is the declaration of the mesh to be used (Source Code 3.3). A rectangular mesh of width $L_{X}$ and height $L_{Y}$ is declared with $n_{x}$ divisions in horizontal direction and $n_{y}$ divisions in vertical direction. This mesh is divided in triangles arranged according to the crossed mesh of Figure 3.3.

Source Code 3.3 - Mesh declaration.

1 mesh $=$ RectangleMesh (Point $(0,0)$, Point (L_X, L_Y $), n_{-} \mathrm{x}, \mathrm{n}_{-} \mathrm{y}$, "crossed")

The next step is to define the function spaces to be used. To declare a function space, it is necessary to select a mesh, a family of functions and the degree of the functions. For the inverter mechanism problem, the displacements $\boldsymbol{u}$ are discretized by Continuous-Galerkin functions of degree 2 (line 1 of Source Code 3.4). The design variable field $\rho$ (pseudo-density field) is discretized by Continuous-Galerkin of degree 1 (line 2 of Source Code 3.4).

Source Code 3.4 - Function spaces for inverter actuator design.

$1 \longdiv { \mathrm { V } } =$ VectorFunctionSpace(mesh, "CG", 2)

$2 \mathrm{~W}=$ FunctionSpace (mesh, "CG", 1)

Then, some sub-domains are defined and created to specify the boundary conditions (Source Code 3.5). The first sub-domain defined is the upper edge which represents a symmetry edge to enable the reduction of optimization time by running the algorithm on half of the actuator. The second sub-domain is the support defined on the left corner of lower edge. The displacements are fixed to be zero at the support sub-domain.

Source Code 3.5 - Boundary conditions for inverter actuator design.

class Symmetry(SubDomain): 


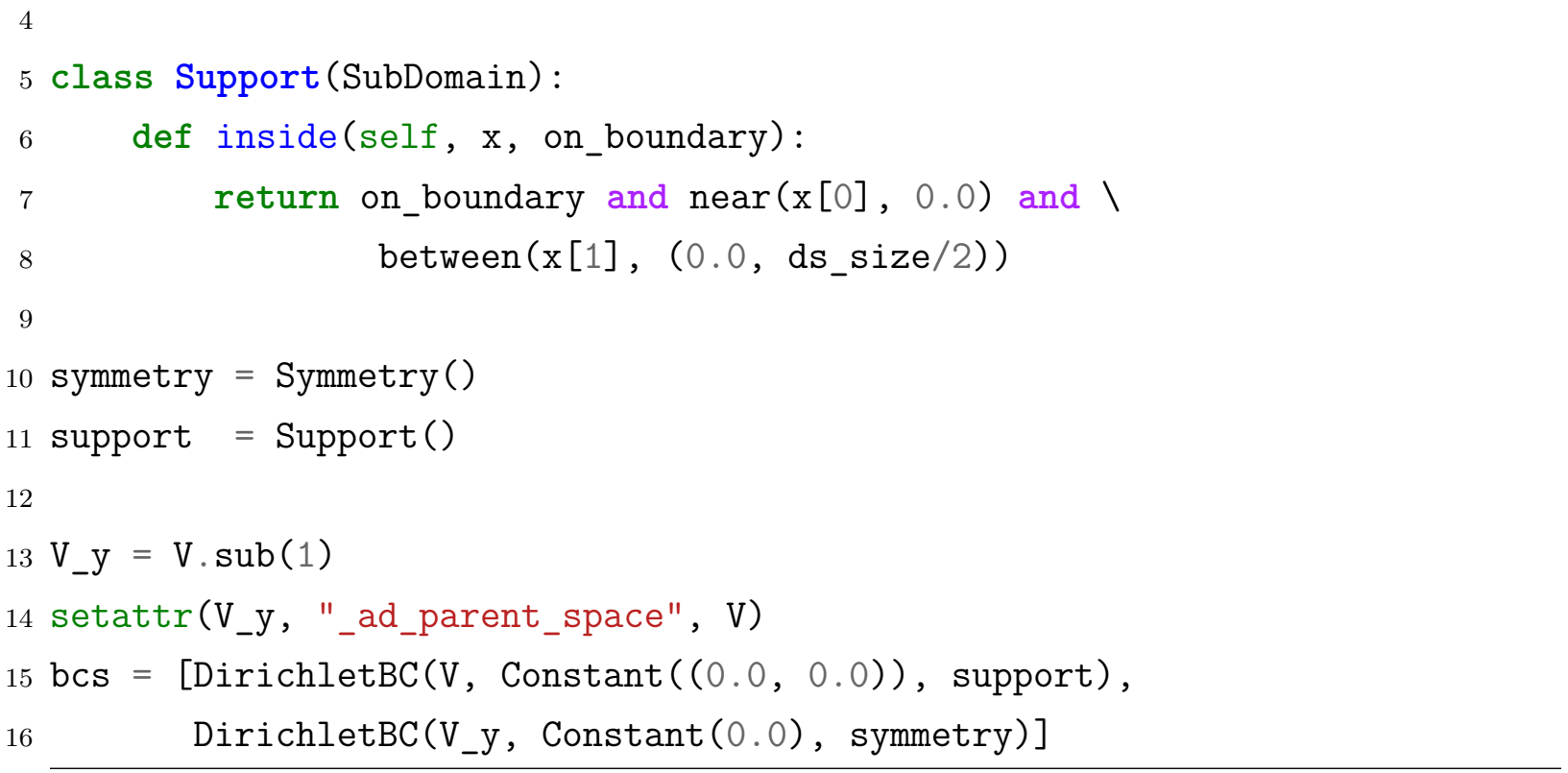

The input and output ports of the compliant mechanism are defined from lines 1 to 12 of Source Code 3.6. These objects are used to mark the parts of the boundary which are input and output ports according to lines 14 to 19. The input force and the input spring are placed in the input port. The output spring is placed in the output port and the objective function is calculated at the output port.

Source Code 3.6 - Input and output port definitions.

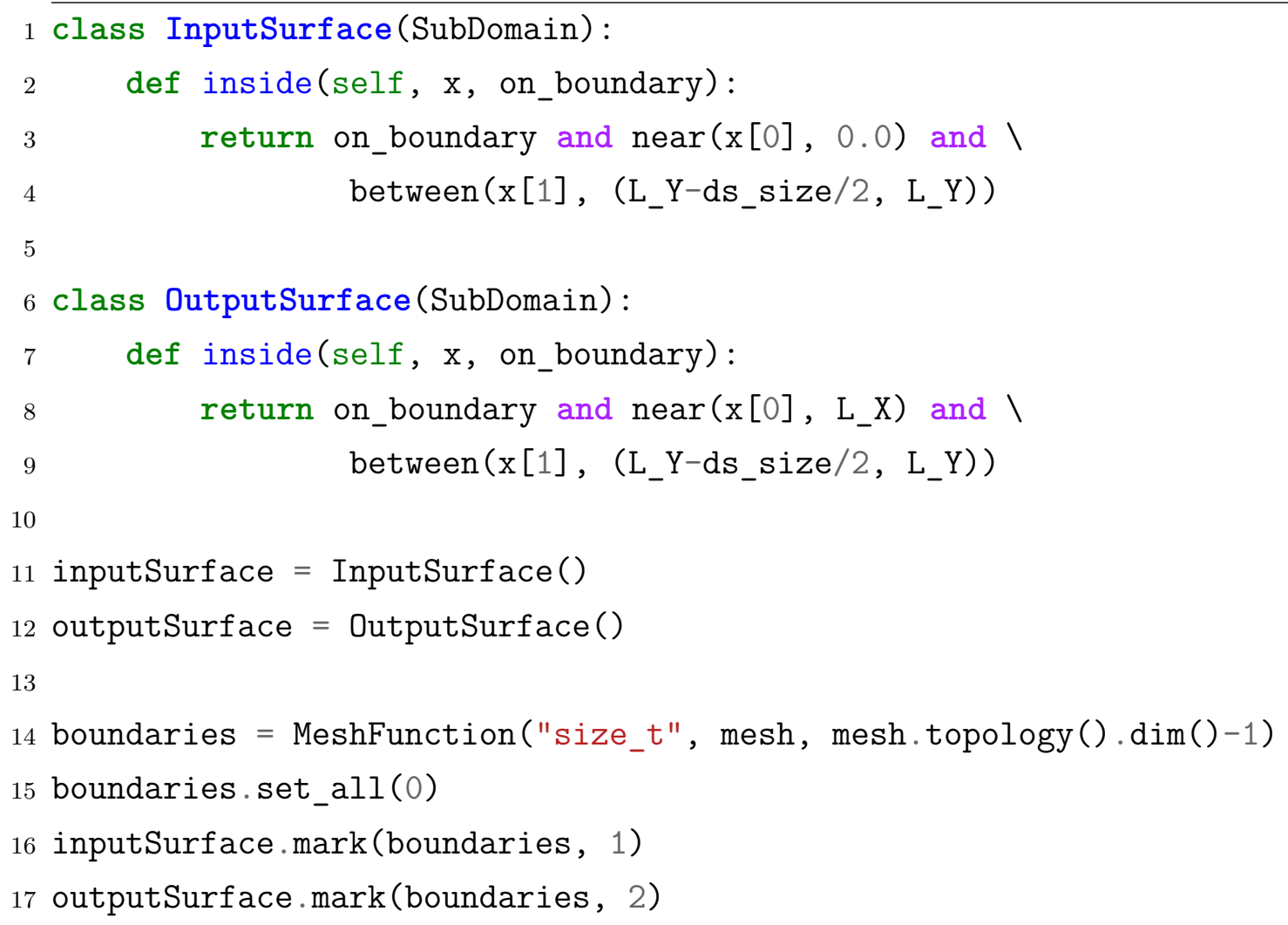


$19 \mathrm{ds}=\mathrm{ds}($ subdomain_data $=$ boundaries $)$

The forward problem of Equation 3.22 is solved by Source Code 3.7 which starts with the definition of the displacement trial function $\boldsymbol{u}$ (line 2) and the displacement test function $\boldsymbol{v}$ $=\boldsymbol{\delta} \boldsymbol{u}$ (line 3). Then, the SIMP interpolation of the Young's modulus is calculated at line 5 and its value is used to compute the Lamé's parameters $\lambda$ and $\mu$ in lines 6 and 7 . The static equilibrium in the variational form of Equation 3.22 is defined from lines 9 to 13 . The FEM matrix are assembled in line 15 and the FEM linear system is solved in line 17.

$$
\begin{array}{r}
2 \mu \int_{\Omega} \operatorname{Sym}(\nabla \boldsymbol{u}): \operatorname{Sym}(\nabla \boldsymbol{\delta} \boldsymbol{u}) d \Omega+\lambda \int_{\Omega}(\nabla \cdot \boldsymbol{u})(\nabla \cdot \boldsymbol{\delta} \boldsymbol{u}) d \Omega \\
+\int_{\Gamma_{\text {in }}} k_{\text {in }}\left(\boldsymbol{l}_{\text {in }} \cdot \boldsymbol{u}\right)\left(\boldsymbol{l}_{\text {in }} \cdot \boldsymbol{\delta} \boldsymbol{u}\right) d \Gamma \\
+\int_{\Gamma_{\text {out }}} k_{\text {out }}\left(\boldsymbol{l}_{\text {out }} \cdot \boldsymbol{u}\right)\left(\boldsymbol{l}_{\text {out }} \cdot \boldsymbol{\delta} \boldsymbol{u}\right) d \Gamma \\
=\int_{\Gamma_{\text {in }}} \boldsymbol{t} \cdot \boldsymbol{\delta} \boldsymbol{u} d \Gamma
\end{array}
$$

Source Code 3.7 - Forward problem of inverter actuator design.

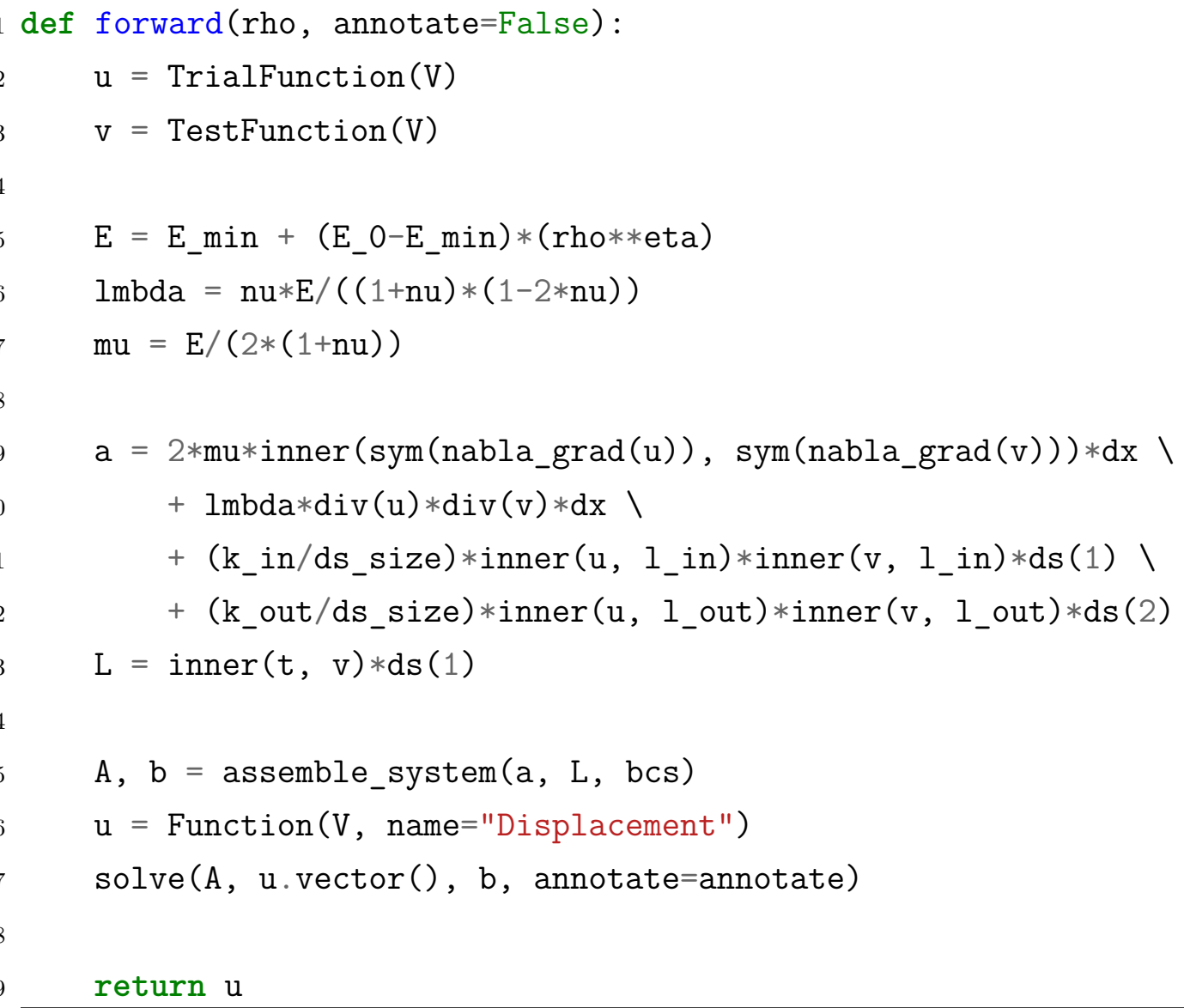


The volume constraint of Equation 3.23 and the Jacobian of Equation 3.24 are defined in Source Code 3.8. The constructor is declared from lines 2 to 5 . The value of the constraint $g$ is calculated by the method "function" defined from lines 7 to 14 . The Jacobian is calculated in lines 16 and 17 . The number of equations is defined in method "length" in lines 22 and 23.

$$
\begin{gathered}
g(\boldsymbol{\rho})=V^{*}-\int_{\Omega} \rho(\boldsymbol{x}) d \Omega=V^{*}-\int_{\Omega} \sum_{i=1}^{M} \rho_{i} \phi_{i}(\boldsymbol{x}) d \Omega \\
\frac{\boldsymbol{\partial} \boldsymbol{g}}{\boldsymbol{\partial} \boldsymbol{\rho}}=-\left[\begin{array}{c}
\int_{\Omega} \phi_{1}(\boldsymbol{x}) d \Omega \\
\vdots \\
\int_{\Omega} \phi_{M}(\boldsymbol{x}) d \Omega
\end{array}\right]
\end{gathered}
$$

Source Code 3.8 - Volume constraint definition.

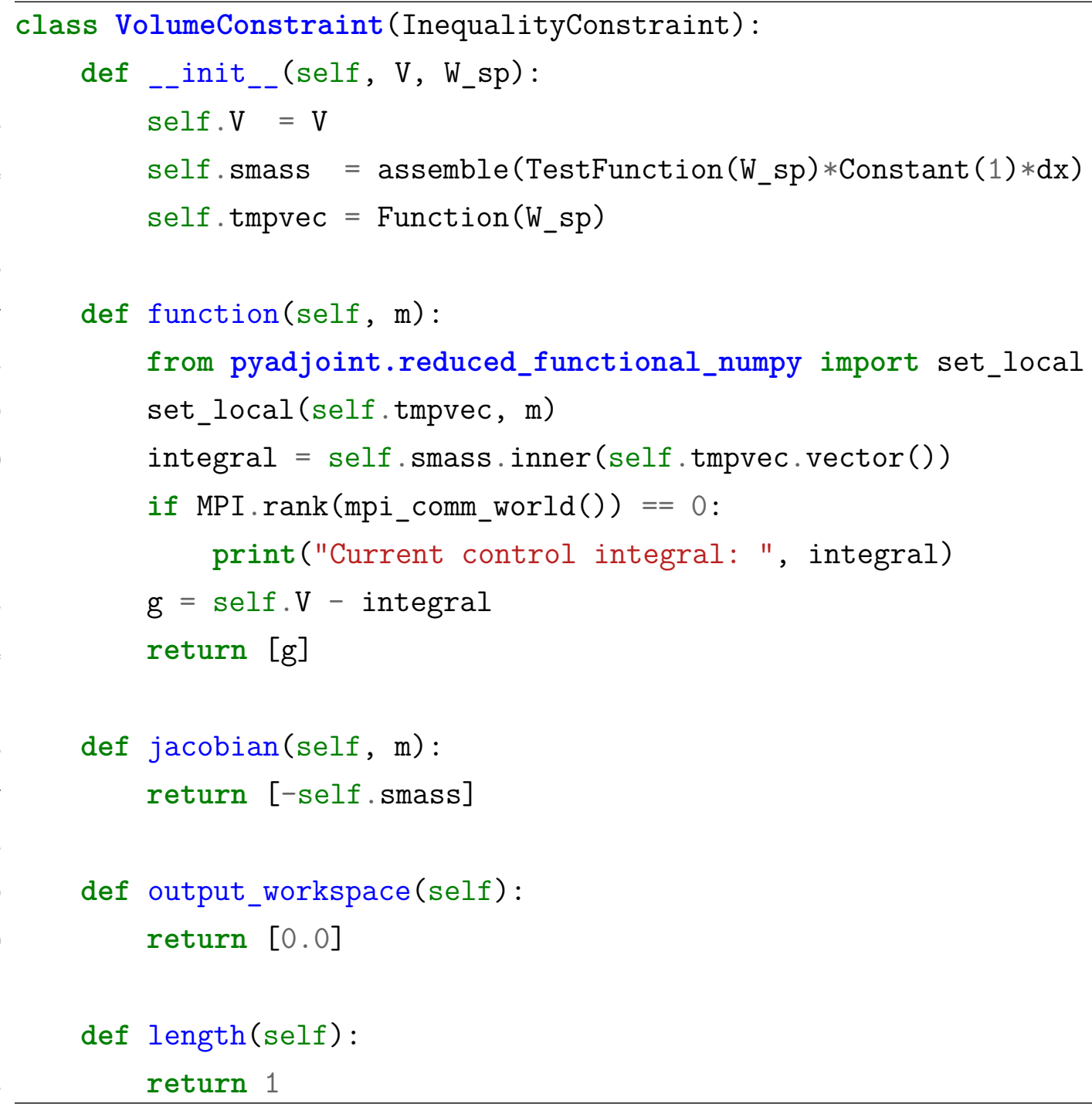

The final step is the solution of the optimization problem according to Source Code 3.9. 
First, the initial guess is declared in line 2 and it is used to solve the forward problem in line 3. The forward problem is annotated to indicate to dolfin-adjoint that the forward problem must be solved at each optimization iteration. Then, the functional to be minimized (negative output displacement) is declared in lines 5 and 6 . This is equivalent to maximize the output displacement. From lines 8 to 10 , the bound constraint from $\mathrm{lb}$ to ub and a volume constraint of $25 \%$ of the area of the design space are defined. In line 19 , the optimization problem is solved with the parameters from lines 15 to 17 . Finally, the solution is exported in lines 21 and 22 for visualization in external softwares.

Source Code 3.9 - Solution of optimization problem.

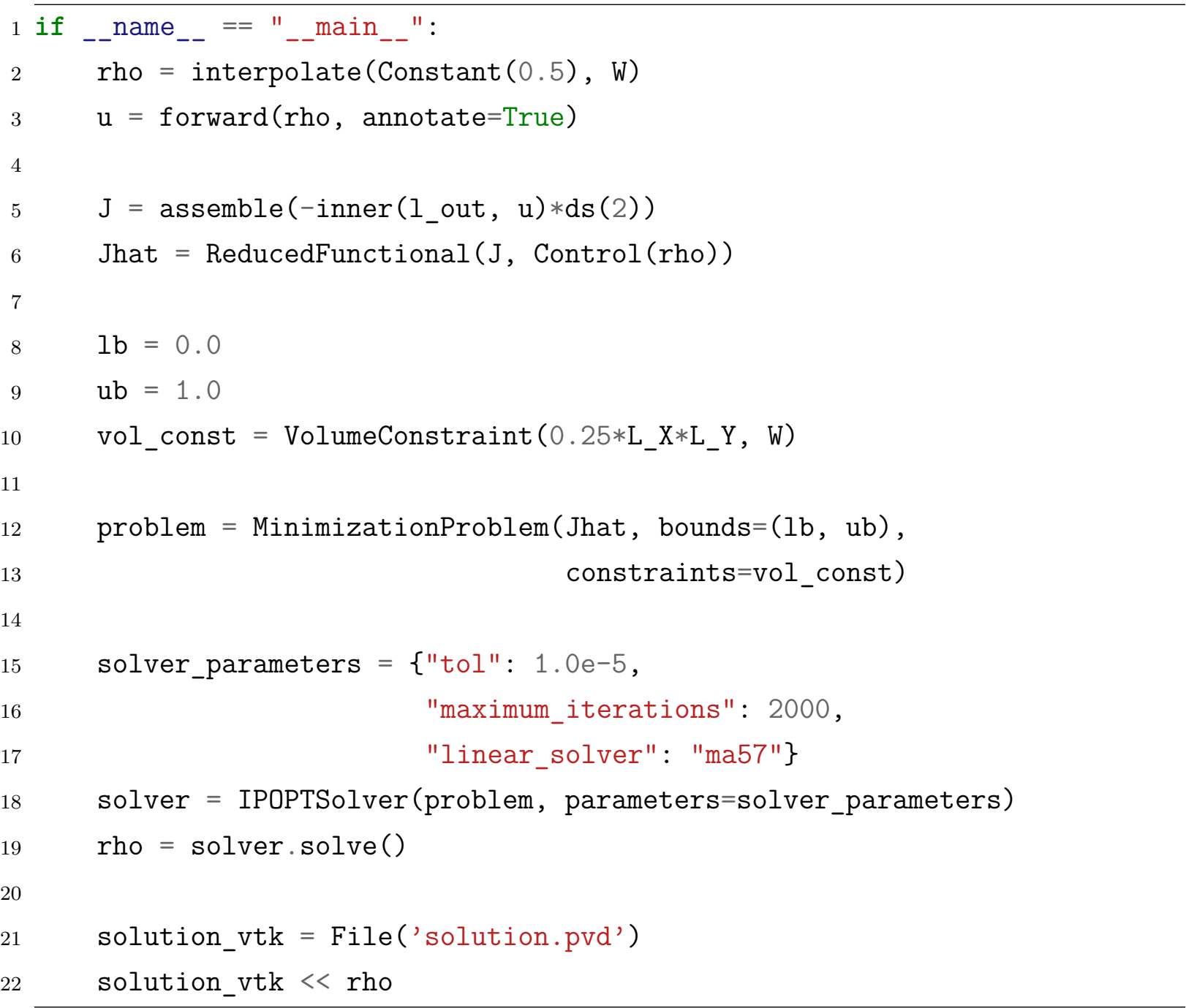

The execution of the complete code presented in Annex D.4 produces the result presented in Figure 3.4. The topology of Figure 3.4 is in accordance with literature results such as the result presented by Pedersen, Buhl and Sigmund (2001). 


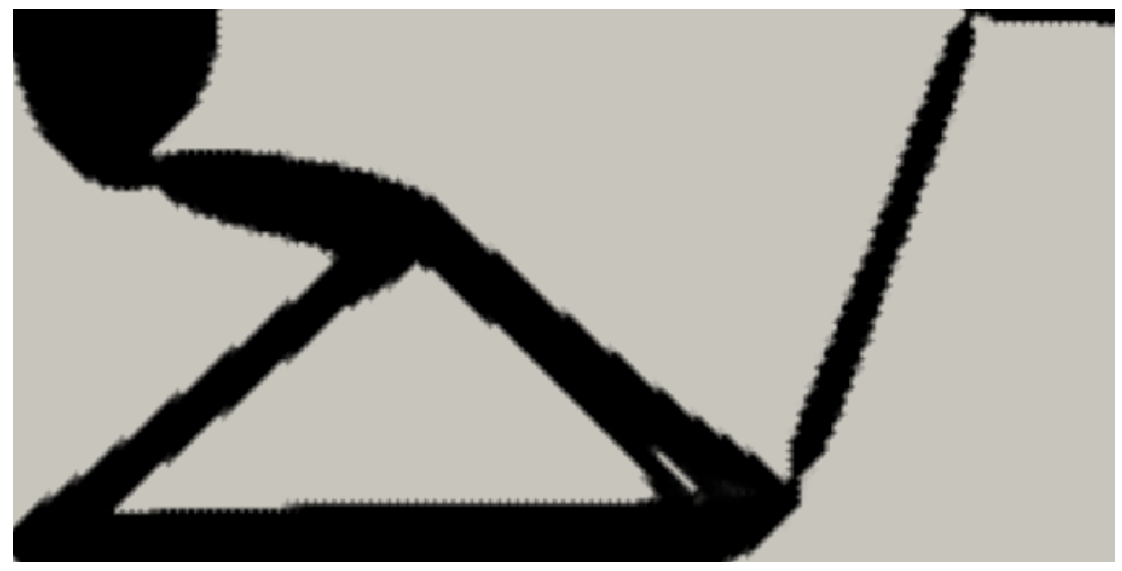

Figure 3.4 - Inverter actuator result obtained with the code presented in Annex D.4. 


\section{RESULTS}

This chapter presents the results obtained for different design problems. At each section the optimization is first done at the interior surface, then on the interior and exterior surfaces, and finally by using the proposed barrier projection technique. The rationale for this approach is to walk towards the most general optimization routine while avoiding open designs. With this progression, it is possible to better evaluate the output of the software because the objective function has to enhance as the formulation becomes more general. Each section ends with the comparison of results and a nonlinear analysis of the result obtained with barrier projection according to Appendix B.

Three design problems are studied. The first is the design of a bending actuator which is the most common type of soft actuator found in literature (e. g., Suzumori (1996), Galloway et al. (2013), Mosadegh et al. (2014)). The bending movement is obtained by the placement of the compliant mechanism output port at the tip of the lower edge of the actuator according to Figure 4.1a. The second problem is the design of an actuator that stretches along its length producing linear motion. It will be called henceforth the linear problem and its design domain is presented in Figure 4.1b. The third problem is also optimized on the design domain of Figure $4.1 \mathrm{~b}$, but the output port direction is inverted in horizontal direction. This problem will be called the inverter problem. The design of inverter compliant mechanisms is usual as benchmark for compliant mechanism formulations, so this design problem is also studied in this work to check robustness of barrier projection technique.

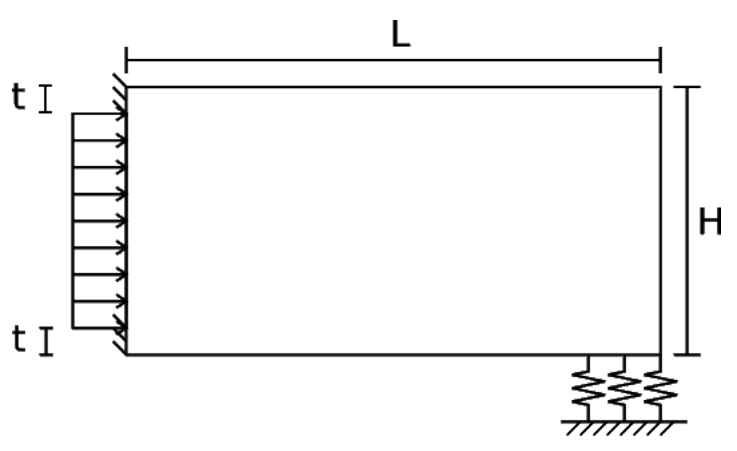

(a)

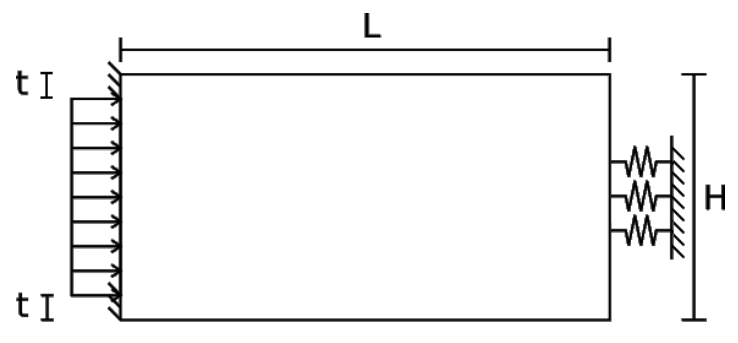

(b)

Figure 4.1 - Design domain for the problems studied in this work. (a) Bending design domain; (b) Linear and Inverter design domains.

The design domains from Figure 4.1 present more than one spring at each output port surface $\Gamma_{\text {out }}$ to indicate that the elastic force is distributed and not placed on a single FEM node in this work. This is done because the input of FEniCS are variational forms of the physical equations and the spring force can only be described as a distributed force on variational forms. 
Table 4.1 - Material properties used for simulations.

\begin{tabular}{lcc}
\hline Material & Shear Modulus (G) & Bulk Modulus (K) \\
\hline Solid & $3.6 \mathrm{MPa}$ & $25 \mathrm{MPa}$ \\
Air & $1 \mu \mathrm{Pa}$ & $101 \mathrm{kPa}$ \\
Water & $1 \mu \mathrm{Pa}$ & $2.15 \mathrm{GPa}$ \\
\hline
\end{tabular}

In fact, this abstraction is closer to reality, because there are no nodal forces in physical world. In the end, the distributed forces are converted to nodal forces by the FEniCS framework to solve the problem numerically.

As the objective of this study is to provide design tools for soft actuators fabricated from elastomeric materials, the solid material properties used are Young's modulus $E_{S}=10 \mathrm{MPa}$ and Poisson's ratio $\nu_{S}=0.4$ which are values found in some elastomers ( $L A I$; RUBIN; KREMPL, 2009). Some optimizations are also carried with $\nu_{S}=0.49$ to simulate nearly incompressibility as elastomers present incompressibility. Physical values are chosen to verify if the topology optimization approach developed converges when using real world parameters.

For mixed model, air material properties are bulk modulus $K_{F}=101 \mathrm{kPa}$ (Appendix D.3) and shear modulus $G_{F}=1 \mu \mathrm{Pa}$. Although fluids do not sustain shear stress $\left(G_{F}=0\right)$, a small value of $1 \mu \mathrm{Pa}$ is used to avoid singularities. The last section considers water material properties $\left(K_{F}=2.15 \mathrm{GPa}\right.$ according to Song (2018)) to evaluate the design of hydraulic actuators. These values are summarized in Table 4.1.

The finite elements used are right triangular Lagrange elements of size $e l_{\text {size }}=2.5 \mathrm{~mm}$.

\subsection{Bending Actuator}

Soft bending actuators are designed to curve in one or more directions when pressurized. In this work, bending actuators that curve in one direction are designed by placing the output port at the tip of design domain in attempt to make the actuator move in that direction by bending.

The actuator is designed on a rectangular domain and each optimization approach presents different fixed regions according to Figure 4.2. These fixed regions have constant design variables values during optimization. The input pressure is applied at left edge as a Dirichlet boundary condition to pressure variable. The actuator is fixed at upper and lower left edge. A distributed spring of stiffness $k_{\text {out }}$ is placed at the lower right edge to represent the work piece. Its direction and orientation are considered to be $\boldsymbol{l}_{\text {out }}=[0,-1]^{T}$ for all bending actuator synthesis.

The Interior Surface problem (Figure 4.2a) is optimized in a design domain of length $L_{D}=$ 


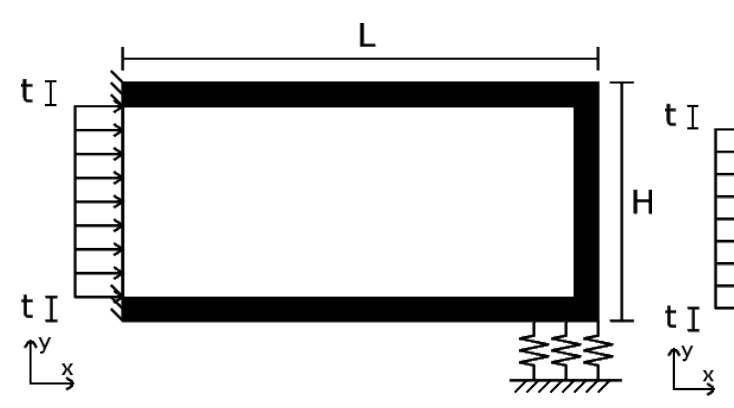

(a)

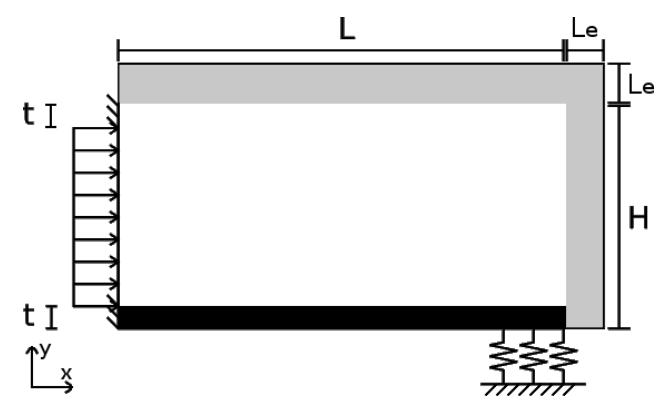

(c)

Figure 4.2 - Design domain with constraints for bending actuator problem. (a) Interior; (b) Interior/Exterior; (c) Barrier.

$L$ and height $H_{D}=H$ while the Interior/Exterior Surfaces (Figure 4.2b) and Barrier (Figure 4.2c) problems are optimized on a extended design domain of length $L_{D}=L+L_{E}$ and height $H_{D}=H+L_{E}$. The length parameters used for bending actuator are $L=180 \mathrm{~mm}, H=80$ $\mathrm{mm}$, and $L_{E}=20 \mathrm{~mm}$.

A fixed solid enclosure of thickness $t$ is placed on Interior Surface (Figure 4.2a) and Interior/Exterior Surfaces (Figure 4.2b) problems to keep the actuator closed during optimization. The enclosure has thickness $t=10 \mathrm{~mm}$. For the Barrier problem (Figure 4.2c), the extended region is fixed as exterior fluid, but there is no fixed solid enclosure.

The allowed material volume for all approaches is $V^{*}=V_{\text {frac }} L H$ (by considering unit depth) in order to obtain designs with the same mass even with the extension of the domain for Interior/Exterior Surfaces and Barrier problems. By using the same volume constraint the comparison of results is fairer. The $V_{\text {frac }}$ used is $50 \%$.

The termination criteria for bending actuator problem is presented in Equation 4.1.

$$
\begin{aligned}
& \epsilon_{\text {tol }}=10^{-4} \\
& n_{\text {max }}=\left[\begin{array}{llllllll}
25 & 25 & 25 & 25 & 50 & 150 & 200 & 500
\end{array}\right]^{T}
\end{aligned}
$$


Table 4.2 - Motivation to impose closed constraints or to introduce barrier projection. The bending actuator problem is solved considering different constraints according to table's header. The initial guess is the closed topology presented in Figure 4.2a for all examples. (a) Column showing results obtained for free optimization; (b) Column showing results obtained for lower edge constraint; (c) Column showing results obtained for closed constraint.

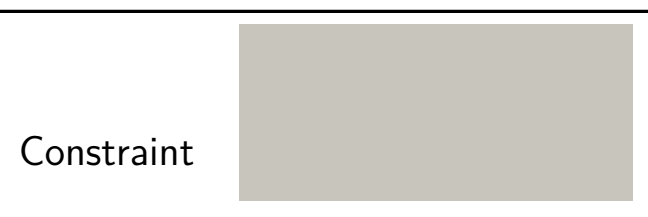

(a) Free optimization

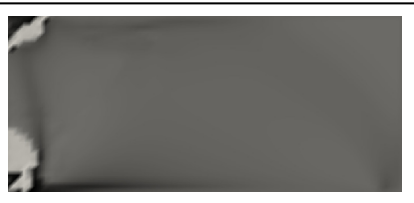

One-field

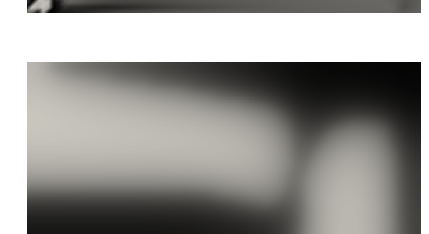

Two-field
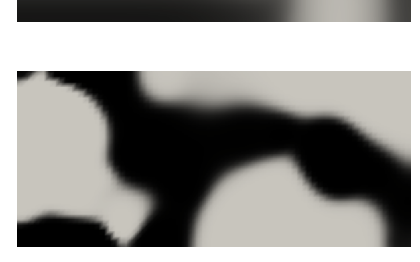

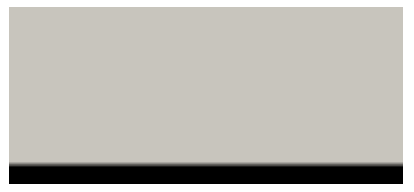

(b) Lower edge constraint
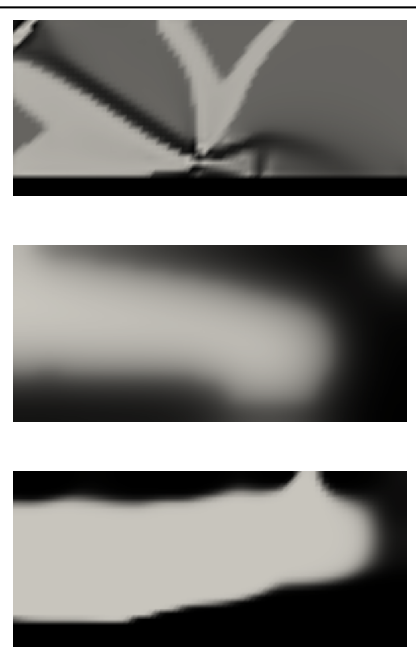

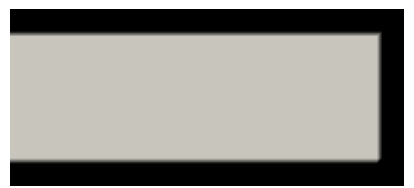

(c) Closed constraint
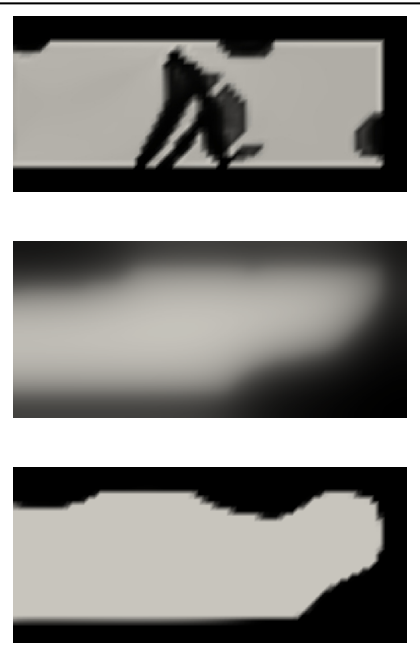

\subsubsection{Motivation}

The motivation to place a closed constraint or to introduce barrier projection is verified when trying to solve the problem without these approaches. Table 4.2 presents the results of solving the bending actuator problem stated in this section (Figure 4.2) by three different algorithms of topology optimization.

The first algorithm is an one-field topology optimization routine in which the design variable $\rho$ is the physical density variable. The second approach is a two-field topology optimization algorithm in which the filtered variable $\bar{\rho}$ (Equation 2.57 ) is the physical density. The third approach is a three-field topology optimization algorithm in which the projected variable $\hat{\rho}$ (Equation 2.58) is the physical density variable. These algorithms are described in detail by (SIGMUND; MAUTE, 2013).

Table 4.2 header shows which constraint is used for the results of each column. The initial guess is always a closed topology according to Figure 4.2a. The results in first row are for one-field algorithm, the results in second row are for two-field algorithm, and the results in third row are for three-field algorithm. 
According to the first (free optimization) and second (lower edge constraint) columns of Table 4.2, there is no guarantee of obtaining a closed actuator when using traditional approach to topology optimization without any enclosure constraint.

The third column of Table 4.2 shows that the use of density filter (Equation 2.57) and threshold projection (Equation 2.58) produces the better result. The density filter enhance convergence and the threshold projection eliminates the grey transitions regions introduced by the density filter. Therefore, it is advisable to use density filter and threshold projection when designing pressure driven compliant mechanisms to obtain good convergence and results free from grey physical densities that are of no interest.

\subsubsection{Interior Surface}

The Interior Surface optimization result is presented in Figure 4.3a. The objective function for the obtained topology is $J(\boldsymbol{u})=4.9 \mathrm{~mm}^{2}$. The visual inspection of the result indicates an almost black and white solution and the discreteness level of $M_{n d}=2.5 \%$ confirms the visual interpretation.

The deformed configuration is presented in Figure 4.3b with a red contour marking the undeformed configuration. It is possible to see that output port moves in direction $\boldsymbol{l}_{\text {out }}=[0,-1]^{T}$. The displacement is magnified 10 times to help visualization.

Figure $4.3 c$ presents convergence curves of objective function $(J(\boldsymbol{u}))$ and discreteness $\left(M_{n d}\right)$. The objective function curve is plotted as a solid blue line while the discreteness curve is plotted as a dashed red line. The steps at convergence graphs are associated to continuation of $\beta$ parameter. As $\beta$ increases between continuation iterations, regions of intermediate density change their physical density abruptly making the output displacement and discreteness change abruptly too.

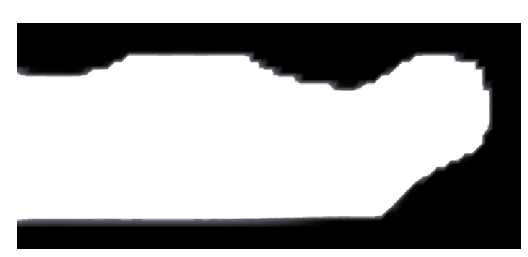

(a)

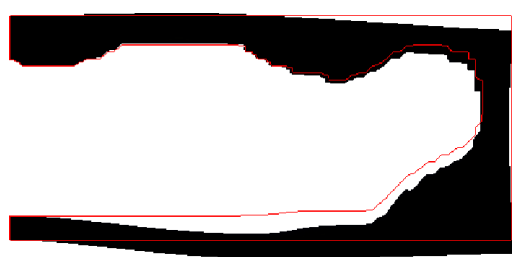

(b)

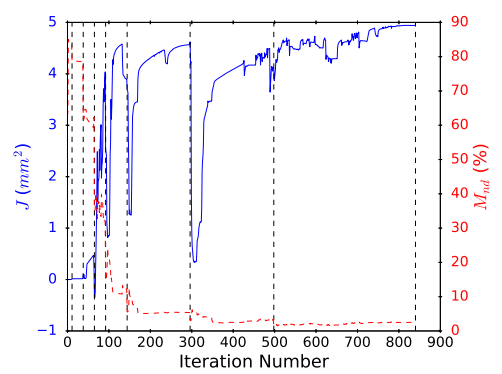

(c)

Figure 4.3 - Result of interior surfaces optimization for bending actuator problem. (a) Optimized topology; (b) Displacement of optimized topology magnified 10 times; (c) Convergence of objective function and discreteness during optimization. 


\subsubsection{Interior/Exterior Surfaces}

The Interior/Exterior Surfaces result is presented in Figure 4.4a. Again, the actuator's walls are enlarged at specific regions to promote the motion in desired direction $\left(J(\boldsymbol{u})=5.7 \mathrm{~mm}^{2}\right)$. Also, the discreteness level is low $\left(M_{n d}=0.7 \%\right)$ indicating an almost black and white solution.

The deformed configuration of Figure 4.4b presents a red contour to indicate the undeformed configuration. The right bottom tip of the actuator moves downward in the desired direction $\boldsymbol{l}_{\text {out }}=[0,-1]^{T}$ showing that the optimization objective is achieved. The displacement in Figure $4.4 \mathrm{~b}$ is magnified 10 times to help visualization.

The convergence curves of objective function $(J(\boldsymbol{u}))$ and discreteness $\left(M_{n d}\right)$ are presented in Figure 4.4c. As in the Interior Surface plot (Figure 4.3c), the objective function curve is plotted as a solid blue line and the discreteness curve is plotted as a dashed red line. The convergence graphs present less steps due to continuation of $\beta$ parameter than in the Interior Surface example.

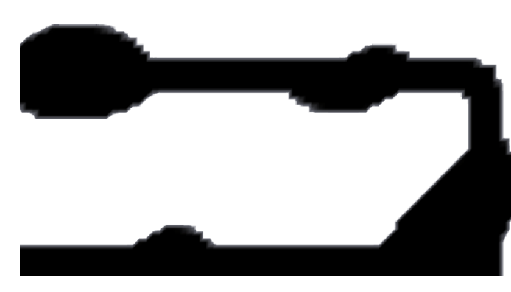

(a)

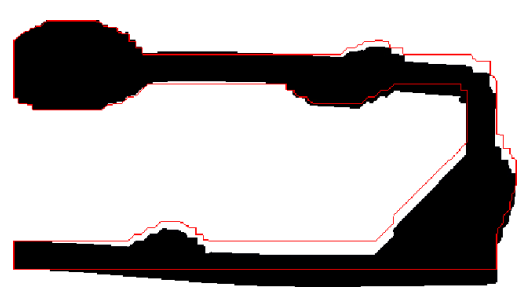

(b)

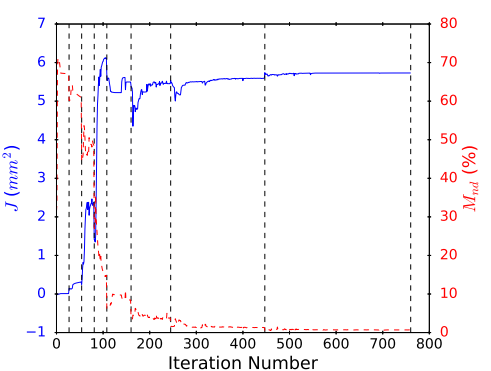

(c)

Figure 4.4 - Result of interior and exterior surfaces optimization for bending actuator problem. (a) Optimized topology; (b) Displacement of optimized topology magnified 10 times; (c) Convergence of objective function and discreteness during optimization.

\subsubsection{Barrier Projection}

The bending actuator optimization using Barrier Projection produces the result presented in Figure 4.5a. The objective function value for this design is $J(\boldsymbol{u})=6.7 \mathrm{~mm}^{2}$ and the discreteness level is $M_{n d}=1.2 \%$. Once again, the discreteness level is low enough to consider that the design is composed of black and white pixels.

The deformed configuration of the design obtained with Barrier Projection is presented in Figure 4.5b. The red contour indicates the undeformed configuration. The displacement is magnified 10 times in Figure 4.5c for better visualization. 


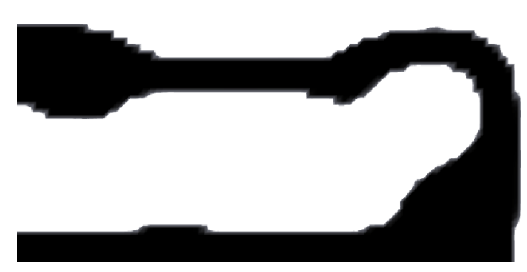

(a)

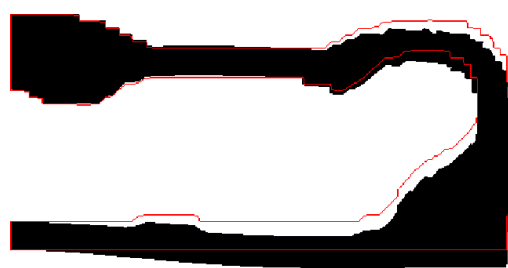

(b)

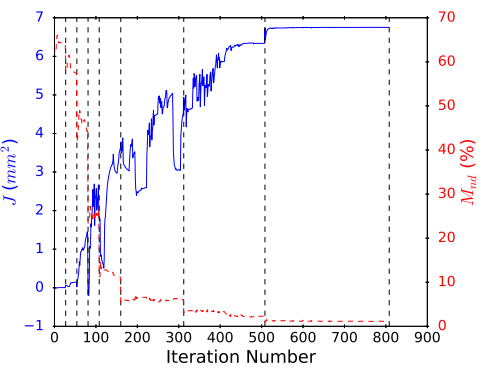

(c)

Figure 4.5 - Result of barrier optimization for bending actuator problem. (a) Optimized topology; (b) Displacement of optimized topology magnified 10 times; (c) Convergence of objective function and discreteness during optimization.

Table 4.3 - Number of finite elements and design variables for bending actuator optimization.

\begin{tabular}{lcc}
\hline Example & Elements & Design Variables \\
\hline Interior & 4608 & 1564 \\
Interior/Exterior & 6400 & 2476 \\
Barrier Projection & 6400 & 2117 \\
\hline
\end{tabular}

\subsubsection{Comparison of Results}

The number of finite elements required for bending actuator optimization is different when optimizing just the Interior Surfaces than when optimizing Interior/Exterior Surfaces and when applying Barrier Projection for optimization because the later approaches require the extension of the domain. Table 4.3 presents the number of elements and design variables used for each approach.

The calculation to find the number of finite element is presented in Equation 4.2.

$$
N_{F E M}=2 \frac{L_{D} H_{D}}{e l_{\text {size }} e l_{\text {size }}}
$$

The number of design variables is different in all approaches because there are differences in design domain size as discussed above for the number of finite elements, but also because there are different regions of fixed design variables in domain. Table 4.3 summarizes the number of design variables used in each approach.

The results obtained for the bending actuator (Figures 4.3a, 4.4a, 4.5a) present similar features: the upper fixed part is enlarged and the corner close to output port is also enlarged. These similar features show that the results are in accordance.

Table 4.4 presents a summary of the results for bending actuator optimization. The discreteness level for all approaches is sufficient to consider that the solutions are $0-1$ solutions. 
Table 4.4 - Bending actuator example summary.

\begin{tabular}{lcc}
\hline Example & $J(\boldsymbol{u})\left(\mathrm{mm}^{2}\right)$ & $M_{n d}(\%)$ \\
\hline Interior & 4.9 & 2.5 \\
Interior/Exterior & 5.7 & 0.7 \\
Barrier Projection & 6.7 & 1.2 \\
\hline
\end{tabular}

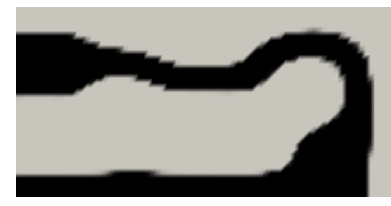

(a) $k_{\text {out }}=10^{-7}$

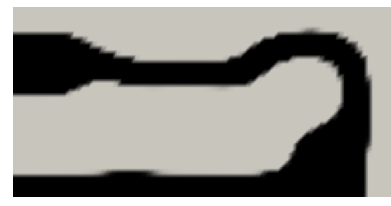

(b) $k_{\text {out }}=10^{-5}$

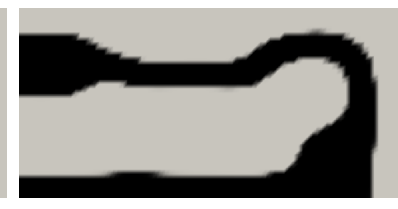

(c) $k_{\text {out }}=10^{-3}$

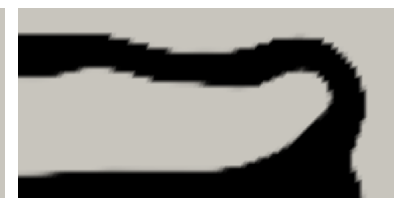

(d) $k_{\text {out }}=10^{-1}$

Figure 4.6 - Bending actuator results for different values of output port stiffness $\left(k_{\text {out }}\right)$ in $\mathrm{N} / \mathrm{mm}$ (see Table 4.5).

\subsubsection{Effect of Output Port Stiffness}

The output port stiffness $k_{\text {out }}$ models the stiffness of the workpiece. According to literature (PEDERSEN; BUHL; SIGMUND, 2001), lower values of $k_{\text {out }}$ produces more compliant actuators which have higher magnitude of output port displacement while higher values of $k_{\text {out }}$ produces stiffer actuators which have higher magnitude of output port force. So, lower values of $k_{\text {out }}$ is used for emphasis on displacement generation and higher values of $k_{\text {out }}$ is used for emphasis on force generation. The force on output port can be evaluated by a mean force $\bar{F}_{\text {out }}$ given by the product of $k_{\text {out }}$ by the objective function $J(\boldsymbol{u})$ divided by the output port size $\left\|\Gamma_{\text {out }}\right\|$ (Equation 4.3 ) because the force on a spring is the product of the spring stiffness by its displacement (Equation 2.69).

$$
\bar{F}_{\text {out }}=\frac{k_{\text {out }}}{\left\|\Gamma_{\text {out }}\right\|} J(\boldsymbol{u})=\frac{k_{\text {out }}}{\left\|\Gamma_{\text {out }}\right\|} \int_{\Gamma_{\text {out }}} \boldsymbol{l}_{\text {out }} \cdot \boldsymbol{u} d \Gamma
$$

To check if results using barrier projection are in accordance with this expected behavior, the optimization is run for different values of $k_{\text {out }}$ and the results are presented in Figure 4.6 and Table 4.5. According to Table 4.5, results with higher values of $k_{\text {out }}$ have lower values of objective function $J(\boldsymbol{u})$ which is related to output port displacement $\boldsymbol{u}$. So, the results are in accordance with literature: higher values of $k_{\text {out }}$ produces force generation mechanisms and lower values of $k_{\text {out }}$ produces displacement generation mechanisms.

\subsubsection{Initial Guess Effect}

The effect of initial guess is evaluated by starting the optimization from the design fields of Figures 4.7a and 4.8a. The density filter parameters for these examples are $r=3 \mathrm{~mm}$ and $l$ $=3 \mathrm{~mm}$. Small values are chosen to avoid that the density filter closes the holes from initial 
Table 4.5 - Bending actuator results for different values of output port stiffness $\left(k_{\text {out }}\right)$ in $\mathrm{N} / \mathrm{mm}$ (see Figure 4.6).

\begin{tabular}{lcccc}
\hline Topology & $k_{\text {out }}(\mathrm{N} / \mathrm{mm})$ & $J(\boldsymbol{u})\left(\mathrm{mm}^{2}\right)$ & $\bar{F}_{\text {out }}(N)$ & $M_{n d}(\%)$ \\
\hline Figure 4.6a & $10^{-7}$ & $9.3 \times 10^{0}$ & $9.3 \times 10^{-8}$ & 1.3 \\
Figure 4.6b & $10^{-5}$ & $9.1 \times 10^{0}$ & $9.1 \times 10^{-6}$ & 1.4 \\
Figure 4.6c & $10^{-3}$ & $7.7 \times 10^{0}$ & $7.7 \times 10^{-4}$ & 1.1 \\
Figure 4.6d & $10^{-1}$ & $3.0 \times 10^{-1}$ & $3.0 \times 10^{-3}$ & 1.6 \\
\hline
\end{tabular}

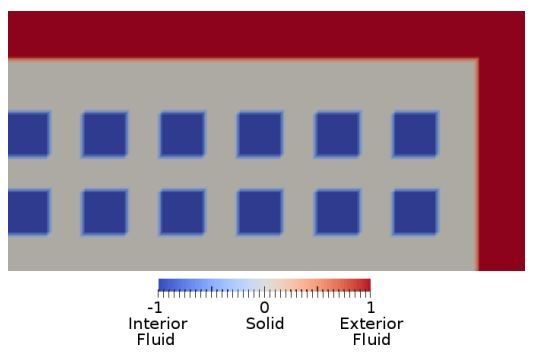

(a)

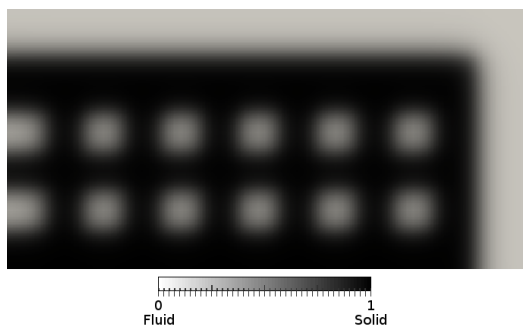

(b)

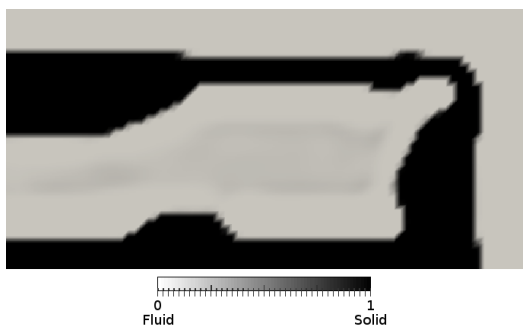

(c)

Figure 4.7 - Bending actuator optimization using an initial guess with square holes of $15 \mathrm{~mm}$. (a) Initial guess on design variable field; (b) Initial guess projected on physical density field; (c) Result on physical density field.

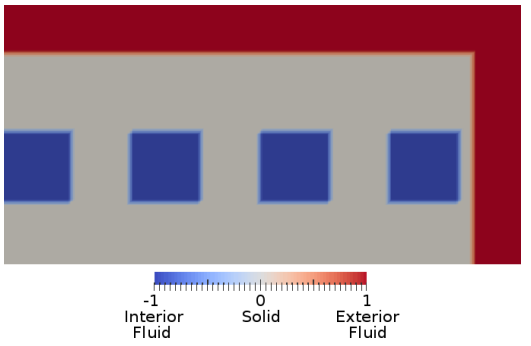

(a)

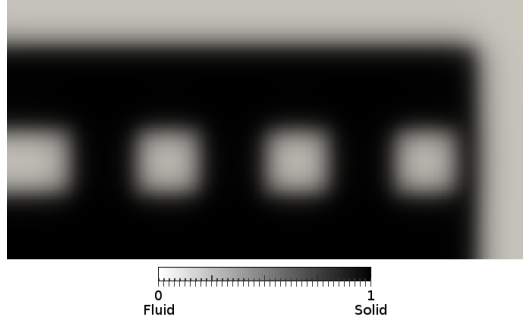

(b)

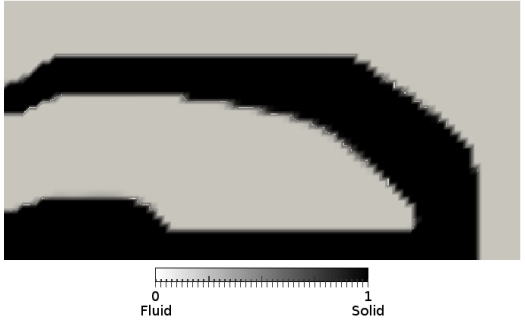

(c)

Figure 4.8 - Bending actuator optimization using an initial guess with square holes of $25 \mathrm{~mm}$. (a) Initial guess defined on design variable field; (b) Initial guess projected on physical density field; (c) Result on physical density field.

guess. In fact, the filters do not close the initial guess holes as can be seen from the physical density fields from Figures $4.7 \mathrm{~b}$ and $4.8 \mathrm{~b}$.

The results from Figures 4.7c and 4.8c show that the initial guess influences the optimization result as expected, because the design of complaint mechanisms by topology optimization is a non-convex optimization problem. Also, these examples show the ability of the barrier projection technique in changing topological features, because the holes presented in initial guesses are removed during optimization because they are not optimal. 


\subsubsection{Effect of Filter Parameters $r$ and I}

The effect of filter parameters $r$ and $l$ on barrier projection optimization result is evaluated by running the optimization for different values of $r$ and $l$. Five lengths are used during the evaluation: $3,4,5.5,7$, and $8 \mathrm{~mm}$ and the results for each combination of $r$ and $l$ are presented in Table 4.6. Each row has results for the same value of $r$ while each column has results for the same value of $l$.

According to Table 4.6, for a fixed value of $l$, the minimum length size decreases as $r$ increases. This can be observed by comparing the results of a single column. On the other hand, for a fixed value of $r$, the minimum length size increases as $l$ increases and this can be checked by comparing the results of a single row.

Some optimizations ended with "converged to point of local infeasibility" exit code. In this case, the IPOPT documentation suggests running the simulation again for a different initial guess to check if the problem is really infeasible. Following this suggestion, the optimizations that failed are executed starting from the initial guess of Figure 4.7. The results marked with a blue dot successfully converged after changing the initial guess. However, the test cases where the results is marked with a green dot still converged to a point of local infeasibility with the new initial guess. The results marked with a green dot are obtained by setting the tolerance to $\epsilon_{t o l}=10^{-3}$ instead of changing the initial guess. Finally, the result marked with a red dot is obtained by setting $\epsilon_{t o l}=10^{-3}$ and the initial guess from Figure 4.8, because other attempts failed with convergence to points of local infeasibility.

\subsubsection{Finite Deformation}

The result of optimization by using Barrier Projection (Figure 4.5a) is used in simulations considering geometrical and material nonlinearities (Appendix B) to check if the behavior under large displacements is also in accordance with design objective. The results are presented in Figure 4.9 and Table 4.7. The mesh used for nonlinear analysis is created from the result of Figure 4.5 a by taking only the triangles with physical density greater than 0.8 .

Figure 4.9 presents the deformation for $p_{i n}=7.6 \mathrm{kPa}$ which is around the maximum input pressure value for which the problem converges. It is possible to see that output port moves in the downward direction as desired. There is no magnifying of displacement in Figure 4.9.

Table 4.7 presents the output displacement for different levels of input pressure. The third column of Table 4.7 shows that the relation between output displacement and input pressure is nonlinear. It also shows the strain stiffening effect, because the displacement to pressure ratio decreases as the input pressure increases.

By simulating the nonlinear model with an input pressure of $p_{\text {in }}=0.1 \mathrm{kPa}$, the output displacement obtained is $J(\boldsymbol{u})=4.1 \mathrm{~mm}^{2}$ according to Table 4.8. This result is different from 
Table 4.6 - Summary of density filter parameters $r$ and $l$ effect on barrier projection optimization result. Each row presents results for the same value of $r$ and each column presents results for the same value of $l$. The results marked with a blue dot use the initial guess from Figure 4.7. The result marked with a green dot uses $\epsilon_{t o l}=10^{-3}$. The result marked with a red dot uses $\epsilon_{t o l}=10^{-3}$ and the initial guess of Figure 4.8. The use of different tolerance and initial guesses is made to avoid converge to points of local infeasibility.

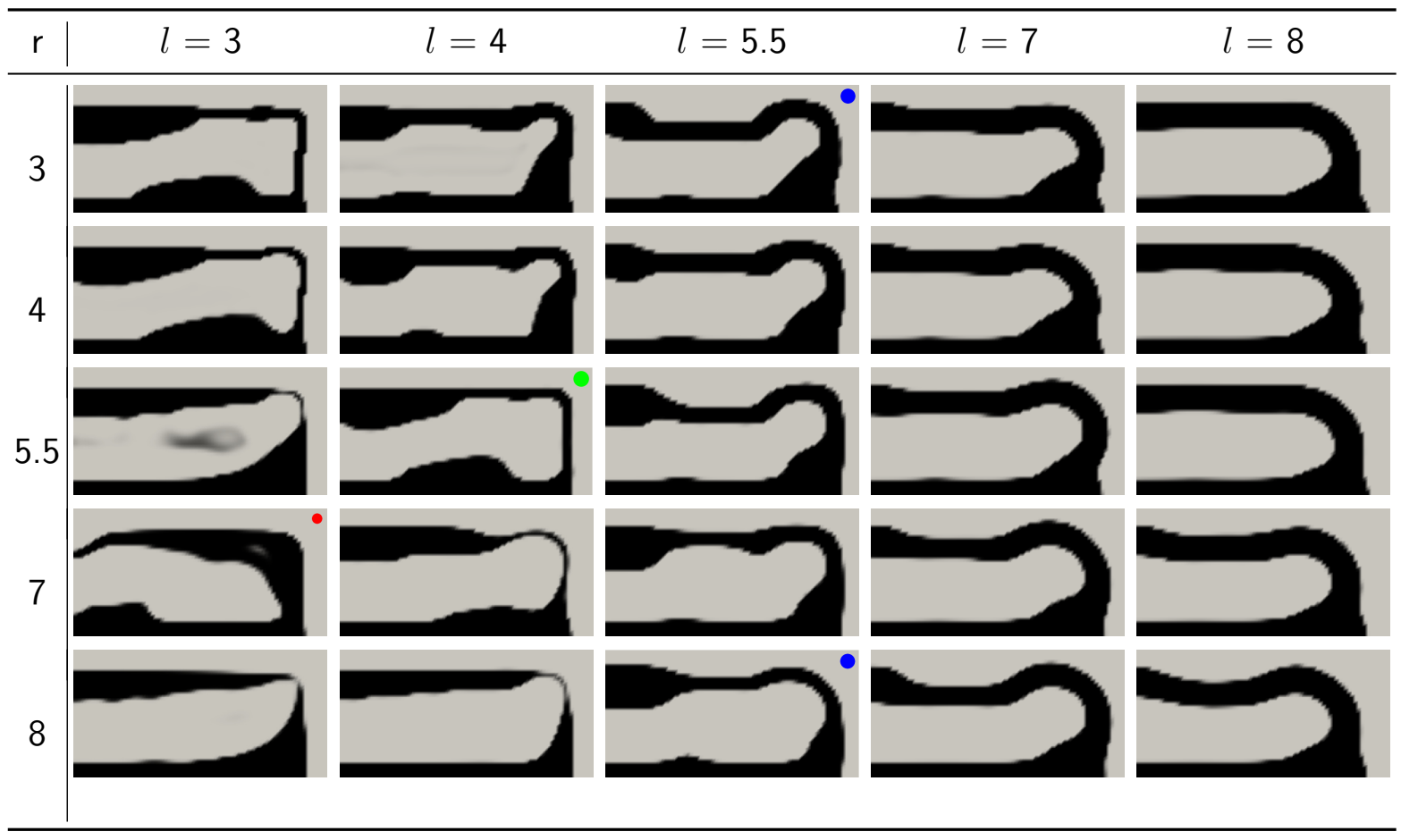

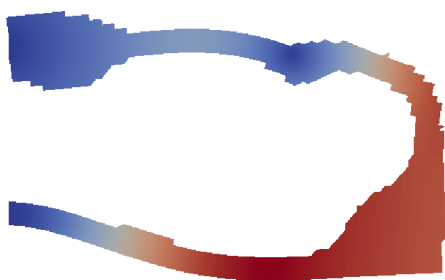

Figure 4.9 - Finite deformation of bending actuator for $p_{i n}$ $=7.6 \mathrm{kPa}$.

\begin{tabular}{ccc}
\hline$p_{\text {in }}(\mathrm{kPa})$ & $J(\boldsymbol{u})\left(\mathrm{mm}^{2}\right)$ & $J / p_{\text {in }}$ \\
\hline 7.6 & 208.0 & $-2.7 \mathrm{E}+4$ \\
5.4 & 167.2 & $-3.1 \mathrm{E}+4$ \\
3.2 & 111.9 & $-3.5 \mathrm{E}+4$ \\
1.0 & 39.4 & $-3.9 \mathrm{E}+4$ \\
\hline
\end{tabular}

Table 4.7-Finite deformation of bending actuator for different values of input pressure $p_{i n}$. 
Table 4.8 - Output displacement of bending actuator by considering different FEM models for a input pressure $p_{i n}=0.1 \mathrm{kPa}$. The second, third, and forth rows use the same mesh used for Figure 4.9.

\begin{tabular}{lc}
\hline Model & $J(\boldsymbol{u})\left(\mathrm{mm}^{2}\right)$ \\
\hline Optimization Result & 6.7 \\
Mixed $\boldsymbol{u}$-p (plane stress) & 5.5 \\
Standard Displacement (plane stress) & 4.7 \\
Nonlinear & 4.1 \\
\hline
\end{tabular}

the output displacement obtained during optimization which is $6.7 \mathrm{~mm}^{2}$, although the material constants are selected for compatibility between the linear and nonlinear models under small deformations. The difference is caused by the different mesh and different function spaces used for function approximation. Table 4.8 also presents results for simulations using mixed $\boldsymbol{u}$-p and standard displacement formulations.

\subsection{Linear Actuator}

The Linear Actuator problem aims to find the optimized actuator in which the output port displacement is in the same direction of the resulting force over the inlet (input port) surface. Once again, the design domain for the different approaches followed in this work are different. The design domains are presented in Figure 4.10 where regions in white are free during optimization (not fixed), regions in black are fixed to be solid, and regions in gray are fixed to be exterior fluid.

The output port direction is $\boldsymbol{l}_{\text {out }}=[1,0]^{T}$, because the input pressure $p_{\text {in }}$ acts on the inlet at left edge producing a resulting force in the horizontal direction pointing rightward.

The design domain has dimensions $L_{D}=L$ and $H_{D}=H$ for the Interior Surface optimization problem and $L_{D}=L$ and $H_{D}=H+2 H_{E}$ for the Interior/Exterior Surface and Barrier Projection optimizations. Again, the reason for extending the domain is to enable the optimization of exterior surface in Interior/Exterior problem and to ensure the existence of exterior fluid and, consequently, solid material between interior fluid and exterior fluid in Barrier Projection problem.

For the Interior and Interior/Exterior problems, an enclosure of thickness $t$ is fixed as solid at design variable field to guarantee that the actuator remains closed during optimization (Figures 4.10a and 4.10b).

In Barrier Projection problem, the fixed solid region at output port has height $H-2\left(t+L_{I}\right)$. The reason for reducing the height of output port by $t+L_{I}$ at both sides is to obtain an actuator with a similar size of Interior and Interior/Exterior problems. In this way, the comparison 


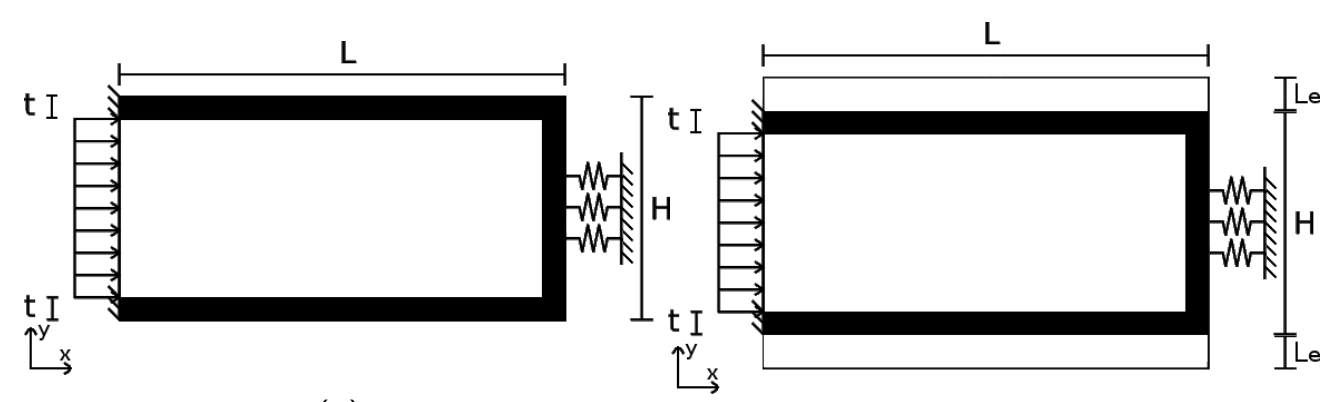

(a)

(b)

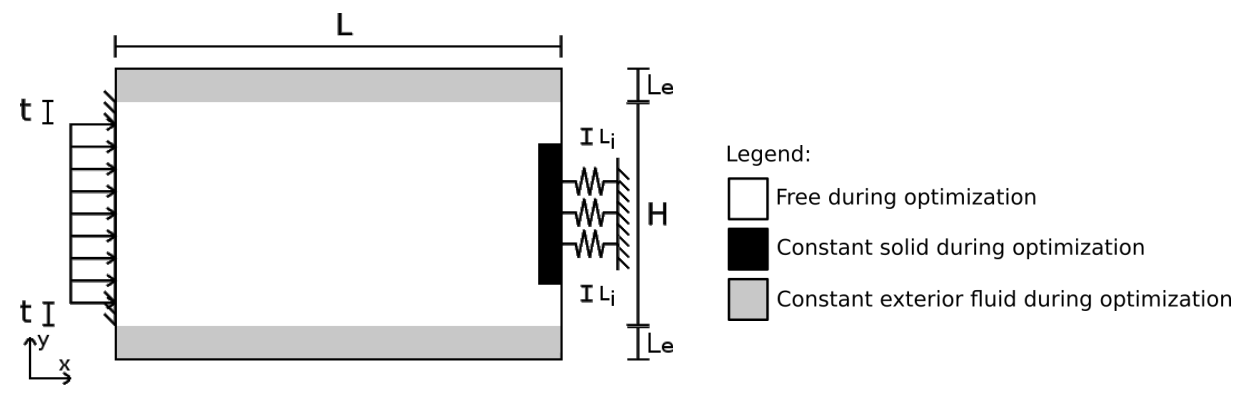

(c)

Figure 4.10 - Design domain for linear and inverter actuators problems. (a) Interior Surface; (b) Interior/Exterior Surfaces; (c) Barrier Projection.

between the results is fairer.

The selected dimensions for linear actuator problem are $L=80 \mathrm{~mm}, H=80 \mathrm{~mm}, L_{E}$ $=20 \mathrm{~mm}, L_{I}=10 \mathrm{~mm}$, and $t=10 \mathrm{~mm}$. The aspect ratio is reduced in linear actuator in relation to bending actuator to reduce optimization time, since crossed finite elements are used instead of right triangular finite elements in favor of symmetry. The allowed material volume is $V^{*}=V_{\text {frac }} L H$ with $V_{\text {frac }}=50 \%$ for all approaches (considering unit depth).

The termination criteria for linear actuator problem is presented in Equation 4.4.

$$
\begin{aligned}
& \epsilon_{\text {tol }}=10^{-5} \\
& n_{\text {max }}=\left[\begin{array}{llllllll}
25 & 25 & 25 & 25 & 50 & 150 & 200 & 500
\end{array}\right]^{T}
\end{aligned}
$$

\subsubsection{Interior Surface}

The result for Interior Surface optimization of linear actuator is presented in Figure 4.11a. The upper and lower walls of the actuator are enlarged to reduce deformation, because the deformation of upper and lower walls reduce the length of upper and lower walls in horizontal direction, pulling the right wall in the opposite direction of the desired output port displacement.

Figure 4.11b presents the displacement of Figure 4.11a. The output port displacement is obtained just by the deformation of right wall. Consequently it is small even when magnified 250 times. 
The convergence graphs for objective function (blue) and discreteness (red) are presented in Figure 4.11c. The end of each continuation iteration is marked by a dashed vertical line. It is possible to see that the continuation of parameters produce steps in convergence graphs.

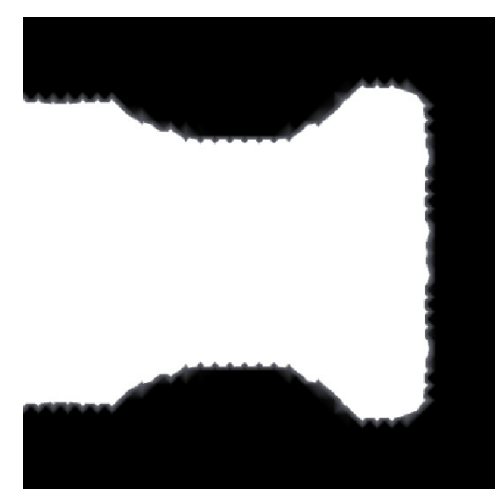

(a)

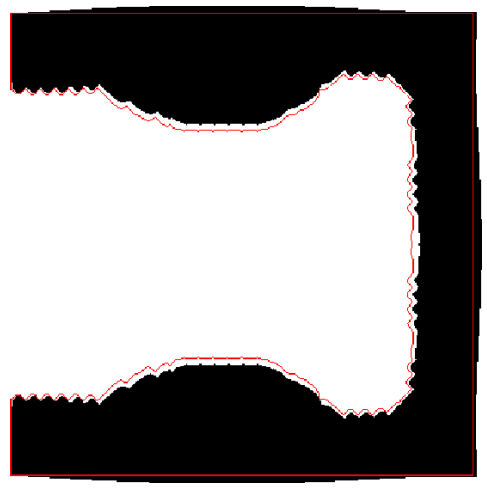

(b)

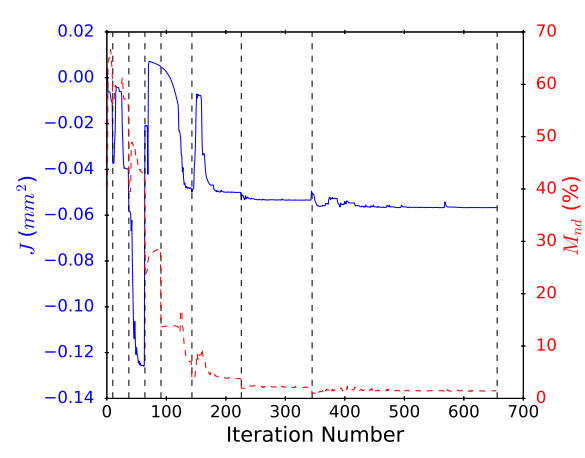

(c)

Figure 4.11 - Result of interior surfaces optimization for linear actuator problem. (a) Optimized topology; (b) Displacement of optimized topology magnified 250 times; (c) Convergence of objective function and discreteness during optimization.

\subsubsection{Interior/Exterior Surfaces}

The result for Interior/Exterior Surfaces optimization is shown in Figure 4.12a and it presents similar features as the result of Interior Surface optimization (Figure 4.11a): the upper and lower walls are enlarged to reduce deformation in vertical direction, because the deformation in vertical direction reduces the horizontal length of upper and lower walls, pulling the right wall in a direction opposed to desired output port direction $\boldsymbol{l}_{\text {out }}=[1,0]^{T}$.

Figure 4.12c presents the convergence graphs. Again, it is possible to verify steps in convergence associated to continuation of $\beta$ parameter (marked by vertical dashed lines).

\subsubsection{Barrier Projection}

The shape of Barrier Projection result presented in Figure 4.13a is different from the results obtained in Interior and Interior/Exterior approaches (Figures 4.11a and 4.12a). Upper and lower walls are curved to the interior of the actuator. When pressure is applied, upper and lower walls deforms outward increasing the length of these wall in horizontal direction. The right wall is pulled rightward increasing output port displacement in desired direction $\boldsymbol{l}_{\text {out }}=[1,0]^{T}$.

\subsubsection{Comparison of Results}

As in the example of bending actuator, the number of finite elements and design variables are different in all approaches because there are differences in design domain size and differences 


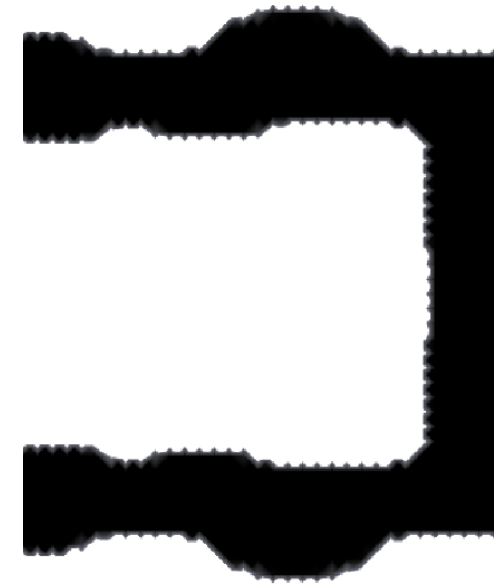

(a)

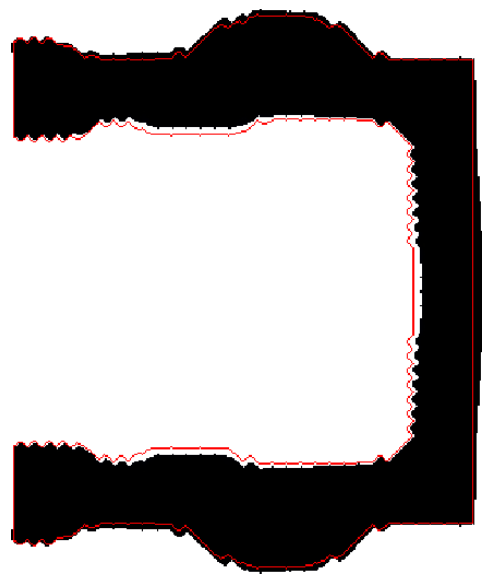

(b)

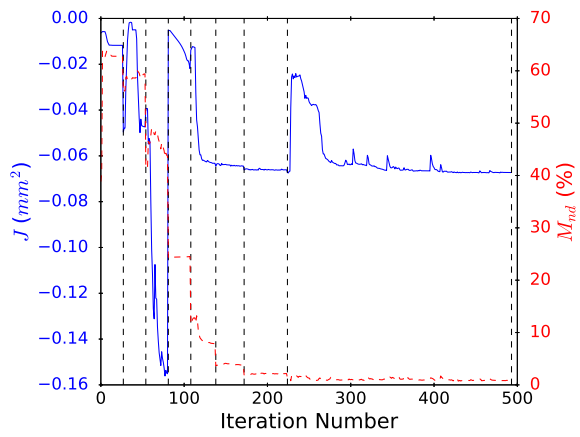

(c)

Figure 4.12 - Result of interior and exterior surfaces optimization for linear actuator problem. (a) Optimized topology; (b) Displacement of optimized topology magnified 250 times; (c) Convergence of objective function and discreteness during optimization.

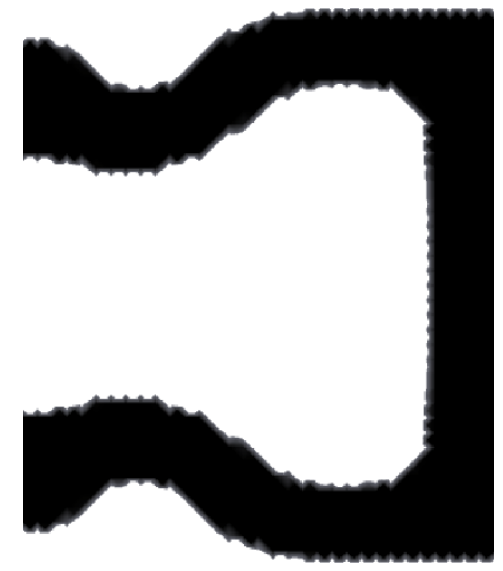

(a)

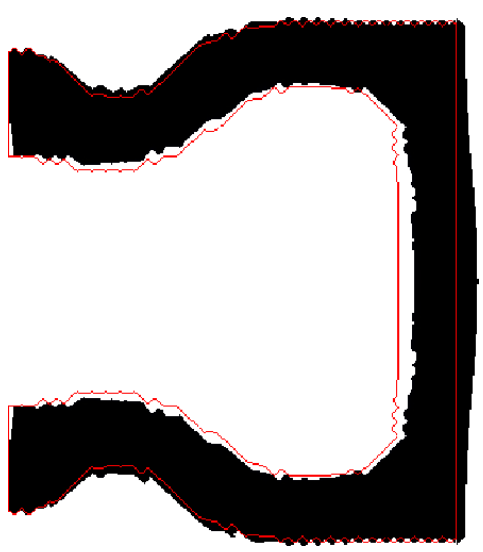

(b)

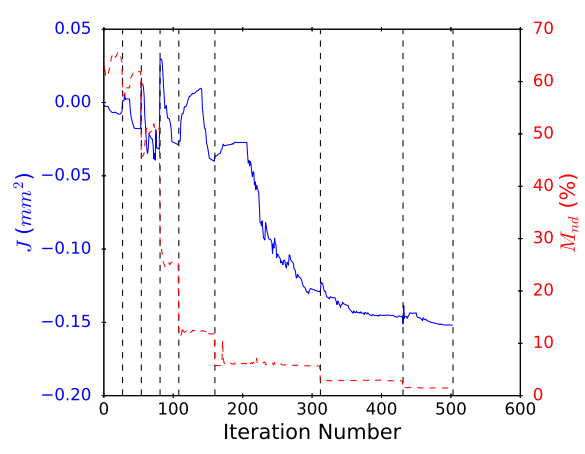

(c)

Figure 4.13 - Result of barrier optimization for linear actuator problem. (a) Optimized topology; (b) Displacement of optimized topology magnified 250 times; (c) Convergence of objective function and discreteness during optimization.

in fixed design variables regions. Table 4.9 summarizes the number of finite elements and design variables used in each approach.

Table 4.10 summarizes the results of Linear Actuator optimization. The objective function column shows that the Barrier Projection result presents a displacement twice larger than Interior and Interior/Exterior results. As actuators from Figures 4.11a, 4.12a, and 4.13a present right walls of similar size, the difference in output displacement is due to upper and lower walls. 
Table 4.9 - Number of finite elements and design variables for linear and inverter actuator optimization.

\begin{tabular}{lcc}
\hline Example & Elements & Design Variables \\
\hline Interior & 4096 & 1316 \\
Interior/Exterior & 6400 & 2356 \\
Barrier Projection & 6400 & 1941 \\
\hline
\end{tabular}

Table 4.10 - Linear actuator example summary.

\begin{tabular}{lcc}
\hline Example & $J(\boldsymbol{u})\left(\mathrm{mm}^{2}\right)$ & $M_{n d}(\%)$ \\
\hline Interior & 0.06 & 1.4 \\
Interior/Exterior & 0.07 & 0.9 \\
Barrier Projection & 0.15 & 1.4 \\
\hline
\end{tabular}

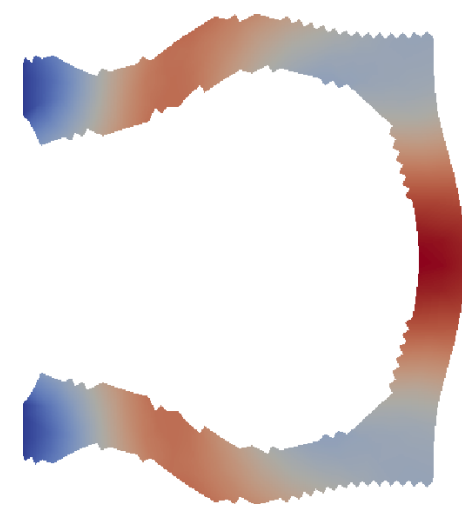

Figure 4.14 - Finite deformation of linear actuator for $p_{\text {in }}$ $=0.18 \mathrm{MPa}$.

\begin{tabular}{ccc}
\hline$p_{\text {in }}(\mathrm{MPa})$ & $J(\boldsymbol{u})\left(\mathrm{mm}^{2}\right)$ & $J / p_{\text {in }}$ \\
\hline 0.18 & 125.6 & $7.0 \mathrm{E}+2$ \\
0.12 & 96.3 & $8.0 \mathrm{E}+2$ \\
0.07 & 64.6 & $9.2 \mathrm{E}+2$ \\
0.01 & 11.4 & $1.1 \mathrm{E}+3$
\end{tabular}

Table 4.11 - Finite deformation of linear actuator for different values of input pressure $p_{i n}$.

\subsubsection{Finite Deformation}

The result of Figure 4.13a is also simulated for large displacements (Appendix B) and the results are presented in Figure 4.14 and in Table 4.11. The mesh used for nonlinear analysis is created from Figure 4.13 a by taking only the triangles with physical density above 0.8 .

Figure 4.14 shows the deformation of the optimized linear actuator for an input pressure of $p_{i n}=0.18 \mathrm{MPa}$, which is the maximum value presented in Table 4.11 and it is around the maximum input pressure for which the problem converges.

As in the bending actuator problem, Table 4.11 shows that the relation (third column) between output displacement (second column) and input pressure (first column) is nonlinear and it decreases as the input pressure increases (strain stiffening).

It is also possible to compare the results of the nonlinear model with the linear models. The simulation of the nonlinear model for the same input pressure used during optimization 
Table 4.12 - Output displacement of linear actuator by considering different FEM models for a input pressure $p_{i n}=1 \mathrm{kPa}$. The second, third, and forth rows use the same mesh used for Figure 4.14.

\begin{tabular}{lc}
\hline Model & $J(\boldsymbol{u})\left(\mathrm{mm}^{2}\right)$ \\
\hline Optimization Result & 0.15 \\
Mixed $\boldsymbol{u}$-p (plane stress) & 0.14 \\
Standard Displacement (plane stress) & 0.12 \\
Nonlinear & 0.12 \\
\hline
\end{tabular}

$\left(p_{\text {in }}=0.1 \mathrm{kPa}\right.$ ) produces an output port displacement of $J(\boldsymbol{u})=0.12 \mathrm{~mm}^{2}$ that is different from $0.15 \mathrm{~mm}^{2}$ obtained during optimization. This difference is associated to the different mesh and finite element function space used. If a linear model is used with the same mesh the output port displacement is also $J(\boldsymbol{u})=0.12 \mathrm{~mm}^{2}$.

\subsection{Inverter Actuator}

The Inverter Actuator is designed on the same design domain of the Linear Actuator (Figure 4.10) and it also uses the same parameters. However, the Inverter Actuator problem seeks the optimized material distribution whose output port displacement is in the opposed direction of the resultant force on input port due to pressure loading. As the input port is the left edge, the output port direction is $\boldsymbol{l}_{\text {out }}=[-1,0]^{T}$.

\subsubsection{Interior Surface}

The result for the topology optimization of the Interior Surface of the Inverter Actuator is presented in Figure 4.15. According to Figure 4.15b, the material distribution of Figure 4.15a presents output displacement in the desired direction $\boldsymbol{l}_{\text {out }}=[-1,0]^{T}$.

The displacement of output port is obtained by leaving the upper and lower edges thinner and enlarging upper right and lower right corners. The enlargement of these corners reduce its deformation and, consequently, they only rotate. The rotation of upper corner in counter clockwise direction is due to upper edge movement upwards while the rotation of lower corner in clockwise direction is due to lower edge movement downwards. The combination of these rotations makes the right wall to bend inward.

\subsubsection{Interior/Exterior Surfaces}

The Interior/Exterior optimization of Inverter Actuator produces the result of Figure 4.16a. The displacement of this result is in accordance with the objective function requirement according to $4.16 \mathrm{~b}$. 


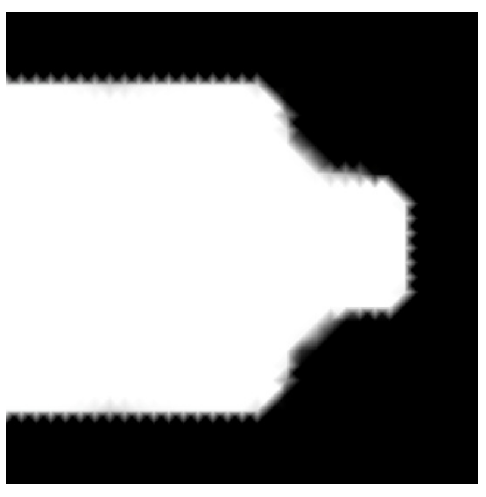

(a)

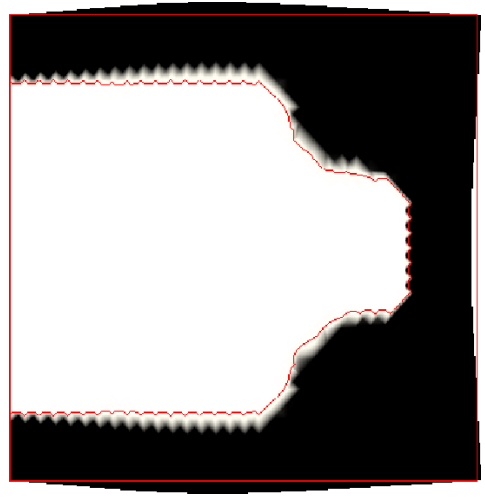

(b)

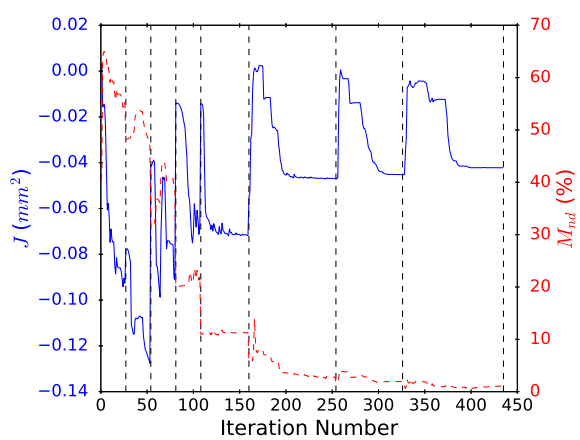

(c)

Figure 4.15 - Result of interior surfaces optimization for inverter actuator problem. (a) Optimized topology; (b) Displacement of optimized topology magnified 250 times; (c) Convergence of objective function and discreteness during optimization.

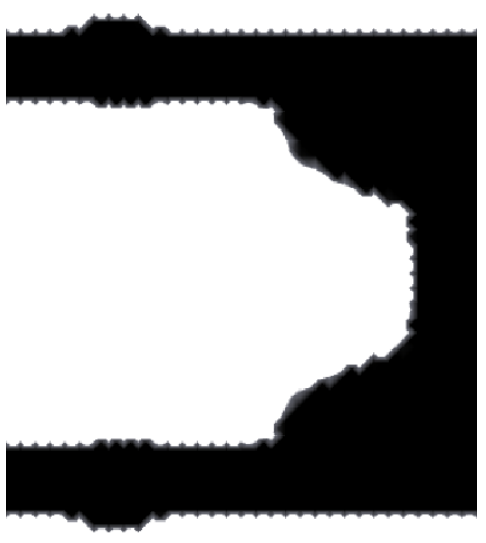

(a)

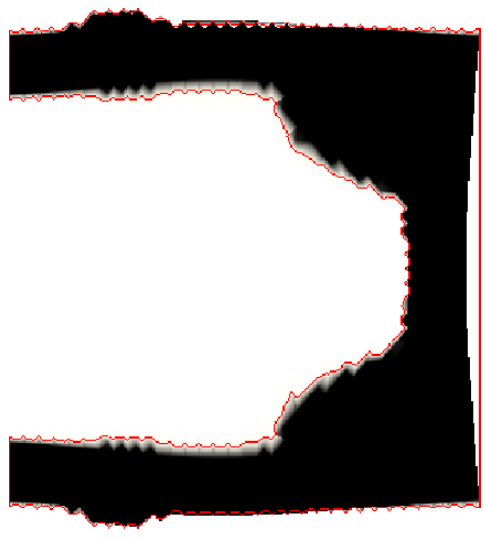

(b)

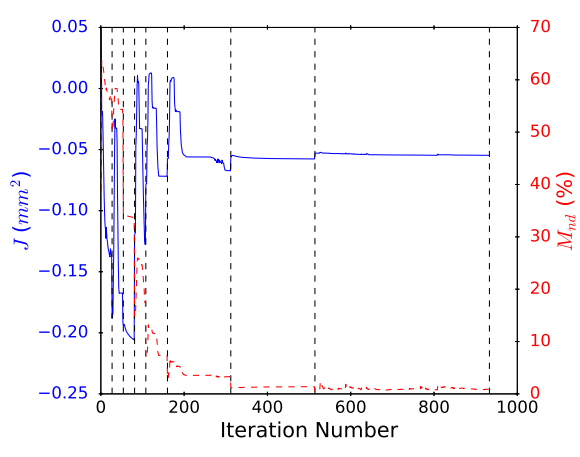

(c)

Figure 4.16 - Result of interior and exterior surfaces optimization for inverter actuator problem. (a) Optimized topology; (b) Displacement of optimized topology magnified 250 times; (c) Convergence of objective function and discreteness during optimization.

The topology of Figure 4.16a is similar to the topology of Figure 4.15a. Consequently, the movement mechanism is the same as described in Interior Surfaces section.

\subsubsection{Barrier Projection}

The result of Barrier Projection optimization for Inverter Actuator uses the same approach as Interior and Interior/Exterior results for moving in output port direction. However, the curved and thinner upper and lower edges deform more than in Interior and Interior/Exterior and the output displacement is larger. 


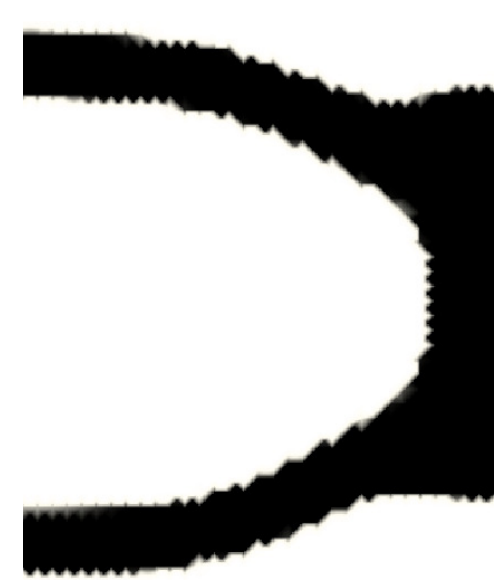

(a)

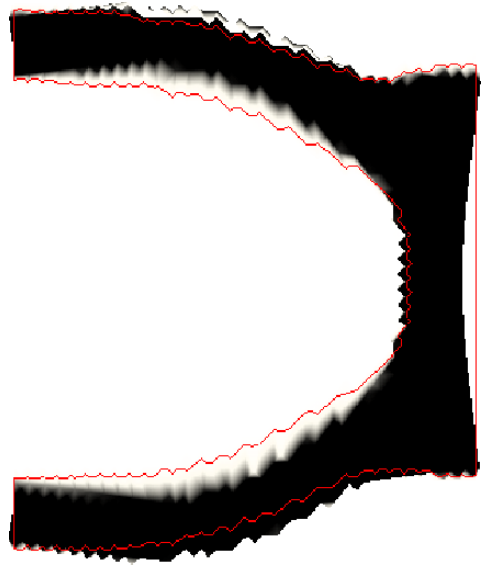

(b)

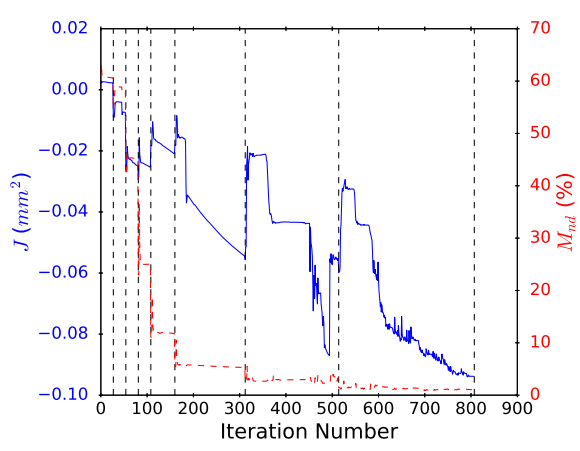

(c)

Figure 4.17 - Result of barrier optimization for inverter actuator problem. (a) Optimized topology; (b) Displacement of optimized topology magnified 250 times; (c) Convergence of objective function and discreteness during optimization.

Table 4.13 - Inverter actuator example summary.

\begin{tabular}{lcc}
\hline Example & $J(\boldsymbol{u})\left(\mathrm{mm}^{2}\right)$ & $M_{n d}(\%)$ \\
\hline Interior & 0.04 & 1.1 \\
Interior/Exterior & 0.05 & 0.9 \\
Barrier Projection & 0.09 & 1.1 \\
\hline
\end{tabular}

\subsubsection{Comparison of Results}

The number of finite elements and design variables for Inverter problem is equal to the numbers for Linear problem. They are presented in Table 4.9.

The absolute value of objective function $J(\boldsymbol{u})$ indicates that the topology obtained with Barrier Projection for the Inverter Actuator problem generates greater displacement at output port than the topologies obtained with Interior and Interior/Exterior approaches according to Table 4.13.

\subsubsection{Finite Deformation}

The nonlinear analysis (Appendix B) of the result of Inverter Actuator optimization by Barrier Projection (Figure 4.17a) is presented in Table 4.14. The analysis is carried out for different load levels. Again, the mesh used in nonlinear analysis is extracted from optimization result (Figure $4.17 \mathrm{a}$ ) by considering only the triangles with physical density above 0.8 .

It is possible to see in Table 4.14 that the relation between pressure load and output displacement decreases as pressure load increases (according to third column). This is the strain stiffening effect once again. 


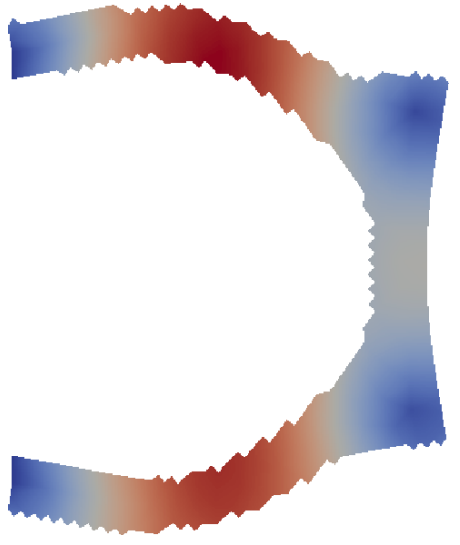

Figure 4.18 - Finite deformation of inverter actuator for $p_{\text {in }}$ $=0.095 \mathrm{MPa}$.

\begin{tabular}{ccc}
\hline$p_{\text {in }}(\mathrm{kPa})$ & $J(\boldsymbol{u})\left(\mathrm{mm}^{2}\right)$ & $J / p_{\text {in }}$ \\
\hline 95 & 30.8 & $3.2 \mathrm{E}+2$ \\
67 & 26.6 & $4.0 \mathrm{E}+2$ \\
38 & 18.6 & $4.9 \mathrm{E}+2$ \\
10 & 5.9 & $5.9 \mathrm{E}+2$ \\
\hline
\end{tabular}

Table 4.14 - Finite deformation of inverter actuator for different values of input pressure $p_{i n}$.

Table 4.15 - Output displacement of inverter actuator by considering different FEM models for a input pressure $p_{i n}=0.1 \mathrm{kPa}$. The second, third, and forth rows use the same mesh used for Figure 4.18.

\begin{tabular}{lc}
\hline Model & $J(\boldsymbol{u})\left(\mathrm{mm}^{2}\right)$ \\
\hline Optimization Result & 0.09 \\
Mixed $\boldsymbol{u}$-p (plane stress) & 0.08 \\
Standard Displacement (plane stress) & 0.06 \\
Nonlinear & 0.06 \\
\hline
\end{tabular}

The displacement field for $p_{i n}=0.095 \mathrm{MPa}$ is presented in Figure 4.18. It is possible to see that output port bends inwards in desired direction $\boldsymbol{l}_{\text {out }}=[-1,0]^{T}$ while the upper and lower wall deforms upward and downward, respectively. So, the optimized topology produces the desired movement under finite deformation.

The pressure load used during optimization is $p_{i n}=0.1 \mathrm{kPa}$ and the output displacement of nonlinear model for this pressure is $J(\boldsymbol{u})=-0.06 \mathrm{~mm}^{2}$. During optimization with the mixed model, the value obtained is $J(\boldsymbol{u})=-0.09 \mathrm{~mm}^{2}$. This difference is mainly caused by the mesh and function spaces used. This is verified by running a standard displacement linear analysis using the same mesh which produces $J(\boldsymbol{u})=-0.06 \mathrm{~mm}^{2}$.

\subsection{Hydraulic Actuators}

The previous sections considered the optimization of pneumatic actuators pressurized by air with $G_{F} \approx 0$ and $K_{F}=101 \mathrm{kPa}$ according to listing 2.71. Hydraulic actuators on the other hand are pressurized by liquids which have greater values of bulk modulus. So, this section presents optimization results when water with $K_{F}=2.15 \mathrm{GPa}$ is considered. Only the barrier projection approach is applied, because the results of the pneumatic actuators show that the 


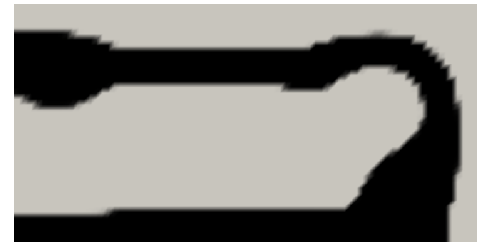

(a)

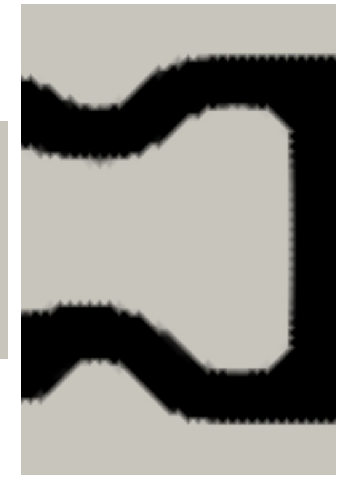

(b)

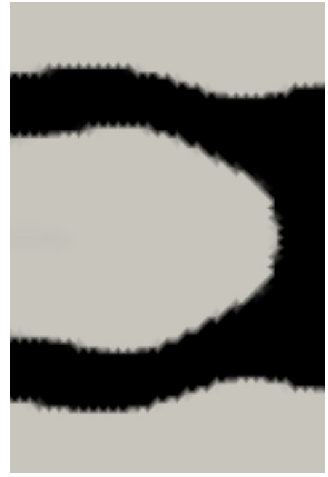

(c)

Figure 4.19 - Topology optimization results by considering water material properties in fluid phase. (a) Hydraulic bending actuator; (b) Hydraulic linear actuator; (c) Hydraulic inverter actuator.

Table 4.16 - Hydraulic actuators example summary.

\begin{tabular}{lcc}
\hline Example & $J(\boldsymbol{u})\left(\mathrm{mm}^{2}\right)$ & $M_{n d}(\%)$ \\
\hline Bending & 3.7 & 0.9 \\
Linear & 0.13 & 1.6 \\
Inverter & 0.03 & 1.3 \\
\hline
\end{tabular}

results with barrier projection are superior.

The three types of actuators studied are optimized for hydraulic actuation and the optimization results in physical density field are presented in Figure 4.19. The same parameters used to obtain Figures 4.5a, 4.13a, and 4.17a are used to obtain Figures 4.19a, 4.19b, and $4.19 c$, respectively.

The topologies of hydraulic actuators are the same of pneumatic actuators, because the shape of the walls are similar and the number of holes are the same. However, the hydraulic and pneumatic actuators are not the same. In fact, the hydraulic actuators are stiffer as can be seen from the thickness of their walls and from comparison of output displacement from Table 4.16 and Tables 4.4 (6.7 $\mathrm{mm}^{2}$ for bending), 4.10 (0.15 $\mathrm{mm}^{2}$ for linear), and 4.13 (0.09 $\mathrm{mm}^{2}$ for inverter).

Hydraulic actuators are stiffer than pneumatic actuators because fluids with higher bulk modulus present less displacement than fluids with lower bulk modulus. If the fluid region has lower displacement, the solid region must also have lower displacement for compatibility in the fluid-structure interface. The only way of achieving less displacement in solid region for the same input pressure is to have stiffer walls. 


\section{CONCLUSIONS}

The topology optimization formulation developed and implemented in this work by the combination of mixed displacement-pressure finite elements and compliant mechanism objective function is capable of designing pneumatic and hydraulic actuators in the linear material regime. This formulation requires the existence of solid material between fluids with different pressure, because the equations used are static and, therefore, they can not model pressure gradients in fluid. This requirement is verified analytically by examining that the stress tensor is hydrostatic when $G=0$ (Equation 2.33) and numerically by trying to optimize without fixing solid contours or applying barrier projection (Table 4.2).

The existence of solid material between fluids with different pressure is achieved in this work by fixing an enclosure of the actuator or by using the barrier projection technique proposed here (SOUZA; SILVA, 2020). It is possible to optimize the interior and exterior surfaces of the actuator while fixing an enclosure by extending the design domain. However, the approach of fixing an enclosure poses excessive constraints to the optimizer.

The barrier projection technique guarantees the existence of a solid material between exterior and interior fluid domains by setting different design variable values for each domain (i.e. solid, interior fluid, and exterior fluid) and by projecting domains with the same material properties to the same physical density value. In general, the barrier projection technique generates better results than fixing solid contours for the actuator, because the optimizer has more freedom to shape the actuator. The barrier projection technique is the main contribution of this work.

It is verified with numerical examples that barrier projection is capable of changing the topology of the design by starting with a perforated initial guess (Figures $4.7 \mathrm{~b}$ and $4.8 \mathrm{~b}$ ) and by checking that holes are removed during optimization (Figures 4.7c and 4.8c).

The design of three actuators is studied, namely bending, linear and inverter actuators. Both the approaches of fixing an enclosure and of using barrier projection are capable of solving the design problem for the three examples. For the bending and inverter examples, the barrier projection technique outperformed the fixing enclosure approach by producing more curved designs. For the linear actuator example, the barrier projection technique achieved superior performance by finding a better mechanism of obtaining motion in desired direction.

The three design problems are solved for pneumatic and hydraulic actuation by using the air bulk modulus for pneumatic actuation and by using the water bulk modulus for hydraulic actuation. The results obtained for pneumatic and hydraulic actuators have similar topology, i.e. number of holes and shape of actuator walls. The hydraulic actuators are stiffer, because 
the displacement of fluids with higher bulk modulus is smaller, enforcing the displacement of the solid region to also be smaller for compatibility in fluid-structure interface. Smaller displacements of solid region are only obtained for stiffer actuators.

The results of this work do not present hinges which are common features obtained when applying compliant mechanism formulations. As the input load is a pressure load and the movement is better achieved by the distribution of compliant regions over the whole domain instead of concentrating them, the designs are less inclined to present hinges.

\subsection{Future Work}

This work considers linear deformations and linear material model, although soft actuators are fabricated from elastomeric materials and present large deformation. Therefore, future work include the consideration of geometric and material nonlinearities.

Also, the results presented here are only for 2D models and next work include considering 3D models.

Here, only the actuator movement is considered during design, but another design objectives may also be considered in future works, such as the actuation time by minimizing the air volume for actuation. Also, more complex movements can be considered by selecting control points for trajectory matching.

The fabrication of the optimized topologies is also of interest because it can validate the obtained results experimentally.

New projection techniques that guarantee the existence of solid regions between exterior and interior are of interest to be compared with barrier projection technique.

Fluid-structure formulations for topology optimization are also of interest for modelling pressure gradient in fluid domain. Porous formulations could also be explored and the investigation of stress-constrained topology optimization formulations is also important. 


\section{REFERENCES}

ALLAIRE, G.; JOUVE, F.; TOADER, A.-M. A level-set method for shape optimization. Comptes Rendus Mathematique, Elsevier BV, v. 334, n. 12, p. 1125-1130, jan 2002. Available from Internet: <https://doi.org/10.1016/s1631-073x(02)02412-3>. Cited in page 38.

ALNÆES, M. et al. The fenics project version 1.5. Archive of Numerical Software, University Library Heidelberg, v. 3, 2015. Available from Internet: <http://journals.ub.uni-heidelberg.de/ index.php/ans/article/view/20553>. Cited in page 46.

BATHE, K.-J. Finite Element Procedures. [S.I.]: Prentice Hall, 1995. ISBN 0133014584. Cited in page 36.

BENDS $\varnothing \mathrm{E}, \mathrm{M}$. P. Optimal shape design as a material distribution problem. Structural Optimization, Springer Nature, v. 1, n. 4, p. 193-202, dec 1989. Available from Internet: $<$ https://doi.org/10.1007/bf01650949>. Cited 2 times in pages 38 and 39.

BENDS $\varnothing \mathrm{E}, \mathrm{M}$. P.; KIKUCHI, N. Generating optimal topologies in structural design using a homogenization method. Computer Methods in Applied Mechanics and Engineering, Elsevier BV, v. 71, n. 2, p. 197-224, 11 1988. Available from Internet: $<$ https://doi.org/10.1016/0045-7825(88)90086-2>. Cited in page 38.

BONET, J.; WOOD, R. D. Nonlinear Continuum Mechanics for Finite Element Analysis. [S.I.]: Cambridge University Press, 2008. ISBN 0521838703. Cited in page 90.

BORGNAKKE, C. B.; SONNTAG, R. E. Fundamentals of Thermodynamics. 7. ed. [S.I.]: John Wiley \& Sons, Inc, 2009. ISBN 978-0-470-04192-5. Cited in page 103.

BOURDIN, B.; CHAMBOLLE, A. Design-dependent loads in topology optimization. ESAIM: Control, Optimisation and Calculus of Variations, EDP Sciences, v. 9, p. 19-48, jan 2003. Available from Internet: <https://doi.org/10.1051/cocv:2002070>. Cited in page 38.

BRUGGI, M.; CINQUINI, C. An alternative truly-mixed formulation to solve pressure load problems in topology optimization. Computer Methods in Applied Mechanics and Engineering, Elsevier BV, v. 198, n. 17-20, p. 1500-1512, apr 2009. Available from Internet: <https://doi.org/10.1016/j.cma.2008.12.009>. Cited in page 45.

CHEN, B.-C.; KIKUCHI, N. Topology optimization with design-dependent loads. Finite Elements in Analysis and Design, Elsevier BV, v. 37, n. 1, p. 57-70, 1 2001. Available from Internet: <https://doi.org/10.1016/s0168-874x(00)00021-4>. Cited 2 times in pages 45 and 100 .

CHEN, F. et al. Topology optimized design, fabrication, and characterization of a soft cable-driven gripper. IEEE Robotics and Automation Letters, Institute of Electrical and Electronics Engineers (IEEE), v. 3, n. 3, p. 2463-2470, jul 2018. Available from Internet: $<$ https://doi.org/10.1109/Ira.2018.2800115>. Cited in page 25.

DEEPAK, S. R. et al. A comparative study of the formulations and benchmark problems for the topology optimization of compliant mechanisms. Journal of Mechanisms and Robotics, ASME International, v. 1, n. 1, p. 011003, 2009. Available from Internet: $<$ https://doi.org/10.1115/1.2959094>. Cited in page 44. 
DENG, H.; CHENG, L.; TO, A. C. Distortion energy-based topology optimization design of hyperelastic materials. Structural and Multidisciplinary Optimization, Springer Nature, dec 2018. Available from Internet: <https://doi.org/10.1007/s00158-018-2161-6>. Cited in page 25.

DU, J.; OLHOFF, N. Topological optimization of continuum structures with design-dependent surface loading - part i: new computational approach for $2 \mathrm{~d}$ problems. Structural and Multidisciplinary Optimization, Springer Nature, v. 27, n. 3, p. 151-165, 5 2004a. Available from Internet: <https://doi.org/10.1007/s00158-004-0379-y>. Cited 2 times in pages 45 and 100.

DU, J.; OLHOFF, N. Topological optimization of continuum structures with design-dependent surface loading - part II: algorithm and examples for 3d problems. Structural and Multidisciplinary Optimization, Springer Nature, v. 27, n. 3, p. 166-177, 5 2004b. Available from Internet: <https://doi.org/10.1007/s00158-004-0380-5>. Cited in page 45.

DUFF, I. S. Ma57-a code for the solution of sparse symmetric definite and indefinite systems. ACM Trans. Math. Softw., ACM, New York, NY, USA, v. 30, n. 2, p. 118-144, jun. 2004. ISSN 0098-3500. Available from Internet: <http://doi.acm.org/10.1145/992200.992202>. Cited in page 46.

FARRELL, P. E. et al. Automated derivation of the adjoint of high-level transient finite element programs. SIAM Journal on Scientific Computing, Society for Industrial \& Applied Mathematics (SIAM), v. 35, n. 4, p. C369-C393, jan 2013. Available from Internet: $<$ https://doi.org/10.1137/120873558>. Cited 2 times in pages 46 and 47.

FUNKE, S. W.; FARRELL, P. E. A framework for automated pde-constrained optimisation. CoRR, abs/1302.3894, 2013. Available from Internet: < http://arxiv.org/abs/1302.3894>. Cited 2 times in pages 46 and 47.

GALLOWAY, K. C. et al. Mechanically programmable bend radius for fiber-reinforced soft actuators. In: 2013 16th International Conference on Advanced Robotics (ICAR). IEEE, 2013. Available from Internet: <https://doi.org/10.1109/icar.2013.6766586>. Cited 3 times in pages 22, 26, and 58 .

GONG, X. et al. Rotary actuators based on pneumatically driven elastomeric structures. Advanced Materials, Wiley, v. 28, n. 34, p. 7533-7538, jun. 2016. Available from Internet: $<$ https://doi.org/10.1002/adma.201600660>. Cited in page 23.

GUEST, J. K.; PRÉVOST, J. H.; BELYTSCHKO, T. Achieving minimum length scale in topology optimization using nodal design variables and projection functions. International Journal for Numerical Methods in Engineering, Wiley-Blackwell, v. 61, n. 2, p. 238-254, 8 2004. Available from Internet: <https://doi.org/10.1002/nme.1064>. Cited in page 40.

GüNNEL, A. Numerical Aspects in Optimal Control of Elasticity Models with Large Deformations. Tese (Doutorado) - Faculty of Mathematics at Chemnitz University of Technology, 2014. Cited in page 91.

HAMMER, V.; OLHOFF, N. Topology optimization of continuum structures subjected to pressure loading. Structural and Multidisciplinary Optimization, Springer Nature, v. 19, n. 2, p. 85-92, 4 2000. Available from Internet: <https://doi.org/10.1007/s001580050088>. Cited 2 times in pages 45 and 100. 
HILLER, J.; LIPSON, H. Automatic design and manufacture of soft robots. IEEE Transactions on Robotics, Institute of Electrical and Electronics Engineers (IEEE), v. 28, n. 2, p. 457-466, apr 2012. Available from Internet: <https://doi.org/10.1109/tro.2011.2172702>. Cited 2 times in pages 25 and 26.

LAI, W. M.; RUBIN, D.; KREMPL, E. Introduction to Continuum Mechanics. [S.I.]: Elsevier, 2009. ISBN 9780750685603. Cited 2 times in pages 27 and 59.

LASCHI, C.; MAZZOLAI, B.; CIANCHETTI, M. Soft robotics: Technologies and systems pushing the boundaries of robot abilities. Science Robotics, American Association for the Advancement of Science (AAAS), v. 1, n. 1, p. eaah3690, nov. 2016. Available from Internet: $<$ https://doi.org/10.1126/scirobotics.aah3690>. Cited 2 times in pages 10 and 22.

LAZAROV, B. S.; SIGMUND, O. Filters in topology optimization based on helmholtz-type differential equations. International Journal for Numerical Methods in Engineering, Wiley-Blackwell, v. 86, n. 6, p. 765-781, 12 2010. Available from Internet: < https: //doi.org/10.1002/nme.3072>. Cited 2 times in pages 25 and 40.

LOGG, A. et al. Automated Solution of Differential Equations by the Finite Element Method. [S.I.]: Springer, 2012. ISBN 978-3-642-23098-1. Cited in page 47.

MASE, G. T.; MASE, G. E. Continuum mechanics for engineers. 2. ed. [S.I.]: CRC Press LLC, 1999. ISBN 0849318556. Cited in page 27.

MIDHA, A.; NORTON, T. W.; HOWELL, L. L. On the nomenclature, classification, and abstractions of compliant mechanisms. Journal of Mechanical Design, ASME International, v. 116, n. 1, p. 270, 1994. Available from Internet: <https://doi.org/10.1115/1.2919358>. Cited in page 44.

MOSADEGH, B. et al. Pneumatic networks for soft robotics that actuate rapidly. Advanced Functional Materials, Wiley-Blackwell, v. 24, n. 15, p. 2163-2170, 1 2014. Available from Internet: <https://doi.org/10.1002/adfm.201303288>. Cited 3 times in pages 22, 26, and 58.

PARK, Y.-L. et al. Hyperelastic pressure sensing with a liquid-embedded elastomer. Journal of Micromechanics and Microengineering, IOP Publishing, v. 20, n. 12, p. 125029, 112010. Available from Internet: < https://doi.org/10.1088/0960-1317/20/12/125029>. Cited in page 23.

PEDERSEN, C. B. W.; BUHL, T.; SIGMUND, O. Topology synthesis of large-displacement compliant mechanisms. International Journal for Numerical Methods in Engineering, Wiley-Blackwell, v. 50, n. 12, p. 2683-2705, 2001. Available from Internet: < https: //doi.org/10.1002/nme.148>. Cited 3 times in pages 44, 56, and 65.

PENCE, T. J.; GOU, K. On compressible versions of the incompressible neo-hookean material. Mathematics and Mechanics of Solids, SAGE Publications, v. 20, n. 2, p. 157-182, ago. 2014. Available from Internet: <https://doi.org/10.1177/1081286514544258>. Cited in page 91.

PETERSEN, K. B.; PEDERSEN, M. S. The Matrix Cookbook. [S.I.: s.n.], 2012. Cited in page 91.

ROJAS-LABANDA, S.; STOLPE, M. Benchmarking optimization solvers for structural topology optimization. Structural and Multidisciplinary Optimization, Springer Science and Business Media LLC, v. 52, n. 3, p. 527-547, maio 2015. Available from Internet: $<$ https://doi.org/10.1007/s00158-015-1250-z>. Cited in page 47. 
ROTHEMUND, P. et al. A soft, bistable valve for autonomous control of soft actuators.

Science Robotics, American Association for the Advancement of Science (AAAS), v. 3, n. 16, p. eaar7986, mar. 2018. Available from Internet: <https://doi.org/10.1126/scirobotics.aar7986>. Cited in page 23.

SIGMUND, O. Morphology-based black and white filters for topology optimization. Structural and Multidisciplinary Optimization, Springer Nature, v. 33, n. 4-5, p. 401-424, 12007. Available from Internet: <https://doi.org/10.1007/s00158-006-0087-x>. Cited 2 times in pages 40 and 41 .

SIGMUND, O.; CLAUSEN, P. Topology optimization using a mixed formulation: An alternative way to solve pressure load problems. Computer Methods in Applied Mechanics and Engineering, Elsevier BV, v. 196, n. 13-16, p. 1874-1889, 3 2007. Available from Internet: <https://doi.org/10.1016/j.cma.2006.09.021>. Cited 5 times in pages 25, 45, 94, 95, and 100 .

SIGMUND, O.; MAUTE, K. Topology optimization approaches. Structural and Multidisciplinary Optimization, Springer Nature, v. 48, n. 6, p. 1031-1055, 8 2013. Available from Internet: $<$ https://doi.org/10.1007/s00158-013-0978-6>. Cited 2 times in pages 39 and 61.

SIGMUND, O.; PETERSSON, J. Numerical instabilities in topology optimization: A survey on procedures dealing with checkerboards, mesh-dependencies and local minima. Structural Optimization, Springer Science and Business Media LLC, v. 16, n. 1, p. 68-75, aug 1998. Available from Internet: <https://doi.org/10.1007/bf01214002>. Cited in page 42.

SO, J.-H. et al. Reversibly deformable and mechanically tunable fluidic antennas. Advanced Functional Materials, Wiley-Blackwell, v. 19, n. 22, p. 3632-3637, 11 2009. Available from Internet: <https://doi.org/10.1002/adfm.200900604>. Cited in page 23.

SOKOLOWSKI, J.; ZOCHOWSKI, A. On the topological derivative in shape optimization. SIAM Journal on Control and Optimization, Society for Industrial \& Applied Mathematics (SIAM), v. 37, n. 4, p. 1251-1272, jan 1999. Available from Internet: $<$ https://doi.org/10.1137/s0363012997323230>. Cited in page 38.

SONG, H. Engineering Fluid Mechanics. Springer Singapore, 2018. Available from Internet: $<$ https://doi.org/10.1007/978-981-13-0173-5>. Cited in page 59.

SOUZA, E. M. de; SILVA, E. C. N. Topology optimization applied to the design of actuators driven by pressure loads. Structural and Multidisciplinary Optimization, Springer Science and Business Media LLC, mar. 2020. Available from Internet: $<$ https://doi.org/10.1007/s00158-019-02421-5>. Cited 3 times in pages 27, 42, and 79.

SUZUMORI, K. Elastic materials producing compliant robots. Robotics and Autonomous Systems, Elsevier BV, v. 18, n. 1-2, p. 135-140, 7 1996. Available from Internet: <https://doi.org/10.1016/0921-8890(95)00078-x>. Cited 2 times in pages 21 and 58.

TRIMMER, B. A practical approach to soft actuation. Soft Robotics, Mary Ann Liebert Inc, v. 4, n. 1, p. 1-2, 3 2017. Available from Internet: <https://doi.org/10.1089/soro.2017.29011.bat>. Cited in page 26.

WäCHTER, A. Short Tutorial: Getting Started With Ipopt in 90 Minutes. 2009. Cited in page 47. 
WäCHTER, A.; BIEGLER, L. T. On the implementation of an interior-point filter line-search algorithm for large-scale nonlinear programming. Mathematical Programming, Springer Nature, v. 106, n. 1, p. 25-57, apr 2005. Available from Internet: $<$ https://doi.org/10.1007/s10107-004-0559-y>. Cited 3 times in pages 46, 47, and 48.

WANG, F.; LAZAROV, B. S.; SIGMUND, O. On projection methods, convergence and robust formulations in topology optimization. Structural and Multidisciplinary Optimization, Springer Nature, v. 43, n. 6, p. 767-784, dec 2010. Available from Internet: $<$ https://doi.org/10.1007/s00158-010-0602-y>. Cited 3 times in pages 25, 40, and 41.

XIE, Y.; STEVEN, G. A simple evolutionary procedure for structural optimization. Computers \& Structures, Elsevier BV, v. 49, n. 5, p. 885-896, dec 1993. Available from Internet: $<$ https://doi.org/10.1016/0045-7949(93)90035-c >. Cited in page 38.

XU, S.; CAI, Y.; CHENG, G. Volume preserving nonlinear density filter based on heaviside functions. Structural and Multidisciplinary Optimization, Springer Science and Business Media LLC, v. 41, n. 4, p. 495-505, dez. 2009. Available from Internet: $<$ https://doi.org/10.1007/s00158-009-0452-7>. Cited in page 40.

ZHANG, H. et al. Design and development of a topology-optimized three-dimensional printed soft gripper. Soft Robotics, Mary Ann Liebert Inc, v. 5, n. 5, p. 650-661, out. 2018. Available from Internet: <https://doi.org/10.1089/soro.2017.0058>. Cited in page 25.

ZHAO, H. et al. Optoelectronically innervated soft prosthetic hand via stretchable optical waveguides. Science Robotics, American Association for the Advancement of Science (AAAS), v. 1, n. 1, p. eaai7529, nov. 2016. Available from Internet: $<$ https://doi.org/10.1126/scirobotics.aai7529>. Cited in page 23.

ZIENKIEWICZ, O. C.; TAYLOR, R. L. Finite Element Method: Volume 1, Fifth Edition (Finite Element Method Ser). Butterworth-Heinemann, 2000. ISBN 9780750650496. Available from Internet: <https://www.xarg.org/ref/a/0750650494/>. Cited in page 35. 


\section{APPENDIX A - RELATION BETWEEN $K$, $E_{Y}$ AND $\nu$}

The bulk modulus $(K)$ is the material property that measures the resistance of the material to isostatic compression. By considering a cube (in three dimensions - 3D) or a square (in two dimensions - 2D) subjected to compressive surface loads of magnitude $p$ on all its surfaces, the bulk modulus is defined by the relation between $p$ and the dilatation $e$.

$$
K=-\frac{p}{e}
$$

The dilatation can be expressed in terms of strain components according to Equation A.2.

$$
e=\epsilon_{11}+\epsilon_{22}+\epsilon_{33}
$$

To write the bulk modulus in terms of Young's modulus $E_{Y}$ and Poisson's ratio $\nu$ by using Equation A.2, first it is necessary to write the strain tensor $\epsilon$ in terms of $E_{Y}$ and $\nu$. Equation A.3 expresses all strain components in terms of stress components, $E_{Y}$ and $\nu$.

$$
\boldsymbol{\epsilon}=\frac{1}{E_{Y}}[(1+\nu) \boldsymbol{\sigma}-\nu \operatorname{tr}(\boldsymbol{\sigma}) \boldsymbol{I}]
$$

According to Equation A.3, the normal strain components are given by Equation A.4.

$$
\begin{aligned}
& \epsilon_{11}=\frac{1}{E_{Y}}\left(\sigma_{11}-\nu \sigma_{22}-\nu \sigma_{33}\right) \\
& \epsilon_{22}=\frac{1}{E_{Y}}\left(-\nu \sigma_{11}+\sigma_{22}-\nu \sigma_{33}\right) \\
& \epsilon_{33}=\frac{1}{E_{Y}}\left(-\nu \sigma_{11}-\nu \sigma_{22}+\sigma_{33}\right)
\end{aligned}
$$

By substituting Equation A.4 in Equation A.2, the general relation between dilatation and normal stress components is obtained in Equation A.5.

$$
e=\frac{(1-2 \nu)}{E_{Y}}\left(\sigma_{11}+\sigma_{22}+\sigma_{33}\right)
$$

According to Equation A.5, the dilatation and, therefore, the bulk modulus depend on the number of dimensions and the strain assumption. 


\section{A.1 3D}

In 3D the stress components are all equal to the applied compressive pressure $p$ according to Equation A.6.

$$
\sigma_{11}=\sigma_{22}=\sigma_{33}=-p
$$

By substituting Equation A.6 in Equation A.5, the dilatation for three dimensional models is obtained according to Equation A.7.

$$
e=-3 p \frac{(1-2 \nu)}{E_{Y}}
$$

The bulk modulus for 3D models is obtained by substituting Equation A.7 in Equation A.1 and the result is Equation A.8.

$$
K=\frac{E_{Y}}{3(1-2 \nu)}
$$

\section{A.2 2D Plane Stress}

In 2D plane stress, the stress component on the axis perpendicular to the plane is zero $\left(\sigma_{33}=0\right)$ while the stress components on the plane are equal to the compressive pressure $p$ according to Equation A.9.

$$
\begin{aligned}
\sigma_{11} & =\sigma_{22}=-p \\
\sigma_{33} & =0
\end{aligned}
$$

The dilatation is obtained by substituting Equation A.9 in Equation A.5 and the result is Equation A.10.

$$
e=-2 p \frac{(1-2 \nu)}{E_{Y}}
$$

The bulk modulus is then obtained by substituting Equation A.10 in Equation A.1 and the result is Equation A.11.

$$
K=\frac{E_{Y}}{2(1-2 \nu)}
$$




\section{A.3 2D Plane Strain}

In 2D plane strain, there is no deformation in the perpendicular direction of the plane $\left(\epsilon_{33}\right.$ $=0)$. So, according to Equation A.4, the stress component $\sigma_{33}$ can be expressed in terms of $\sigma_{11}$ and $\sigma_{22}$ and the compressive stress terms are given by Equation A.12.

$$
\begin{aligned}
& \sigma_{11}=\sigma_{22}=-p \\
& \sigma_{33}=\nu\left(\sigma_{11}+\sigma_{22}\right)=-2 \nu p
\end{aligned}
$$

The dilatation is obtained by substituting Equation A.13 in Equation A.5 and the result is Equation A.10.

$$
e=-2 p \frac{(1-2 \nu)(1+\nu)}{E_{Y}}
$$

Then, the bulk modulus is obtained by substituting the dilatation of Equation A.13 in Equation A.1.

$$
K=\frac{E_{Y}}{2(1-2 \nu)(1+\nu)}
$$




\section{APPENDIX B - NONLINEAR MATERIAL MODEL}

\section{B.1 Hyperelastic Material}

A hyperelastic material is characterized by a scalar strain energy density function $W$ which describes the stress-strain relationship according to Equation B.1 where $\boldsymbol{S}$ is the second Piola-Kirchhoff stress tensor and $\boldsymbol{E}$ is the Green-Lagrange strain tensor.

$$
S=\frac{\partial W}{\partial E}
$$

The static equilibrium of a hyperelastic body $\Omega$ can be formulated as to find the displacement field $\boldsymbol{u}$ that minimizes the total potential energy $\Pi$ according to Equation B.2.

$$
\min _{\boldsymbol{u}} \Pi(\boldsymbol{u})
$$

The total potential energy $\Pi$ is calculated by considering the energy of the whole body due to strain, the work of the body forces $b$ and the work of traction forces $t$ according to Equation B.3.

$$
\Pi(\boldsymbol{u})=\int_{\Omega} W(\boldsymbol{u}) d \Omega-\int_{\Omega} \boldsymbol{b} \cdot \boldsymbol{u} d \Omega-\int_{\Gamma} \boldsymbol{t} \cdot \boldsymbol{u} d \Gamma
$$

At minimum points of $\Pi$, the directional derivative of $\Pi$ with respect to $\boldsymbol{u}$ in the direction $\boldsymbol{\delta} \boldsymbol{u}$ (Equation B.4) must be equal to zero for any direction $\boldsymbol{\delta} \boldsymbol{u}$ according to Equation B.5.

$$
\begin{gathered}
L(\boldsymbol{u} ; \boldsymbol{\delta} \boldsymbol{u})=D_{\delta \boldsymbol{u}} \Pi=\lim _{\epsilon \rightarrow 0} \frac{\Pi(\boldsymbol{u}+\epsilon \boldsymbol{\delta} \boldsymbol{u})-\Pi(\boldsymbol{u})}{\epsilon}=\frac{\partial \Pi}{\boldsymbol{\partial u}} \cdot \boldsymbol{\delta} \boldsymbol{u} \\
L(\boldsymbol{u} ; \boldsymbol{\delta} \boldsymbol{u})=0
\end{gathered}
$$

By using Equation B.3 in B.4 and by calculating the limit, Equation B.6 is obtained.

$$
L(\boldsymbol{u} ; \boldsymbol{\delta} \boldsymbol{u})=\int_{\Omega} D_{\delta \boldsymbol{u}} W(\boldsymbol{u}) d \Omega-\int_{\Omega} \boldsymbol{b} \cdot \boldsymbol{\delta} \boldsymbol{u} d \Omega-\int_{\Gamma} \boldsymbol{t} \cdot \boldsymbol{\delta} \boldsymbol{u} d \Gamma
$$

In general, it is easier to calculate the partial derivative of $W$ with respect to $\boldsymbol{F}$ than the 
partial derivative of $W$ with respect to $\boldsymbol{u}$, so the Equation C.6 is used to rewrite Equation B.6 and the result is present in Equation B.7.

$$
L(\boldsymbol{u} ; \boldsymbol{\delta} \boldsymbol{u})=\int_{\Omega} D_{\nabla \boldsymbol{\delta} \boldsymbol{u}} W(\boldsymbol{F}) d \Omega-\int_{\Omega} \boldsymbol{b} \cdot \boldsymbol{\delta} \boldsymbol{u} d \Omega-\int_{\Gamma} \boldsymbol{t} \cdot \boldsymbol{\delta} \boldsymbol{u} d \Gamma
$$

As the energy density function $W(\boldsymbol{F})$ is expected to be nonlinear, the Jacobian of $L$ is required for solving Equation B.5 by Newton's Method. The Jacobian is calculated by taking the directional derivative of $L$ in direction $\boldsymbol{d u}$ according to Equation B.8.

$$
a(\boldsymbol{u} ; \boldsymbol{d u}, \boldsymbol{\delta} \boldsymbol{u})=D_{\boldsymbol{d u}} L(\boldsymbol{u} ; \boldsymbol{\delta} \boldsymbol{u})=\lim _{\epsilon \rightarrow 0} \frac{L(\boldsymbol{u}+\epsilon \boldsymbol{d u} ; \boldsymbol{\delta} \boldsymbol{u})-L(\boldsymbol{u} ; \boldsymbol{\delta} \boldsymbol{u})}{\epsilon}=\frac{\partial L}{\boldsymbol{\partial u}} \cdot \boldsymbol{d u}
$$

As $W$ is now expressed as a function of $\boldsymbol{F}$, Equation C.6 is used again for changing variable in directional derivative and Equation B.9 is obtained.

$$
a(\boldsymbol{u} ; \boldsymbol{d u}, \boldsymbol{\delta} \boldsymbol{u})=\int_{\Omega} D_{\boldsymbol{\nabla} \boldsymbol{d u}} D_{\boldsymbol{\nabla} \delta \boldsymbol{u}} W(\boldsymbol{F}) d \Omega
$$

There are several hyperelastic models such as Neo-Hookean, Mooney-Rivlin, Ogden, and Arruda-Boyce models. This work uses the Neo-Hookean model to check if the obtained results also deforms in desired direction when nonlinear models are considered.

\section{B.1.1 Neo-Hookean Solid}

One of the equations that can be used to describe a Neo-Hookean material is presented in Equation B.10 (BONET; WOOD, 2008), where $C_{1}$ and $D_{1}$ are materials constants to be determined experimentally. The scalar $I_{1}$ is the first invariant of right Cauchy-Green tensor $I_{1}$ $=\operatorname{Tr}(\boldsymbol{C})=\operatorname{Tr}\left(\boldsymbol{F}^{T} \boldsymbol{F}\right)$.

$$
W= \begin{cases}C_{1}\left(I_{1}-2-2 \ln (J)\right)+D_{1}(\ln (J))^{2} & \text { for } 2 \mathrm{D} \text { problems } \\ C_{1}\left(I_{1}-3-2 \ln (J)\right)+D_{1}(\ln (J))^{2} & \text { for 3D problems }\end{cases}
$$

For an incompressible material, $J=1$ and $\ln (J)=0$. Therefore, the Neo-Hookean energy density function is reduced to Equation B.11 where just one material constant $C_{1}$ is required to describe material behavior.

$$
W= \begin{cases}C_{1}\left(I_{1}-2\right) & \text { for } 2 \mathrm{D} \text { problems } \\ C_{1}\left(I_{1}-3\right) & \text { for } 3 \mathrm{D} \text { problems }\end{cases}
$$


By considering the compressible 2D case, the Neo-Hookean material strain energy density function can be written in terms of the deformation gradient $\boldsymbol{F}$ according to Equation B.12. The material constants $C_{1}=\mu / 2$ and $D_{1}=\lambda / 2$ are chosen and they guarantee compatibility with an isotropic linearly elastic solid under small deformations (PENCE; GOU, 2014).

$$
W(\boldsymbol{F})=\frac{\mu}{2}\left(\operatorname{Tr}\left(\boldsymbol{F}^{T} \boldsymbol{F}\right)-2-2 \ln (\operatorname{det}(\boldsymbol{F}))\right)+\frac{\lambda}{2}(\ln (\operatorname{det}(\boldsymbol{F})))^{2}
$$

Therefore, the directional derivative of the strain energy density function $W$ with respect to the deformation gradient $\boldsymbol{F}$ in the direction $\boldsymbol{G}=\boldsymbol{\nabla} \boldsymbol{\delta} \boldsymbol{u}$ is given by Equation B.13 where $\boldsymbol{F}^{-T}=\left(\boldsymbol{F}^{-1}\right)^{T}=\left(\boldsymbol{F}^{T}\right)^{-1}$.

$$
D_{\boldsymbol{G}} W(\boldsymbol{F})=\frac{\partial W(\boldsymbol{F})}{\partial \boldsymbol{F}}: \boldsymbol{G}=\left(\mu \boldsymbol{F}-\mu \boldsymbol{F}^{-T}+\lambda \ln (\operatorname{det}(\boldsymbol{F})) \boldsymbol{F}^{-T}\right): \boldsymbol{G}
$$

And the directional derivative of the function $D_{\boldsymbol{G}} W(\boldsymbol{F})$ with respect to the deformation gradient $\boldsymbol{F}$ in the direction $\boldsymbol{H}=\boldsymbol{\nabla} \boldsymbol{d} \boldsymbol{u}$ is given by Equation B.14.

$$
\begin{aligned}
D_{\boldsymbol{H}}\left(D_{\boldsymbol{G}} W(\boldsymbol{F})\right)=\frac{\partial\left(D_{\boldsymbol{G}} W(\boldsymbol{F})\right)}{\boldsymbol{\partial} \boldsymbol{F}}: \boldsymbol{H}= \\
\quad\left(\mu \boldsymbol{G}+\mu \boldsymbol{F}^{-T} \boldsymbol{G F}^{-T}+\lambda \boldsymbol{F}^{-T}\left(\boldsymbol{F}^{-T}: \boldsymbol{G}\right)-\lambda \ln (\operatorname{det}(\boldsymbol{F})) \boldsymbol{F}^{-T} \boldsymbol{G} \boldsymbol{F}^{-T}\right): \boldsymbol{H}
\end{aligned}
$$

More details about matrix derivatives can be found in Petersen and Pedersen (2012) and about differentiation of strain energy density functions in Günnel (2014).

\section{B.2 Newton's Method}

As Equation B.5 is nonlinear, it is necessary to use a nonlinear solver algorithm such as Newton's method.

In Newton's method, the step of iteration $(k+1)$ is given by Equation B.15 in which $\Delta \boldsymbol{u}_{k}$ is the search direction and $\alpha>0$ is the step length. For simplicity, this work uses $\alpha=1$, since the interest is just to check the optimized results under large deformations, so a load that guarantees convergence can be chosen.

$$
\boldsymbol{u}_{k+1}=\boldsymbol{u}_{k}+\alpha \Delta \boldsymbol{u}_{k}
$$

The search direction $\Delta \boldsymbol{u}_{k}$ is found by solving the linear system presented by Equation 
B.16.

$$
a\left(\boldsymbol{u} ; \Delta \boldsymbol{u}_{k}, \boldsymbol{\delta} \boldsymbol{u}\right)=-L(\boldsymbol{u} ; \boldsymbol{\delta} \boldsymbol{u})
$$




\section{APPENDIX C - DERIVATIVES OF STRAIN ENERGY DENSITY FUNCTIONS}

The derivative of some strain energy density functions $W$ are more easily calculated when $W$ is expressed in terms of the deformation gradient $\boldsymbol{F}$ instead of the displacement $\boldsymbol{u}$. The directional derivative of $W(\boldsymbol{u})$ in the direction of $\boldsymbol{\delta} \boldsymbol{u}$ is expressed in Einstein notation by Equation C.1.

$$
D_{\boldsymbol{\delta} \boldsymbol{u}} W(\boldsymbol{u})=\frac{\partial W}{\partial u_{i}} \delta u_{i}
$$

By expanding the directional derivative of $W$ with respect to $u_{i}$, it is possible express it in terms of the deformation gradient $F_{j k}$ according to Equation C.2.

$$
\frac{\partial W}{\partial u_{i}} \delta u_{i}=\frac{\partial W}{\partial F_{j k}} \frac{\partial F_{j k}}{\partial u_{i}} \delta u_{i}
$$

The derivative of the deformation gradient $F_{j k}$ with respect to the displacement $u_{i}$ is obtained from the deformation gradient $F_{j k}$ expression according to Equation C.3 where $\delta_{j k}$ is the identity matrix.

$$
F_{j k}=\delta_{j k}+\frac{\partial u_{k}}{\partial x_{j}} \Rightarrow \frac{\partial F_{j k}}{\partial u_{i}}=\frac{\partial u_{k}}{\partial u_{i} \partial x_{j}}=\frac{\partial \delta_{i k}}{\partial x_{j}}
$$

In Equation C.2, the derivative of $F_{j k}$ with respect to $u_{i}$ appears multiplied by the test function $\delta u_{i}$. The combination of these terms can be rearranged according to C.4 to reduce C. 2 to C.5.

$$
\begin{gathered}
\frac{\partial F_{j k}}{\partial u_{i}} \delta u_{i}=\frac{\partial \delta_{i k}}{\partial x_{j}} \delta u_{i}=\frac{\partial \delta u_{k}}{\partial x_{j}} \\
\frac{\partial W}{\partial u_{i}} \delta u_{i}=\frac{\partial W}{\partial F_{j k}} \frac{\partial \delta u_{k}}{\partial x_{j}}
\end{gathered}
$$

Therefore, the directional derivative of the strain energy density function can also be calculated by Equation C.6.

$$
D_{\boldsymbol{\delta} \boldsymbol{u}} W(\boldsymbol{u})=D_{\boldsymbol{\nabla} \boldsymbol{\delta} \boldsymbol{u}} W(\boldsymbol{F})=\frac{\partial W}{\boldsymbol{\partial} \boldsymbol{F}}: \boldsymbol{\nabla} \boldsymbol{\delta} \boldsymbol{u}
$$




\section{APPENDIX D - IMPLEMENTATION IN FENICS}

The evaluation of the optimization routines is made with two examples extracted from Sigmund and Clausen (2007): the bridge and the internally pressurized lid. The approach followed is to compare the results obtained with the mixed model against the results obtained with a standard displacement model according to what is done in the paper (SIGMUND; CLAUSEN, 2007) of the examples. A direct comparison to literature results is not made because the filters used are different and no length control methods are employed.

Although the problems explored in this appendix use minimum compliance formulations instead of compliant mechanisms formulations that are intended to be used for pressure-driven actuators design, the problems are still useful for forward problem and topology optimization loop validation. The objective function $J_{C}$ used in this chapter is the work of traction forces $t$ which is given by Equation D.1.

$$
J_{C}(\boldsymbol{u})=\int_{\Gamma} \boldsymbol{t} \cdot \boldsymbol{u} d \Gamma
$$

The continuation update rules used in this chapter for penalization parameter $z$ and threshold projection parameter $\beta$ are presented in Equation D.2.

$$
\begin{aligned}
& z_{i+1}=z_{i}+1 \\
& \beta_{i+1}=2 \beta_{i}
\end{aligned}
$$

The termination criteria of IPOPT used in the examples of this appendix are desired convergence tolerance $\left(\epsilon_{t o l}\right)$ and maximum number of iterations $\left(n_{\max }\right)$. The selected values are presented in Equation D.3. The same values of $\epsilon_{t o l}$ and $n_{\max }$ are used in all continuation steps.

$$
\begin{aligned}
& \epsilon_{\text {tol }}=10^{-3} \\
& n_{\text {max }}=100
\end{aligned}
$$

\section{D.1 Bridge example}

The first example solved by using mixed formulation is the bridge from Sigmund and Clausen (2007) which considers solid and void phases. The design domain is a rectangle of dimensions $L$ and $H$ according to Figure D.1. The upper part of design domain which has 
height $H_{s}$ is fixed to be solid. The fixed solid region is indicated by black color in Figure D.1. The dimensions used for the bridge example are presented in Equation D.4.

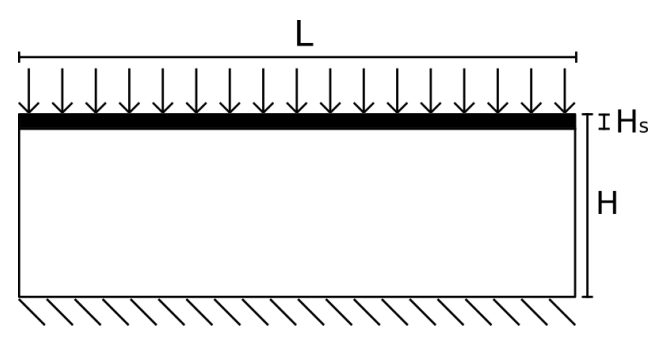

Figure D.1 - Design domain of bridge example. The region in black is fixed to be solid.

$$
\begin{aligned}
& L=1 \\
& H=1 / 3 \\
& H_{s}=H / 10
\end{aligned}
$$

The problem is discretized by FEniCS' right triangular elements of size is 0.01 . The interpolation functions used are Lagrange functions of degree two for displacement field and of degree one for pressure and design variable fields. It is necessary to use functions of degree one for design variables due to the density filter.

The mesh is composed of 6600 finite elements and the optimization problem has 3030 design variables.

The material properties used are the same as Sigmund and Clausen (2007), but the solid Poisson's ratio is set to $\nu_{s}=0.4$ to obtain a behavior closer to elastomers. The void phase is modeled by considering small values for $K_{f}$ and $G_{v}$ according to guidelines of Equation 2.71. The material properties used in this appendix are listed in Equation D.5.

$$
\begin{array}{ll}
E_{s}=1 & \nu_{s}=0.4 \quad \text { (plane stress) } \\
K_{f}=10^{-6} & G_{v}=10^{-6}
\end{array}
$$

The pressure load is applied over the fixed solid region according to D.1. As the load surface does not change during the optimization, it is equivalent to a fixed direction traction load, so the pure displacement formulation can be executed without worrying about the pressure load. The value used for $\boldsymbol{t}$ is given in Equation D.6.

$$
\boldsymbol{t}=\left[\begin{array}{c}
0 \\
-1
\end{array}\right]
$$

The allowed material volume $V^{*}$ (or volume constraint) of bridge example is $30 \%$. By 
considering a unit depth, the value of $V^{*}$ is given by Equation D.7.

$$
V^{*}=0.3 L H
$$

The density filter length parameter $r$ and threshold projection parameter $\eta$ used are presented in Equation D.8. It is worth recalling that the density filter and threshold projection do not guarantee minimum length size, because it is necessary to select $\eta=0$ for minimum length scale at solid phase and $\eta=1$ for minimum length scale at void or fluid phase.

$$
\begin{aligned}
& r=1.5 \\
& \eta=0.5
\end{aligned}
$$

\section{D.1.1 Comparison to standard displacement formulation}

The topology optimization results of bridge example with standard displacement formulation and mixed displacement-pressure formulation are presented in Figure D.2. Both results have the same topological features: three vertical columns with $\mathrm{Y}$ shape supporting the fixed solid region. The sizing of components is slightly different as expected because the finite element discretization is different. The differences in FEM discretization cause small differences in results.

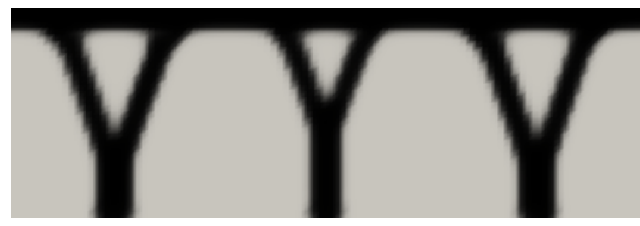

(a) Standard displacement topology

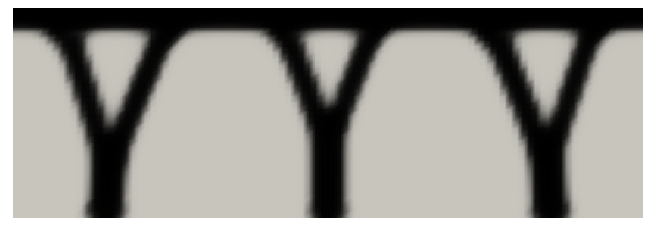

(b) Mixed formulation topology

Figure D.2 - Results for bridge example by considering standard displacement and mixed formulation.

\section{D.1.2 SIMP}

The first study made with the bridge example is to optimize for different values of penalization parameter $z$ while keeping the threshold projection parameter $\beta$ fixed in $\beta=$ 1. This is equivalent to not applying threshold projection. The idea is to verify the effect of changing $z$. The results are presented in Figure D.3 and Table D.1. From the discreteness $M_{n d}$ values presented in Table D.1, it is possible to verify the expected penalization of intermediate densities for the minimum compliance problem. 


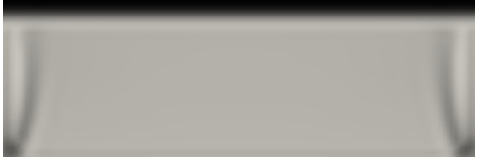

(a) $z=1$

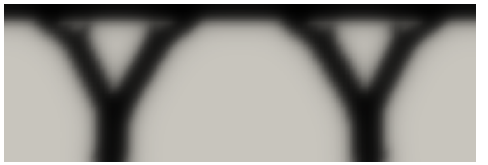

(d) $z=4$

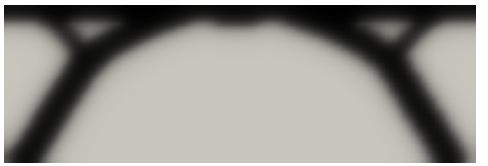

(g) $z=7$

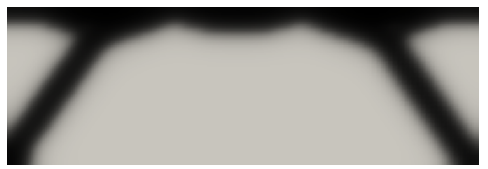

(j) $z=10$

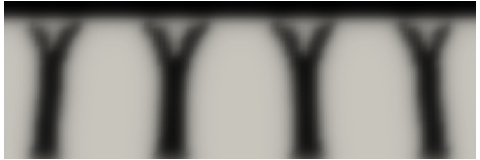

(b) $z=2$

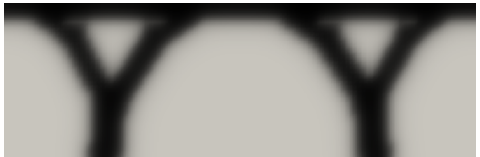

(e) $z=5$

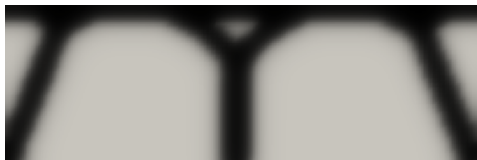

(h) $z=8$

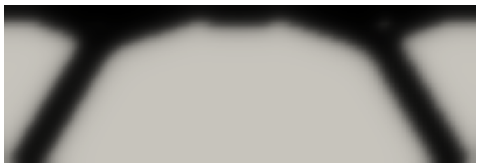

(k) $z=11$

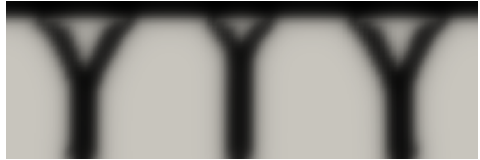

(c) $z=3$

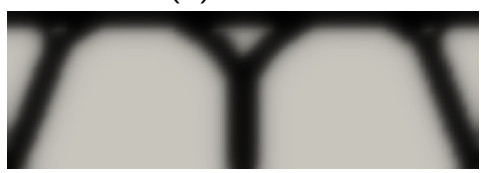

(f) $z=6$

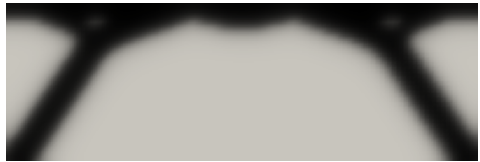

(i) $z=9$

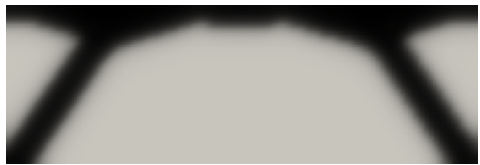

(I) $z=12$

Figure D.3 - Bridge topologies by considering different penalization parameters (see Table D.1).

Table D.1 - Bridge results using different penalization parameters.

\begin{tabular}{cccc}
\hline Reference & $\mathrm{z}$ & $J_{C}$ & $M_{n d}(\%)$ \\
\hline Figure D.3a & 1 & 1.19 & 69.7 \\
Figure D.3b & 2 & 2.68 & 42.2 \\
Figure D.3c & 3 & 4.17 & 37.0 \\
Figure D.3d & 4 & 6.72 & 33.4 \\
Figure D.3e & 5 & 8.00 & 30.6 \\
Figure D.3f & 6 & 9.37 & 31.1 \\
Figure D.3g & 7 & 22.1 & 30.0 \\
Figure D.3h & 8 & 13.3 & 30.0 \\
Figure D.3i & 9 & 31.5 & 26.3 \\
Figure D.3j & 10 & 53.4 & 32.1 \\
Figure D.3k & 11 & 38.0 & 27.6 \\
Figure D.3l & 12 & 41.9 & 24.9
\end{tabular}

\section{D.1.3 SIMP with threshold projection continuation}

The bridge example is also solved by using threshold projection after density filtering and the results are presented in Figure D.4 and Table D.2. The threshold parameter $\beta$ is initialized with $\beta_{\min }=1$ and the continuation is made until $\beta_{\max }=8$ while the penalization parameter $z$ is kept constant. The comparison of discreteness $M_{n d}$ from Tables D.1 and D.4 shows that threshold projection reduces the gray transition regions. 


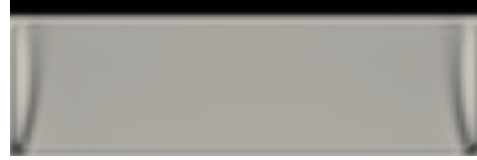

(a) $z=1$

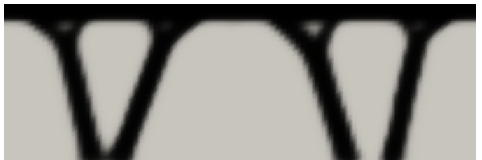

(d) $z=4$

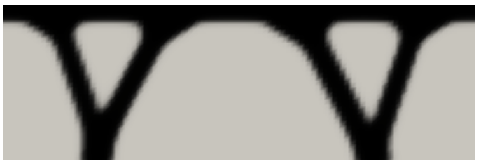

(g) $z=7$

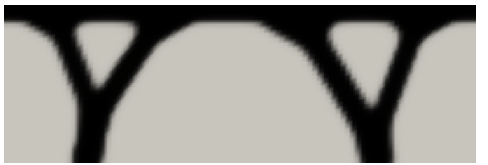

(j) $z=10$

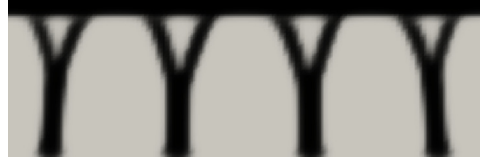

(b) $z=2$

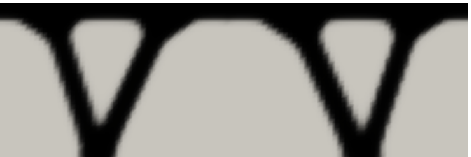

(e) $z=5$

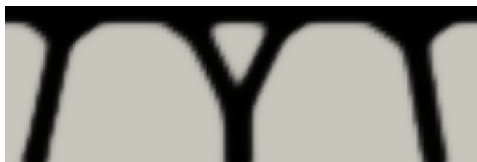

(h) $z=8$

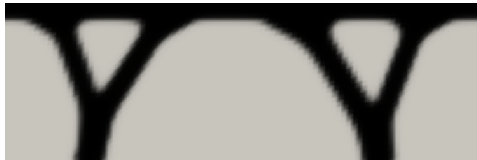

(k) $z=11$

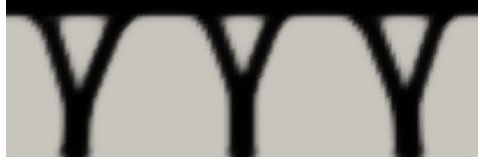

(c) $z=3$

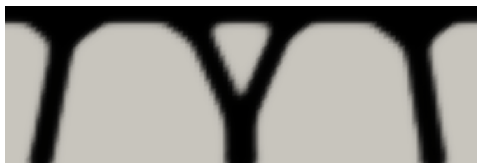

(f) $z=6$

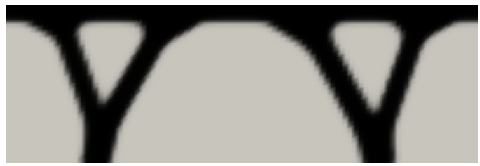

(i) $z=9$

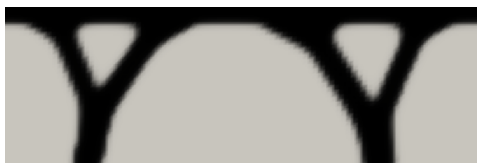

(I) $z=12$

Figure D.4 - Bridge topologies by considering different penalization parameters and continuation of threshold projection from $\beta_{\min }=1$ to $\beta_{\max }=8$ (see Table D.2).

Table D.2 - Bridge results by using different penalization parameters and continuation of threshold projection from $\beta_{\min }=1$ to $\beta_{\max }=8$.

\begin{tabular}{cccc}
\hline Reference & $\mathrm{z}$ & $J_{C}$ & $M_{n d}(\%)$ \\
\hline Figure D.4a & 1 & 1.29 & 59.7 \\
Figure D.4b & 2 & 2.12 & 14.7 \\
Figure D.4c & 3 & 2.60 & 11.7 \\
Figure D.4d & 4 & 3.54 & 13.1 \\
Figure D.4e & 5 & 3.86 & 9.0 \\
Figure D.4f & 6 & 3.55 & 9.6 \\
Figure D.4g & 7 & 4.36 & 9.0 \\
Figure D.4h & 8 & 3.99 & 9.9 \\
Figure D.4i & 9 & 4.67 & 8.5 \\
Figure D.4j & 10 & 4.99 & 8.9 \\
Figure D.4k & 11 & 5.13 & 9.1 \\
Figure D.4l & 12 & 5.19 & 8.2
\end{tabular}

\section{D.1.4 SIMP continuation with threshold projection continuation}

The bridge example is also solved by considering the parallel continuation of penalization parameter $z$ and threshold projection parameter $\beta$. The results are presented in Figure D.5 and Table D.3. Each example of Figure D.5 uses different values for $z_{\min }$ and $z_{\max }$, but they all use $\beta_{\min }=1$ and $\beta_{\max }=8$. 


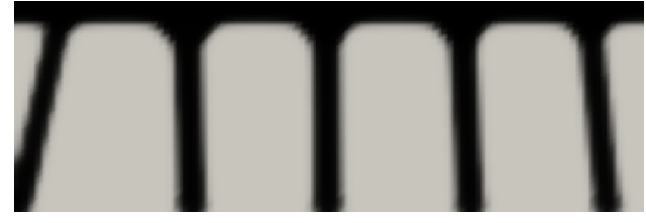

(a) $z_{\min }=1, z_{\max }=4$

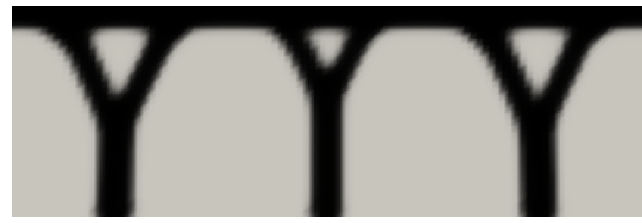

(c) $z_{\min }=3, z_{\max }=6$

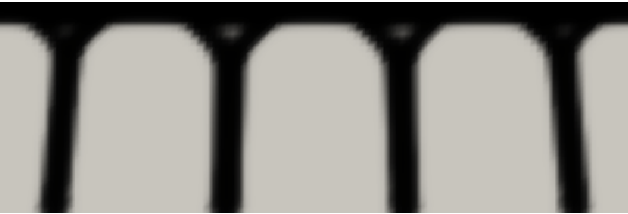

(b) $z_{\min }=2, z_{\max }=5$

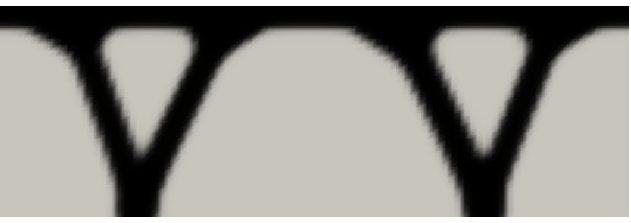

(d) $z_{\min }=4, z_{\max }=7$

Figure D.5 - Bridge topologies by considering continuation of penalization parameter $z$ (see Table D.3).

Table D.3 - Bridge results by considering continuation of penalization parameter $z$.

\begin{tabular}{ccc}
\hline Reference & $J_{C}$ & $M_{n d}(\%)$ \\
\hline Figure D.6a & 2.77 & 12.0 \\
Figure D.6b & 3.00 & 10.1 \\
Figure D.6c & 3.34 & 9.8 \\
Figure D.6d & 4.30 & 8.6 \\
\hline
\end{tabular}

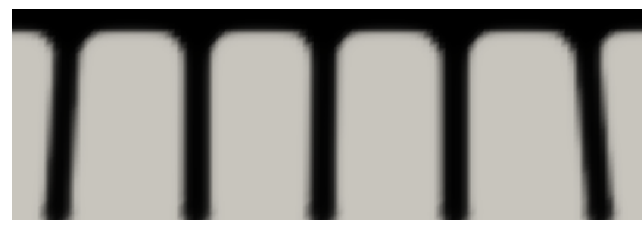

(a) $z_{\min }=1, z_{\max }=4$

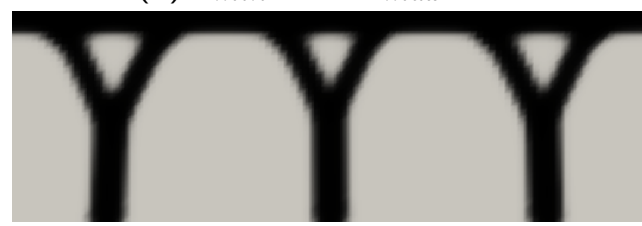

(c) $z_{\min }=3, z_{\max }=6$

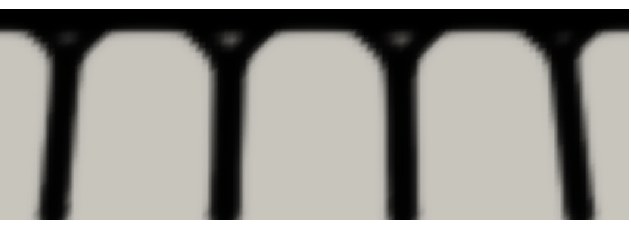

(b) $z_{\min }=2, z_{\max }=5$

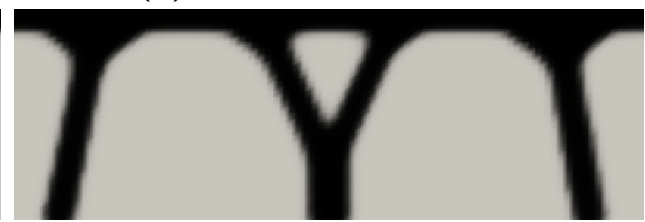

(d) $z_{\min }=4, z_{\max }=7$

Figure D. 6 - Bridge topologies by considering nearly incompressible material $\nu_{s}=0.49$ (see Table D.4).

\section{D.1.5 Nearly incompressibility}

The bridge example is also solved by considering nearly incompressibility $\nu_{s}=0.49$ and the results are presented in Figure D.6 and Table D.4. The threshold projection continuation goes from $\beta_{\min }=1$ to $\beta_{\max }=8$ for all examples and the continuation of SIMP penalization parameter $z$ is described at each result. 
Table D.4 - Bridge results by considering nearly incompressible material $\nu_{s}=0.49$.

\begin{tabular}{ccccc}
\hline Reference & $z_{\min }$ & $z_{\max }$ & $J_{C}$ & $M_{n d}(\%)$ \\
\hline Figure D.6a & 1 & 4 & 2.73 & 11.7 \\
Figure D.6b & 2 & 5 & 2.99 & 10.1 \\
Figure D.6c & 3 & 6 & 3.31 & 10.1 \\
Figure D.6d & 4 & 7 & 3.76 & 9.7 \\
\hline
\end{tabular}

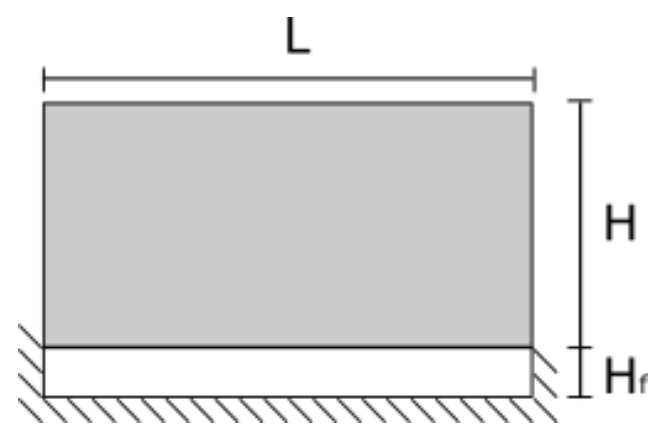

Figure D.7 - Design domain of internally pressurized lid example.

\section{D.2 Internally pressurized lid}

The internally pressurized lid is a benchmark problem used in several works of topology optimization involving design-dependent loads (HAMMER; OLHOFF, 2000; CHEN; KIKUCHI, 2001; DU; OLHOFF, 2004a; SIGMUND; CLAUSEN, 2007). Therefore, it is a suitable example to verify the implementation of the topology optimization code developed in this work as well as good source for studies.

This example consists of a structure whose pressure load is applied by an internally pressurized fluid. The design domain is a rectangle of length $L$ and height $H$. A narrow fixed fluid region of height $H_{f}$ is added to the bottom of the design domain according to Figure D.7. The dimensions used are presented in Equation D.9.

$$
\begin{aligned}
& L=1 \\
& H=0.5 \\
& H_{f}=0.1
\end{aligned}
$$

The design domain is discretized by FEniCS' right triangular elements and the element size is 0.02. Triangular Lagrange elements of degree two, one and one are used for displacement, pressure and design variable fields, respectively. Thus, the mesh has 2750 finite elements and the optimization problem has 1326 design variables.

The traction forces $\boldsymbol{t}$ are parameterized in terms of the pressure magnitude $p$ according to 
Equation D.10. A unit value for $p$ is considered if not otherwise noted.

$$
\boldsymbol{t}=\left[\begin{array}{l}
0 \\
p
\end{array}\right]
$$

The allowed material volume $V^{*}$ used in internally pressurized lid example is set to $50 \%$. By considering a unit depth, the value of $V^{*}$ is given by Equation D.11.

$$
V^{*}=0.5 L H
$$

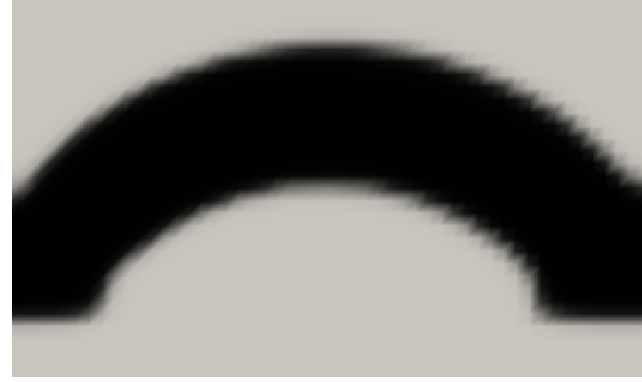

(a) Topology for $\nu_{s}=0.4$

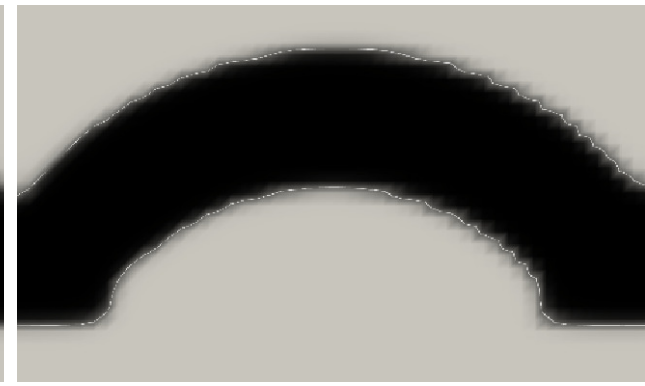

(b) Topology for $\nu_{s}=0.49$

Figure D.8 - Results for internally pressurized lid example by considering different $\nu_{s}$.

\section{D.2.1 Fluid compressibility}

The effect of fluid compressibility in optimization results can be checked by comparing results obtained with different levels of $K_{f}$.

Starting the analysis by increasing values of $K_{f}$, the results for $K_{f}=10^{3}, K_{f}=10^{4}, K_{f}$ $=10^{5}$, and $K_{f}=10^{6}$ are equivalents in terms of topology to Figure D.8a $\left(K_{f}=100\right)$, so they are not reproduced here.

For lower values of $K_{f}$, there are topology differences in relation to Figure D.8a as can be seen in Figure D.9. As the fluid behavior approaches air (void), the lower part of design domain closes.

Therefore, the fluid compressibility influences the result of the optimization, i.e. the optimized body depends if the fluid is an incompressible hydrostatic fluid, a compressible hydrostatic fluid, or air (void).

\section{D.2.2 Pressure load magnitude}

The influence of pressure load magnitude is assessed by running optimizations with different values of $p$. 


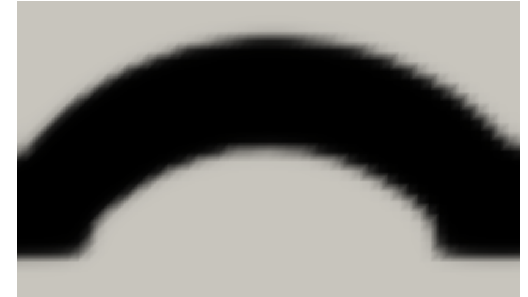

(a) $K_{f}=10$

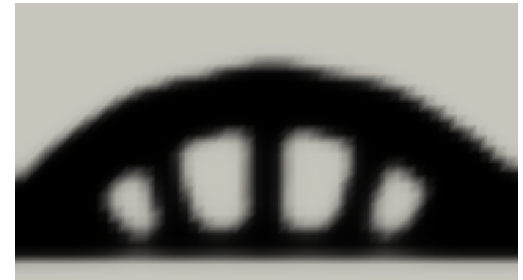

(d) $K_{f}=0.01$

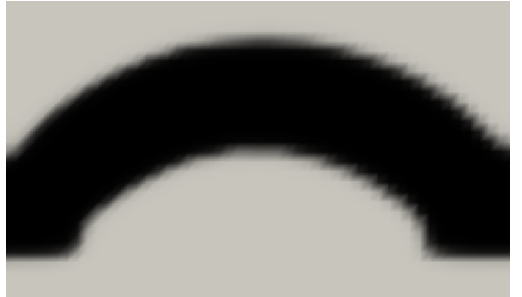

(b) $K_{f}=1$

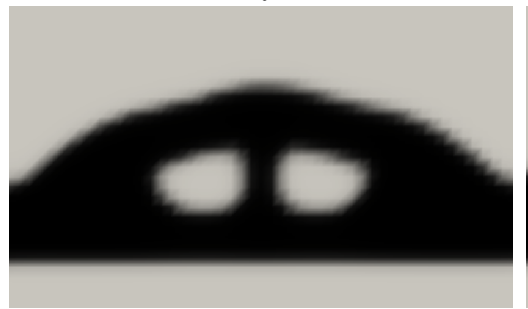

(e) $K_{f}=0.001$

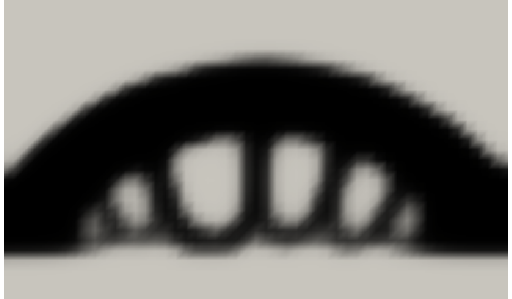

(c) $K_{f}=0.1$

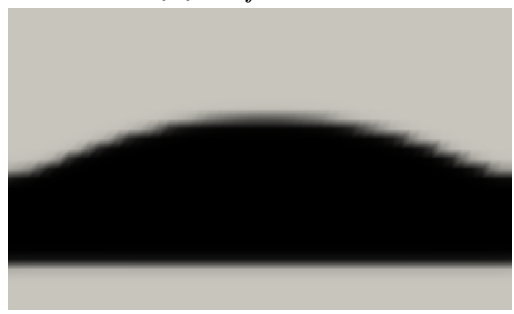

(f) $K_{f}=0.0001$

Figure D.9 - Results for internally pressurized lid example by considering different $K_{f}$.

There are no topological differences in results when the optimization is executed with higher values of $p$. The results are similar to Figure D.8a.

Low values of pressure load can compromise convergence according to D.10. This happens because the magnitude of the objective function becomes low for the tolerance being used. This is proved in Figure D.11 by reducing the $\epsilon_{t o l}$ and verifying the results. It is possible to see that the convergence is recovered when the tolerance is lower (Figure D.11c).

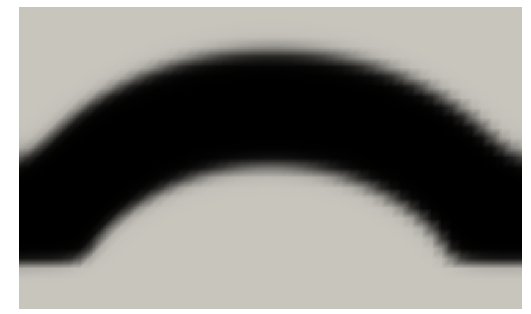

(a) $p=0.1$

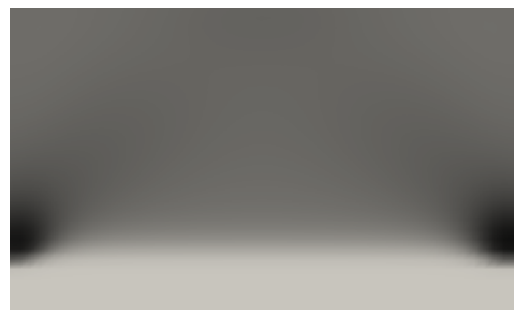

(b) $p=0.01$

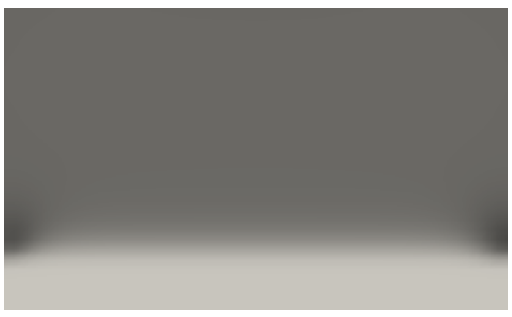

(c) $p=0.001$

Figure D.10 - Results for internally pressurized lid example by considering lower values of $p$.

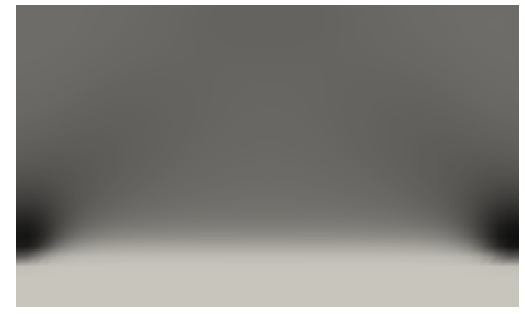

(a) $\epsilon_{\text {tol }}=10^{-3}$

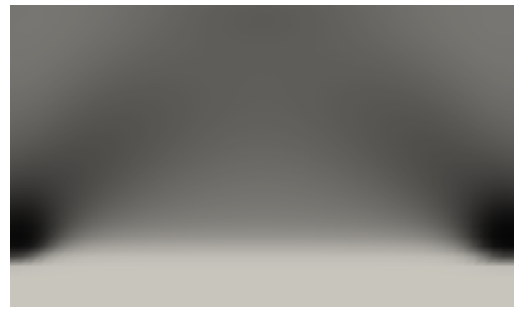

(b) $\epsilon_{t o l}=10^{-4}$

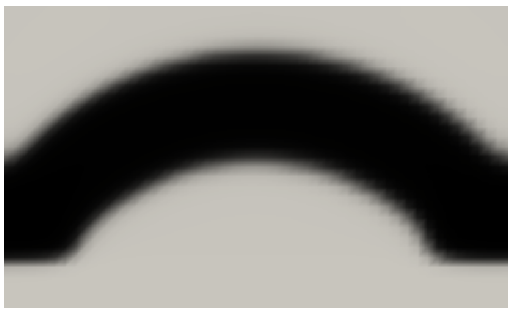

(c) $\epsilon_{\text {tol }}=10^{-5}$

Figure D.11 - Results for internally pressurized lid example by considering $p=0.01$ and different values of $\epsilon_{t o l}$. 


\section{D.3 Air Bulk Modulus}

This section considers the concept of an ideal gas to derive the bulk modulus of air during an isothermical process (BORGNAKKE; SONNTAG, 2009). For an ideal gas, the relation between pressure $p$, volume $V$ and temperature $T$ is given by Equation D.12 in which $R$ is the ideal gas constant.

$$
p=\frac{R T}{V}
$$

To compute the bulk modulus by using Equation A.1, it is necessary to differentiate the pressure with respect to the volume. If the temperature is constant, the derivative $\frac{d p}{d V}$ is given by Equation D.13.

$$
\frac{d p}{d V}=-\frac{R T}{V^{2}}
$$

Therefore, the bulk modulus for an ideal gas during an isothermical process is equal to the pressure according to Equation D.14.

$$
K=V \frac{R T}{V^{2}}=\frac{R T}{V}=p
$$

By considering air as an ideal gas at atmospheric pressure, the bulk modulus is given by Equation D.15.

$$
K=101 \mathrm{kPa}
$$

\section{D.4 Complete Code of Inverter Actuator Design}

Source Code D.1 - Complete code of inverter actuator design.

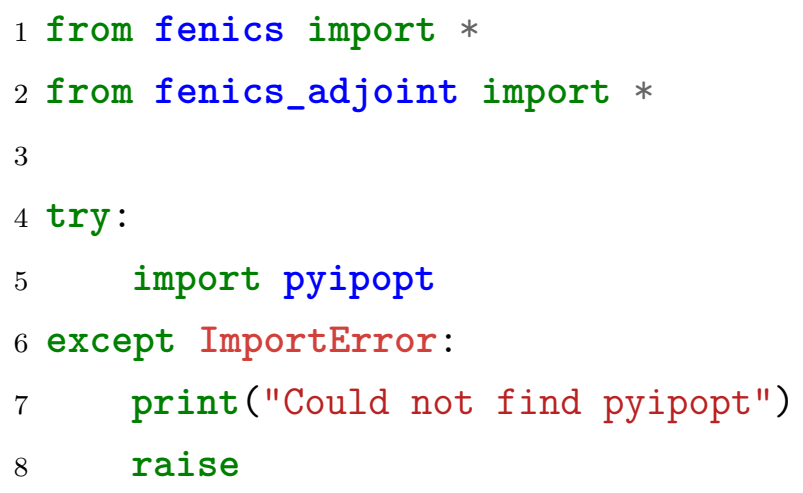




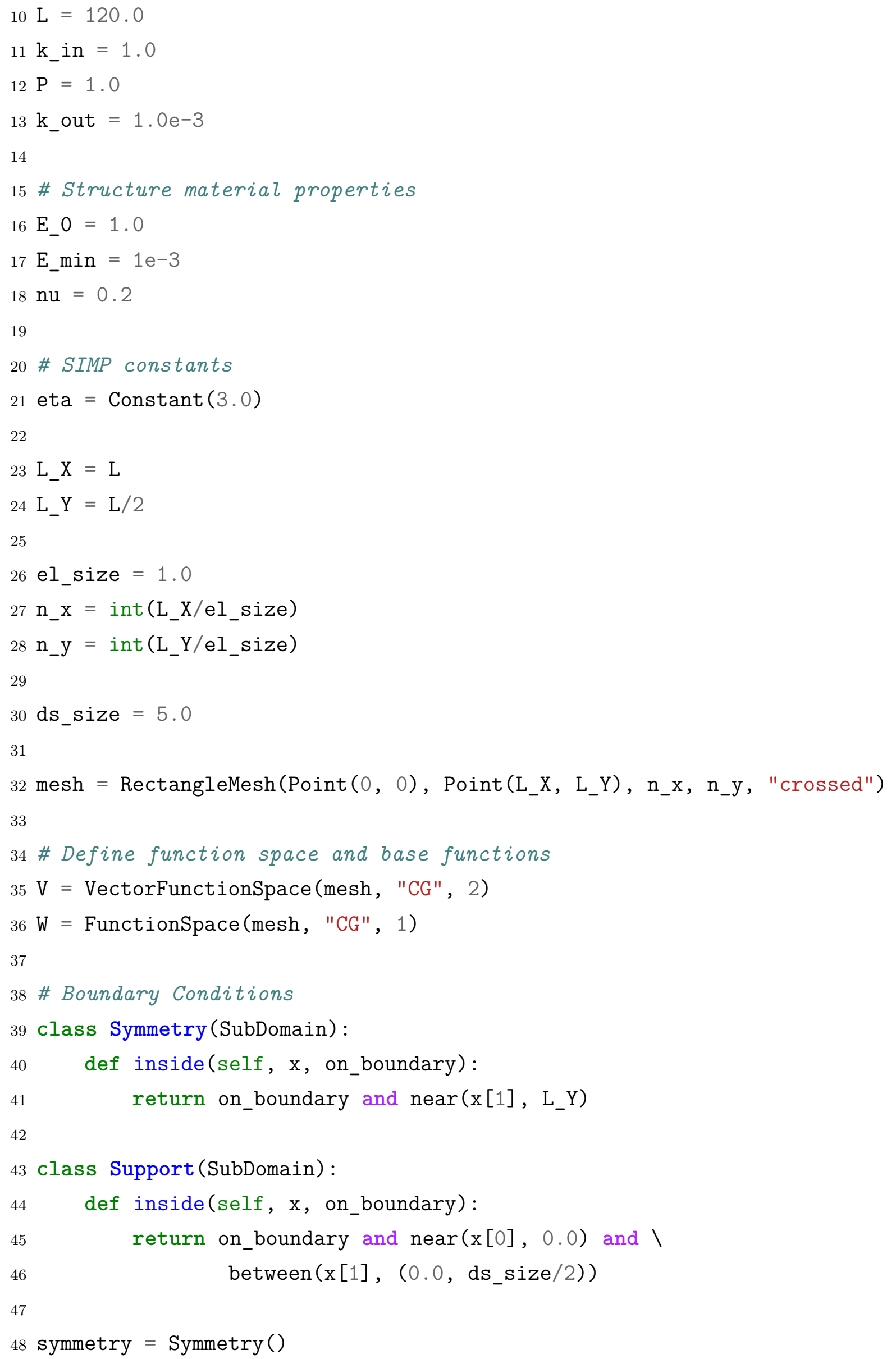




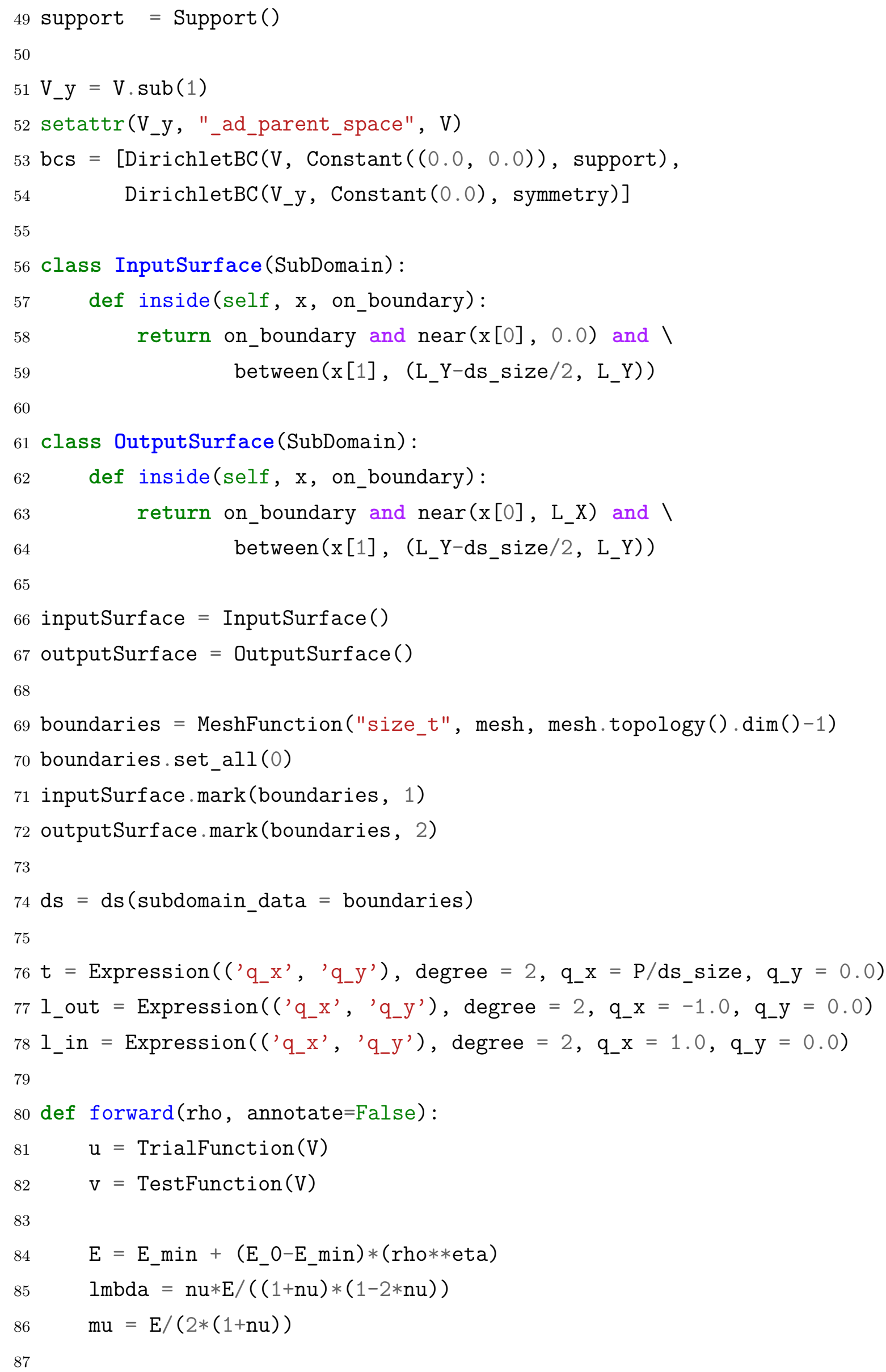


124 if

$$
\begin{aligned}
& \mathrm{a}=2 * \operatorname{mu} * \text { inner }(\operatorname{sym}(\text { nabla_grad }(u)), \quad \operatorname{sym}(\text { nabla_grad(v)) }) * d x \backslash \\
& +\operatorname{lmbda} * \operatorname{div}(\mathrm{u}) * \operatorname{div}(\mathrm{v}) * \operatorname{dx} \backslash \\
& +\left(\mathrm{k}_{-} \text {in/ds_size }\right) * i n n e r\left(\mathrm{u}, \mathrm{l}_{-} \text {in }\right) * \operatorname{inner}\left(\mathrm{v}, \mathrm{l}_{-} \text {in }\right) * \mathrm{ds}(1) \backslash \\
& +\left(\mathrm{k}_{-} \text {out } / \mathrm{ds}_{-} \text {size }\right) * \operatorname{inner}\left(\mathrm{u}, \text { I_out }_{-} * \operatorname{inner}\left(\mathrm{v}, \mathrm{I}_{-} \text {out }\right) * \mathrm{ds}(2)\right.
\end{aligned}
$$$$
\mathrm{L}=\operatorname{inner}(\mathrm{t}, \mathrm{v}) * \mathrm{ds}(1)
$$

$\mathrm{A}, \mathrm{b}=$ assemble_system ( $\mathrm{a}, \mathrm{L}, \mathrm{bcs})$

$\mathrm{u}=$ Function $(\mathrm{V}$, name="Displacement")

solve (A, u.vector ()$, b$, annotate=annotate $)$

return u

class VolumeConstraint(InequalityConstraint):

def__init_(self, V, W_sp):

self. $\mathrm{V}=\mathrm{V}$

self.smass $=$ assemble(TestFunction(W_sp) $*$ Constant $(1) * d x)$

self.tmpvec $=$ Function $\left(W_{-}\right.$sp)

def function(self, m):

from pyadjoint.reduced_functional_numpy import set_local set_local (self.tmpvec, m)

integral $=$ self. smass.inner $($ self $\cdot$ tmpvec $\cdot \operatorname{vector}())$

if MPI.rank(mpi_comm_world ()$)==0$ :

print("Current control integral: ", integral)

$\mathrm{g}=$ self. $\mathrm{V}$ - integral

return $[\mathrm{g}]$

def jacobian(self, m):

return [-self.smass]

def output_workspace(self):

return [0.0]

def length(self):

return 1

$f_{--}$name_--= "_main_-_"

rho = interpolate (Constant (0.5), W $)$

$\mathrm{u}=$ forward (rho, annotate=True) 
127

$128 \quad \mathrm{~J}=\operatorname{assemble}\left(-\operatorname{inner}\left(1_{\text {_out }}, \mathrm{u}\right) * \mathrm{ds}(2)\right)$

129 Jhat $=$ ReducedFunctional $(\mathrm{J}, \operatorname{Control}($ rho $))$

130

$131 \quad 1 b=0.0$

$132 \mathrm{ub}=1.0$

133 Vol_const $=$ VolumeConstraint $\left(0.25 * L_{-} \mathrm{X} * \mathrm{~L}_{-} \mathrm{Y}, \mathrm{W}\right)$

134 